\title{
LES CAHIERS
}

\section{DE RECHERCHES CRIMINOLOGIQUES}

$\mathbf{N}^{\circ} \mathbf{3 1}$

\section{LA COUVERTURE JOURNALISTIQUE DES REQUÊTES DE RÉVISION JUDICIAIRE AU CANADA}

\author{
Karl Thomassin \\ Pierre Landreville \\ (1999)
}

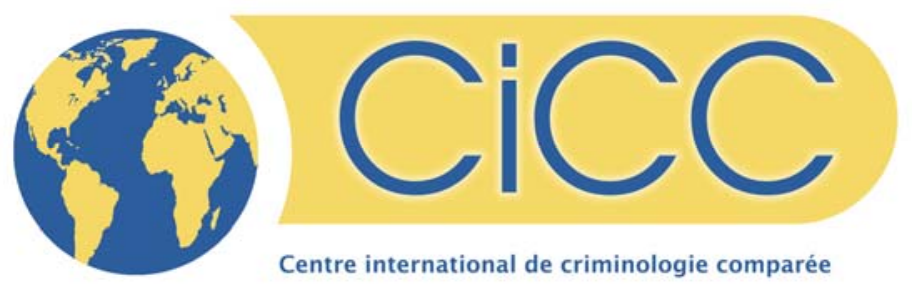

Université de Montréal

Case postale 6128, Succursale Centre-ville Montréal, Québec, H3C 3J7, Canada Tél.: 514-343-7065 / Fax.: 514-343-2269 cicc@umontreal.ca / www.cicc.umontreal.ca 


\title{
LA COUVERTURE JOURNALISTIQUE DES REQUÊTES \\ DE RÉVISION JUDICIAIRE AU CANADA
}

PAR

KARL THOMASSIN

sous la direction de

PIERRE LANDREVILLE

\author{
École de criminologie \\ Centre international de criminologie comparée \\ Université de Montréal \\ GROUPE DE RECHERCHE ET D'ANALYSE \\ SUR LES POLITIQUES ET LES PRATIQUES PÉNALES
}

1999

GRAPP

Centre international de criminologie comparée Département de sociologie Université de Montréal

Université du Québec à Montréal

Cette recherche a été réalisée grâce à des subventions du Conseil de recherche en sciences humaines du Canada et du Fonds pour la formation de chercheurs et l'aide à la recherche (FCAR soutien aux équipes). 


\section{SOMMAIRE}

La présente recherche porte sur la couverture journalistique des requêtes de révision judiciaire ayant eu lieu au Canada de 1987 au 31 mai 1998. La révision judiciaire (art.745.6 du C. cr.) est une disposition légale qui permet aux individus condamnés pour meurtre, et qui doivent purger plus de quinze années d'emprisonnement, de demander une révision judiciaire de leur période d'inadmissibilité à la libération conditionnelle.

La recherche vise à compléter quatre objectifs, soit: évaluer l'étendue de la couverture journalistique des requêtes de révision judiciaire, analyser le contenu de cette couverture, identifier les facteurs associés à l'intensité de la couverture et finalement, analyser la relation entre la couverture journalistique des requêtes et la décision des jurys de réduire le délai d'inadmissibilité à la libération conditionnelle.

Afin de réaliser ces objectifs, deux sources de données furent mises à contribution. Tout d'abord, une liste incluant l'ensemble des requêtes de révision judiciaire, fournie par le Service Correctionnel du Canada (SCC), contenait plusieurs informations-clés à leur sujet. Avec les informations concernant le lieu et le moment où ces 92 requêtes se sont déroulées, un ensemble de quotidiens canadiens fut consulté. Suite à cette consultation, un nombre important d'articles $(n=365)$ a été échantillonné et analysé en conséquence.

Les résultats démontrent que l'étendue et le contenu des articles varient grandement selon les différentes régions du Canada. À l'Ouest, les requêtes font l'objet d'une couverture journalistique beaucoup plus intense qu'au Québec. D’autre part, les articles des quotidiens de l'Ouest sont plus souvent défavorables au requérant et/ou à la réduction du délai d’inadmissibilité à la libération conditionnelle. Puis, contrairement à ce qui était mentionné dans la littérature, très peu d'articles ont expliqué le processus de révision judiciaire de façon inexacte. D’autre part, le fait que le requérant ait tué un 
policier, ainsi que le fait que des opposants à la révision judiciaire (groupes de pression, policiers, et politiciens) soient présents lors du déroulement de la requête, sont associés avec l'intensité de publication des requêtes. Finalement, la décision du jury de réduire le délai d'inadmissibilité à la libération conditionnelle du requérant est associée avec le fait que la requête ait paru ou non dans les quotidiens échantillonnés. 


\section{TABLE DES MATIÈRES}

SOMMAIRE.

TABLE DES MATIÈRES . $\mathbf{v}$

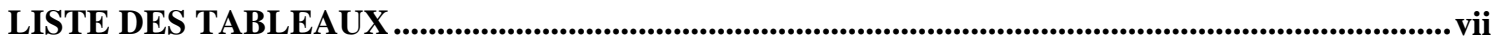

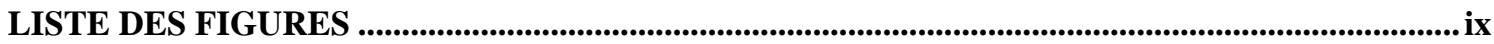

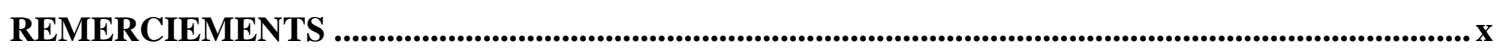

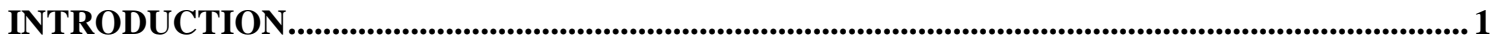

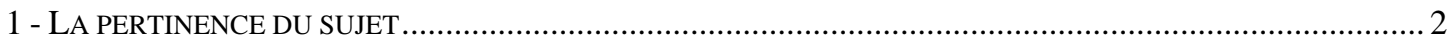

2 - LES OBJECTIFS ET LA MÉTHODOLOGIE DE LA RECHERCHE .................................................................... 3

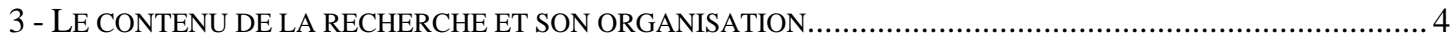

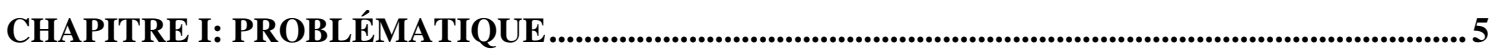

1.1 - LA COUVERTURE JOURNALISTIQUE DES TRIBUNAUX DE JURIDICTION CRIMINELLE ............................. 6

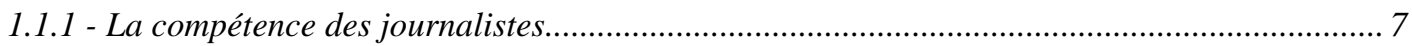

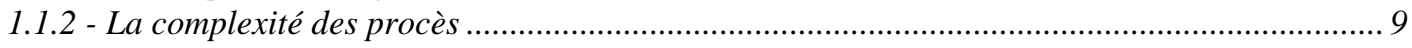

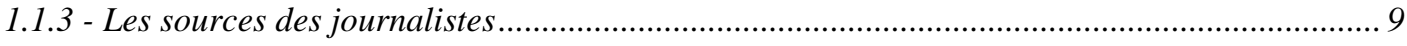

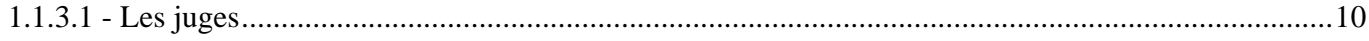

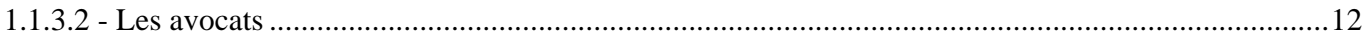

1.1.3.3 - Les commis des palais de justice ....................................................................................................14

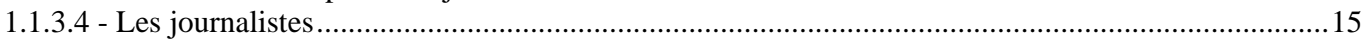

1.1.3.5 - Les documents ...................................................................................................................16

1.1.4 - Les contraintes organisationnelles des maisons de presse et des tribunaux............................ 17

1.1.4.1 - La contrainte temporelle...............................................................................................................

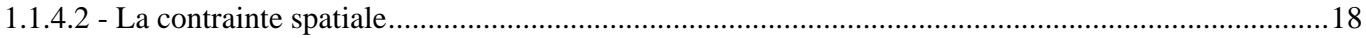

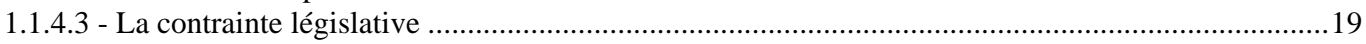

1.1.4.4 - Le critère de "newsworthiness" ........................................................................................................2

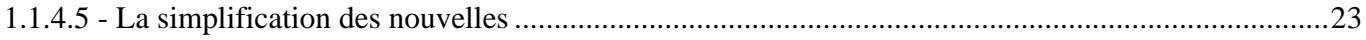

1.1.5 - La corrélation entre la couverture journalistique des procès en droit criminel et la décision

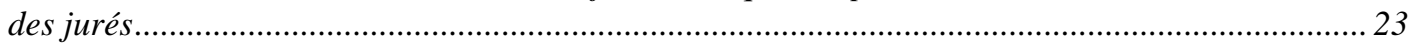

1.2 - LA RÉVISION JUDICIAIRE DE LA PÉRIODE D'INADMISSIBILITÉ À LA LIBÉRATION CONDITIONNELLE ... 24

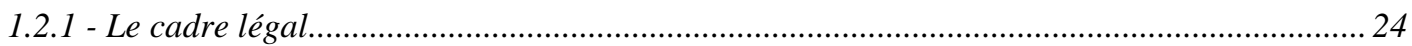

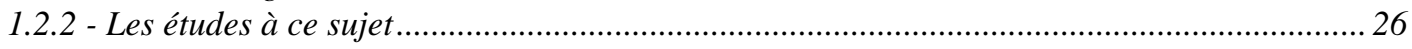

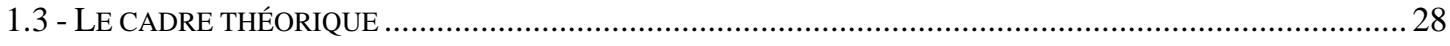

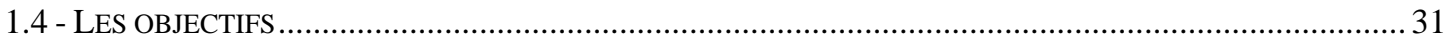

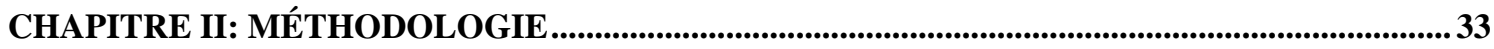

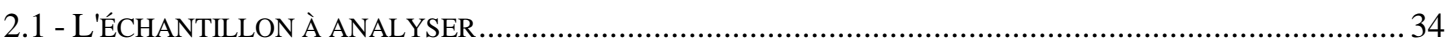

2.1.1 - Critères d'échantillonnage des articles de quotidiens canadiens ............................................... 35

2.1.1.1 - Échantillonner le(s) quotidien(s) de la province dans laquelle une ou plusieurs requêtes de révision judiciaire ont eu lieu (sauf pour le quotidien Globe and Mail) ......................................................................35

2.1.1.2 - Échantillonner le(s) quotidien(s) le(s) plus lu(s) dans la province..................................................35

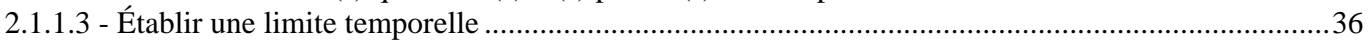

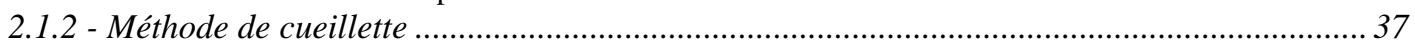

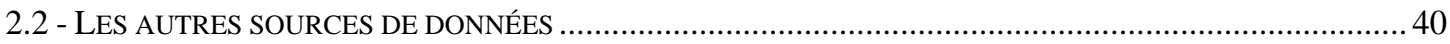

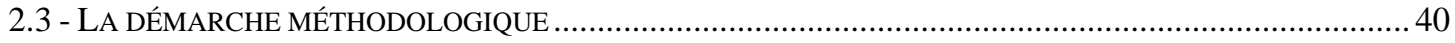

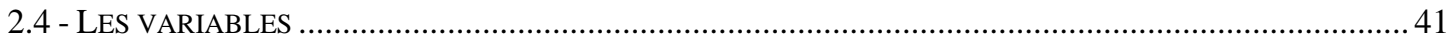

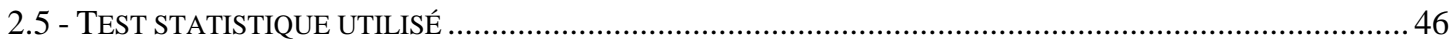




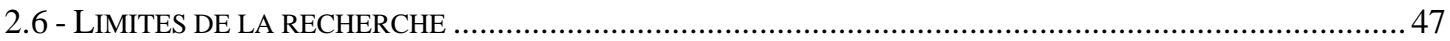

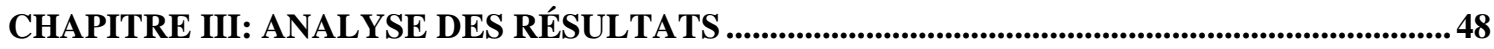

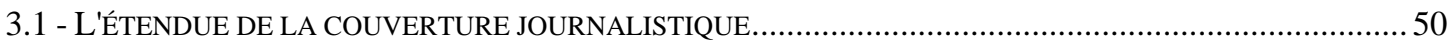

3.1.1 - L'étendue de la couverture journalistique selon les régions du Canada .................................50

3.1.2 - L'étendue de la couverture journalistique selon les années...................................................... 54

3.1.3 - L'étendue de la couverture journalistique selon les types de quotidien ...................................56

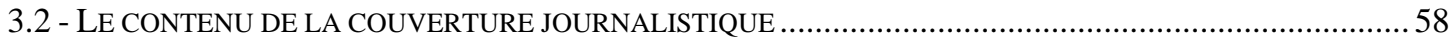

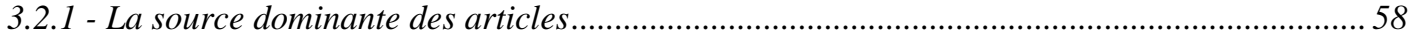

3.2.1.1 - La source dominante des articles selon les régions du Canada.........................................................60

3.2.1.2 - La source dominante des articles selon les types de quotidien .........................................................61

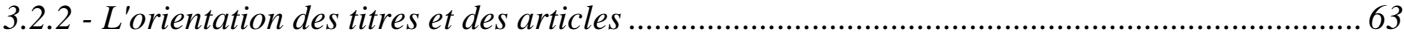

3.2.2.1 - L'orientation des titres et des articles selon les régions du Canada....................................................63

3.2.2.2 - L'orientation des récits secondaires .......................................................................................68

3.2.2.3 - L'orientation des titres et des articles selon les types de quotidien .................................................70

3.2.2.4 - L'orientation des titres et des articles selon les caractéristiques liées aux homicides commis par les

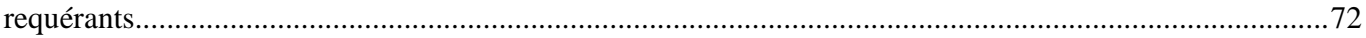

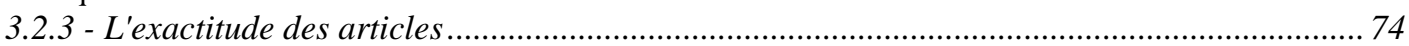

3.2.3.1 - L'exactitude des articles selon les régions du Canada .................................................................75

3.2.3.2 - L'exactitude des articles selon les types de quotidien....................................................................76

3.3 - LES FACTEURS ASSOCIÉS À L'INTENSITÉ DE LA COUVERTURE JOURNALISTIQUE DES REQUÊTES ........ 77

3.4 - LES FACTEURS ASSOCIÉS À LA RÉDUCTION ÉVENTUELLE DU DÉLAI D'INADMISSIBILITÉ À LA

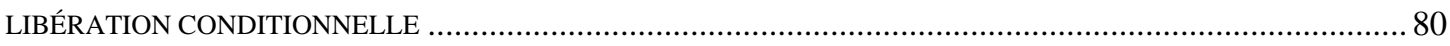

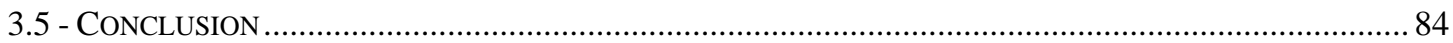

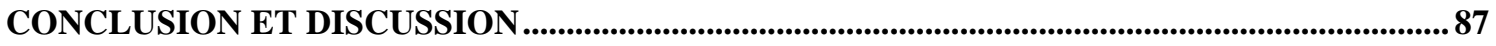

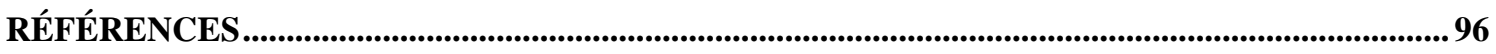

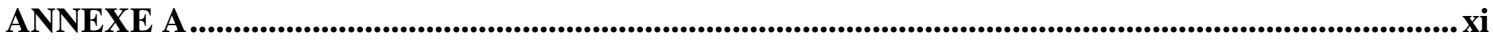

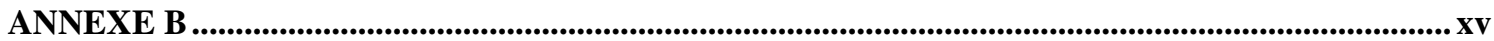




\section{LISTE DES TABLEAUX}

Tableau I: Nombre d'articles échantillonés selon les quotidiens des différentes provinces

Tableau II: Variables des requêtes de révision judiciaire

Tableau III: Variables des articles de quotidiens canadiens traitant des requêtes de révision judiciaire.

Tableau IV: Type de récit et redondance des articles selon les provinces du Canada.....45

Tableau V: Publication journalistique des requêtes de révision judiciaire selon les régions du Canada

Tableau VI: Requêtes de révision judiciaire au Québec selon les palais de Justice. .51

Tableau VII: Ordre chronologique des requêtes publiées selon les régions de l'Ontario et du Québec

Tableau VIII: Nombre d'articles consacrés à chaque requête publiée selon les régions du Canada

Tableau IX: Pourcentage de requêtes publiées et nombre d'articles consacrés à chaque requête publiée selon les types de quotidiens de la Colombie-Britannique et du Québec

Tableau X: Source dominante de l'article .59

Tableau XI: Source dominante de l'article selon les régions du Canada

Tableau XII: Source dominante de l'article selon les types de quotidien de la ColombieBritannique et du Québec.

Tableau XIII: Orientation du titre et de l'article selon les régions du Canada.

Tableau XIV: Orientation de l'article selon les types de récit secondaire

Tableau XV: Orientation du titre et de l'article selon les types de quotidien de la Colombie-Britannique et du Québec.

Tableau XVI: Orientation du titre et de l'article selon les caractéristiques liées aux homicides 
Tableau XVII: Exactitude de l'article selon les régions du Canada. .75

Tableau XVIII: Exactitude de l'article selon les types de quotidien de la Colombie-

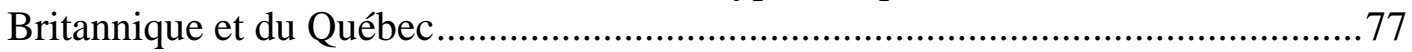

Tableau XIX: Nombre d'articles consacrés aux requêtes selon leurs caractéristiques ....79

Tableau XX: Présence d'opposants lors de la requête selon les types de victime

Tableau XXI: Décision au sujet de la période d'inadmissibilité à la libération conditionnelle selon les caractéristiques liées aux requêtes

Tableau B1: Exactitude de l'article selon le type de récit secondaire .............................xvi

Tableau B2: Exactitude de l'article selon les caractéristiques liées aux homicides........xvi 


\section{LISTE DES FIGURES}

Figure 1: Répartition des articles échantillonnés $(n=365)$ selon les jours précédant ou suivant la décision du jury

Figure 2: Requêtes et requêtes publiées selon les années.

Figure 3: Requêtes et articles publiés selon les années ....................................................................................56 


\section{REMERCIEMENTS}

La présente recherche a pu être réalisée en partie grâce au programme de bourses pour les études de cycles supérieurs en recherche du Fonds pour la formation de chercheurs et l'aide à la recherche. 
INTRODUCTION 


\section{1 - La pertinence du sujet}

Lors de l'abolition de la peine de mort au Canada, en 1976, une nouvelle disposition légale fut introduite dans le Code criminel: il s'agit de la révision judiciaire (art. 745.6 du C.cr.). Celle-ci permet aux individus condamnés pour meurtre, et qui doivent purger plus de quinze années d'emprisonnement, de demander une révision judiciaire de leur période d'inadmissibilité à la libération conditionnelle. Un jury doit alors déterminer s'il y a lieu de réduire le délai préalable à la libération conditionnelle du requérant. La première requête de révision judiciaire a eu lieu en 1987 et depuis ce temps, un nombre croissant de requêtes se déroulent chaque année (O'Reilly-Fleming, 1991; Roberts, 1994a; Pilon, 1993/1997).

Toutefois, le déroulement de ces requêtes ne se fait pas sans bruit. Comme Roberts (1994b) le rapporte:

"In light of the growing number of applications for judicial review..., this issue will receive an even higher profile in coming years. Those applications that have been reviewed have already generated a great deal of adverse publicity and public hostility towards the criminal justice system." (40)

Les opposants à la révision judiciaire considèrent qu'elle est une échappatoire antidémocratique à la loi, et ils alimentent la peur du public en tentant de leur faire croire que de dangereux meurtriers seront remis en liberté grâce à cette disposition

légale (Roberts, 1995). Ces propos se reflètent notamment dans les médias, où la révision judiciaire fait l'objet d'une couverture négative abondante (Roberts, 1997). Cependant, aucune étude ne s'est attardée à analyser systématiquement la couverture médiatique rattachée à la révision judiciaire. Le peu de chercheurs ayant abordé la question se sont limités à quelques commentaires généraux ou à l'énumération de faits anecdotiques ayant trait à ce sujet. Une analyse systématique de cette couverture s'avère pourtant nécessaire afin de mieux cerner la façon dont les médias traitent des requêtes de 
révision judiciaire à travers le Canada. Ainsi, il serait possible de comparer la réalité des requêtes telle que retrouvée dans les statistiques judiciaires, avec leur réalité telle que présentée dans les médias.

\section{2 - Les objectifs et la méthodologie de la recherche}

La présente recherche vise donc à analyser la couverture médiatique réservée aux requêtes de révision judiciaire à partir d'un des médias de masse, soit la presse. Concrètement, quatre objectifs de recherche ont été formulés, soit:

- Évaluer l'étendue de la couverture journalistique des requêtes de révision judiciaire.

- $\quad$ Analyser le contenu rapporté dans la couverture journalistique des requêtes de révision judiciaire.

- $\quad$ Identifier les facteurs associés à l'intensité de la couverture journalistique des requêtes de révision judiciaire.

- $\quad$ Analyser la relation entre la couverture journalistique des requêtes de révision judiciaire et la décision des jurys de réduire le délai d'inadmissibilité à la libération conditionnelle.

Pour réaliser ces objectifs, une liste des requêtes de révision judiciaire fournie par le Service Correctionnel du Canada a d'abord été mise à contribution. Cette liste, qui comprenait plusieurs informations-clés au sujet de chacune des requêtes s'étant déroulées au Canada entre 1987 (l'année où la première requête a eu lieu) et le 31 mai 1998 ( $\mathrm{n}=92)$, a servi de base pour le processus d'échantillonnage. Avec certaines de ces informations-clés en main, effectivement, il a été possible d'aller échantillonner 
plusieurs quotidiens canadiens pour colliger les articles traitant des requêtes de révision judiciaire $(n=365)$.

\section{3 - Le contenu de la recherche et son organisation}

Dans le premier chapitre de la recherche, l'état des connaissances au sujet de la couverture journalistique des tribunaux de juridiction criminelle est exposé. Nous n'avons pu nous limiter aux études concernant uniquement la couverture journalistique des requêtes de révision judiciaire car elles ne s'avéraient pas assez nombreuses. Par la suite, le cadre légal de la révision judiciaire est brièvement explicité, suivi du cadre théorique de la recherche. Finalement, les objectifs de la recherche sont énumérés.

Le second chapitre traite des sources de données et de la méthodologie utilisées afin de mener à bien la recherche. D'autre part, les variables construites à partir des sources de données sont énumérées et explicitées. La nature du test statistique utilisé dans la recherche est également expliquée. Finalement, les limites rattachées aux sources de données et à la méthodologie sont mentionnées.

Au troisième chapitre, l'analyse des données est effectuée. Ainsi, nous constatons notamment que l'étendue de la couverture journalistique des requêtes varie selon les régions du Canada. Il en va de même pour le contenu de cette couverture journalistique: la source dominante, l'orientation et l'exactitude des articles varient effectivement selon les différentes régions.

En conclusion, nous présentons les points saillants de la recherche, ainsi que les implications générales pouvant leur être rattachées. Puis, les limites de la recherche sont évoquées afin de souligner leur impact possible sur les résultats de la recherche. Finalement, de nouvelles questions sont soulevées pour favoriser de nouvelles pistes de recherche au sujet de la couverture journalistique des requêtes de révision judiciaire. 


\section{CHAPITRE I}

PROBLÉMATIQUE 
Peu de recherches ont abordé la couverture journalistique des requêtes de révision judiciaire. Jusqu'à maintenant, elles se limitent à des commentaires généraux sur ce que les quotidiens rapportent à propos de cette disposition législative, aucune d'elles n'ayant effectué une analyse systématique à ce sujet. Or, une telle démarche s'avère nécessaire afin de vérifier la validité de ces commentaires. La présente recherche aura donc pour but d'analyser le contenu des quotidiens canadiens au sujet des requêtes de révision judiciaire. De plus, le contenu des journaux sera comparé à un contenu provenant des institutions de justice criminelle afin de souligner les différences ou les ressemblances existant entre les deux réalités présentées.

Comme c'est le cas pour les études traitant de la presse et de la révision judiciaire, les ouvrages au sujet de la couverture journalistique des tribunaux de juridiction criminelle ne sont pas nombreux. Malgré tout, une revue de littérature à ce sujet permettra d'établir le cadre général de la recherche et ce, étant donné que les requêtes de révision judiciaire sont entendues devant les tribunaux de juridiction criminelle. Ce n'est que par la suite que nous aborderons les études traitant spécifiquement de la couverture journalistique de la révision judiciaire, précédé d'un bref survol du cadre légal rattaché à cette disposition législative. Puis, nous présenterons le cadre théorique de la présente recherche et nous terminerons en formulant nos objectifs spécifiques.

\section{1 - La couverture journalistique des tribunaux de juridiction criminelle}

La majorité des études portant sur la couverture journalistique de la justice criminelle visent à évaluer la nature et la fréquence des crimes retrouvés dans les journaux (voir notamment Davis, 1952; Antunes et Hurley, 1977; Graber, 1979; Ditton et Duffy, 1983; Doob, 1985; Gabor et Weimann 1987; Schlesinger et al., 1991; Jerin et Fields, 1994; Chermak, 1998a). Ainsi, peu d'études ont abordé la couverture journalistique des tribunaux de juridiction criminelle (Drechsel, 1983; Ericson et al., 
1989; Lotz, 1991). Ce constat s'avère surprenant, car lorsque les journaux abordent un sujet touchant la justice criminelle, les tribunaux font souvent l'objet de cette couverture journalistique. Lors d'une analyse de contenu de quarante journaux d'Ontario qui parurent en décembre 1968, par exemple, Dussuyer (1979) a divisé les articles traitant de la justice criminelle selon trois phases: la phase policière (découverte du crime, enquête, arrestation et mise en accusation), la phase judiciaire (de l'enquête préliminaire au verdict et à la sentence, le cas échéant) et la phase correctionnelle (incarcération, probation et libération conditionnelle). Ses résultats ont démontré que la phase judiciaire faisait l'objet de $46.9 \%$ de la couverture journalistique de la justice criminelle, comparativement à $42.5 \%$ pour la phase policière et $4.3 \%$ pour la phase correctionnelle. De son côté, Graber (1980) a démontré que sur l'ensemble des articles traitant de la justice criminelle dans le Chicago Tribune, entre le 15 janvier 1976 et le 14 janvier 1977, 32\% d'entre eux avaient comme sujet principal les tribunaux et leurs procédures, contre seulement $10 \%$ pour les activités des corps policiers et $2 \%$ pour les systèmes correctionnels. Finalement, Chermak (1994) a examiné la répartition des articles de justice criminelle du Midwest Tribune (un nom fictif d'un journal américain) selon les étapes du processus judiciaire. Ses résultats révèlent que 43.5\% des articles référaient à la phase judiciaire (la mise en accusation étant incluse dans cette catégorie), alors que $38.3 \%$ et $4.2 \%$ traitaient respectivement de la phase policière et correctionnelle.

La place importante qu'occupent les articles de quotidien ayant trait aux tribunaux justifie donc le besoin de s'y attarder davantage. Suite à la lecture d'études qui ont porté sur ce sujet, cinq thèmes furent constitués afin de dresser un portrait de la couverture journalistique des tribunaux de juridiction criminelle.

\subsection{1 - La compétence des journalistes}

Les auteurs s'entendent unanimement sur un point: les reporters affectés aux tribunaux manquent d'expérience et n'ont pas une connaissance suffisante des 
procédures judiciaires (Drechsel, 1978, 1983; Ericson et al. 1989; Grabosky et Wilson, 1989; Lotz, 1991). Cette critique revient continuellement dans la littérature et ce, autant du point de vue des auteurs que des sources des journalistes (juges et avocats, notamment) ayant été interviewées. Plusieurs raisons justifient l'opinion des chercheurs et des sources. Premièrement, il arrive bien souvent que ces journalistes en soient à leur premier emploi en tant que reporter (Drechsel, 1978). D'après les dires des sources potentielles retrouvées dans les tribunaux (juges et avocats), les chroniqueurs judiciaires se situent au bas de la hiérarchie du journalisme. Ainsi, le domaine de la chronique judiciaire serait un lieu d'apprentissage où de jeunes reporters s'initieraient au métier de journaliste (Ericson et al., 1989). À ce sujet, Ericson et al. (1989) mentionnent des faits anecdotiques qui sont survenus lors de leur recherche ethnographique auprès de journalistes couvrant les tribunaux à Toronto. Entre autres, ces derniers demandaient souvent des éclaircissements aux chercheurs afin de mieux cerner les aspects légaux qui étaient en jeu lors de procès (!). Aussi, plusieurs sources (dont les juges) considèrent qu'une quantité importante de faits erronés se retrouvent dans les articles de journaux traitant des procès en droit criminel, et ils attribuent ce fait au manque de connaissances et d'expérience de la part des reporters (Ericson et al., 1989; Lotz, 1991).

Toutefois, Lotz (1991) nuance ses propos à ce sujet. En prenant comme contreexemple le New York Times, il avance que cette remarque ne s'applique pas à la totalité des maisons de presse. Ainsi, certaines de ces institutions confieraient la couverture journalistique des tribunaux criminels à des reporters expérimentés. Drechsel (1978) nuance également ses déclarations et ce, étant donné que les reporters qu'il a interviewés ne travaillaient que dans la région non-métropolitaine du Minnesota. Selon lui, les reporters des quotidiens situés dans les grandes villes pourraient être plus expérimentés que ceux retrouvés dans les plus petites régions. L'étude d'Ericson et al. (1989) semble toutefois contredire cette proposition car elle a été effectuée dans une grande ville (Toronto). En somme, donc, il semble bel et bien que les journalistes assignés à la 
couverture des tribunaux criminels manquent d'expérience, ce qui pourrait nuire à la qualité des chroniques judiciaires publiées dans les journaux.

\subsection{2 - La complexité des procès}

Lors du déroulement d'un procès, il devient difficile de comprendre l'ensemble des recours utilisés par les deux partis impliqués (la Couronne et la défense). Hormis les juges, seuls ces partis possèdent les connaissances nécessaires (formation universitaire, jurisprudence) pour apprécier le déroulement et le dénouement des procès qui ont lieu. Conséquemment, la couverture journalistique des procès peut s'avérer une tâche ardue et ce, étant donné le langage hermétique des juges et des avocats, et la complexité des procédures judiciaires qui peuvent se présenter. Sur cet aspect, Lotz (1991) rapporte: "a trial certainly is not as simple as, say, a fire or an accident. Court cases go through a series of stages, and they hinge on questionable facts and esoteric legal issues..." (121). D'autre part, il n'est pas surprenant qu'un reporter ne saisisse pas toutes les subtilités d'un procès, puisqu'il arrive parfois que les avocats se préparent plusieurs semaines avant que celui-ci ne débute (Ericson et al., 1989). Conséquemment, les journalistes doivent souvent s'appuyer sur les propos de leurs sources afin d'avoir une meilleure appréciation des enjeux retrouvés dans les procès. Voyons maintenant à qui ces journalistes s'adressent pour parvenir à cette fin.

\subsection{3 - Les sources des journalistes}

Afin de pouvoir rédiger leurs chroniques judiciaires, les journalistes doivent s'adresser à différentes sources. Il faut préciser à l'avance que les journalistes passent très peu de temps à observer le déroulement des procès dans les salles d'audience (Ericson et al., 1989; Lotz, 1991) et ce, étant donné les contraintes organisationnelles qui leur sont imposées. Ces contraintes organisationnelles seront explicitées dans un point

ultérieur. Pour l'instant, nous nous limiterons à dire que les contraintes imposées aux 
journalistes justifient l'utilisation de l'entrevue (plutôt que l'observation dans les salles d'audience) comme méthode de prédilection pour couvrir les événements qui se sont déroulés dans les tribunaux (Ericson et al., 1989; Lotz, 1991).

Contrairement au milieu politique, où les politiciens s'intéressent grandement à la couverture médiatique dont ils font l'objet (Kent, 1985), les sources des tribunaux ne cherchent pas à tâter le pouls de l'opinion publique au sujet des décisions qu'ils prennent (Drechsel, 1983). D'autre part, ces sources ne s'attirent pas de la publicité favorable (Drechsel, 1983; Lotz, 1991) comme le font les corps policiers (Chermak, 1998b; Parent, 1987). Ces faits affectent indéniablement les relations entre les sources des tribunaux et les reporters:

"Compared to their colleagues on the police and legislature beats, court reporters had a more distant relationship to sources, mainly because lawyers, Crown attorneys, and judges have less need to use the news media to serve the purposes of the court system or their personal causes." (Ericson et al., 1989:37)

Ces caractéristiques générales étant exposées, nous allons maintenant aborder les principales sources des reporters. De cette façon, il sera possible de connaître la nature des liens qui unissent les acteurs du champ judiciaire et les journalistes.

\subsubsection{1 - Les juges}

Les reporters s'adressent rarement aux juges des tribunaux criminels (Morissette, 1991; Stanga, 1971). Pour expliquer ce fait, Ericson et al. (1989) postulent que parmi les juges, une règle implicite stipulerait qu'ils ne peuvent s'adresser aux différents médias et ce, notamment pour éviter qu'ils évaluent à nouveau ("second-guess") les décisions qu'ils ont prises lors de procès. D’autre part, ces auteurs rapportent l'opinion des juges au sujet de la place que devrait occuper le chroniqueur judiciaire dans les tribunaux: selon ces derniers, il ne devrait s'affairer qu'à recueillir les informations dans 
la salle d'audience, et nulle part ailleurs. Toutefois, Drechsel (1978) présente des résultats contradictoires: après avoir distribué des questionnaires auprès de journalistes attachés à des quotidiens non-métropolitains du Minnesota, il en conclut que les juges constituent l'une des sources les plus utilisées par les reporters. Cette contradiction pourrait provenir de l'échantillonnage des villes examinées par chacun des auteurs. La recherche d'Ericson et al. (1989) se concentrait à Toronto, l'une des plus grandes villes du Canada, alors que la recherche de Drechsel (1978) se limitait aux régions nonmétropolitaines du Minnesota. Or, il se pourrait que les tribunaux situés dans les régions non-métropolitaines, où moins de juges siègent et où moins de reporters sont affectés pour couvrir les procès, favorisent le développement de contacts plus intimes et personnels entre ces deux types de protagonistes. La ville de Toronto, avec la multitude de reporters et de juges qu'elle englobe, ne saurait permettre l'établissement de tels liens.

Même si la quasi-totalité des études mentionnent que les juges sont rarement interviewés, il semble toutefois que la qualité des relations existant entre ceux-ci et les journalistes varie d'une étude à l'autre. Comme mentionné précédemment, les juges interrogés par Ericson et al. (1989) ne s'attardent pas à former des liens avec les chroniqueurs judiciaires. À l'autre extrémité, Stanga (1971) rapporte que la majorité des juges de trois villes du Wisconsin qu'il a interviewés, entretenaient de bonnes relations avec les reporters. Finalement, Morissette (1991) expose la position mitoyenne suivante et ce, à partir de son expérience en tant que chroniqueur judiciaire pour le Journal de Montréal:

"Les juges ont des attitudes variées quant à leurs rapports avec la presse. Certains refusent en tout temps de parler aux journalistes. D'autres s'en accomodent simplement ou se montrent très loquaces. Cela dit, on connaît (heureusement) peu de juges qui acceptent de commenter une affaire en cours." (102) 


\subsubsection{2 - Les avocats}

Selon Ericson et al. (1989), il semble que les procureurs de la Couronne soient la source de prédilection des journalistes afin qu'ils puissent rédiger leurs chroniques judiciaires:

"Crown attorneys were the source for information about case updates, issues, outcomes, and facts. There was a prevailing view among court reporters that if some detail was missed while listening to the court case, or the hearing was not attended at all, the Crown attorney could supply the information most readily and authoritatively." (71)

Étant donné qu'ils fréquentent régulièrement les mêmes palais de justice, les procureurs de la Couronne sont en mesure d'informer les reporters au sujet des procès qui devraient faire l'objet d'une couverture journalistique (Ericson et al., 1989). De plus, ils peuvent conseiller les journalistes sur la façon de présenter les procès dans les quotidiens, tout en leur indiquant les dates où d'importants témoins viendront faire des déclarations sous serment (Chermak, 1998b).

Les procureurs de la Couronne représentent donc une source avantageuse pour les journalistes, d'autant plus qu'ils discutent volontiers des cas qui leur sont assignés (Chermak 1998b). À ce sujet, Stanga (1971) écrit:

"the relationship between most newsmen and prosecutors is so good that prosecutors are willing to release potentially prejudicial information to newsmen with the understanding, usually implicit, that newsmen ought not to publish such "off-the-record" information." (239)

De leur côté, les procureurs de la Couronne retirent certains bénéfices en s'adressant aux reporters, notamment en publicisant leurs procès afin de stigmatiser l'accusé, et en dissuadant la population en général à commettre des crimes semblables (Ericson et al., 1989). Cette symbiose existant entre les procureurs de la Couronne et les journalistes se reflète également dans les chroniques judiciaires. Une analyse de 
contenu des journaux publiés durant un mois en 1978 au Minnesota, réalisée par Drechsel et al. (1980), démontre effectivement que les articles traitant de procès en droit criminel incluent presqu'uniquement les propos de la Couronne. Suite à une analyse de contenu de journaux provenant de six villes aux États-Unis, Chermak (1998b) rapporte aussi que les articles de procès citent davantage les procureurs de la Couronne que les avocats de la défense. Cependant, la proportion de faits rapportés par la Couronne dans les chroniques judiciaires pourrait très bien être sous-estimée. Quelquefois, en effet, les reporters interrogent les procureurs de la Couronne au sujet d'un procès auquel ils n'ont pu assister (Ericson et al., 1989; Morissette, 1991). Suite à cette entrevue, ils rédigent leur chronique judiciaire en laissant l'impression qu'ils y ont bel et bien assisté, et que les propos retrouvés dans les articles proviennent d'eux-mêmes (Ericson et al., 1989). De cette façon, la version de la Couronne se présente subtilement dans les journaux.

En ce qui a trait aux avocats de la défense, la plupart d'entre eux évitent toute forme de publicité au sujet des procès dont ils ont la charge. Ils justifient ce choix en mentionnant l'impact négatif qu'une telle publicité pourrait avoir sur leurs clients. Selon eux, en effet, la publicité accordée à leurs clients constitue une peine additionnelle. D'autre part, les avocats de la défense conseillent à leurs clients de ne pas s'adresser à la presse. Cette directive ne semble pas contraindre le travail des journalistes puisque la plupart du temps, ceux-ci ne prennent même pas la peine d'interroger l'accusé (Ericson et al., 1989). À ce sujet, Fishman (1980) déclare: "In general, reporters in courtrooms will seek out lawyers, not their clients, as news sources" (62). Cette affirmation est bien illustrée dans l'ouvrage de Drechsel (1983), où 67\% des vingt-quatre reporters ont déclaré qu'au cours des six mois précédant l'entrevue, ils n'avaient eu aucun contact avec un accusé. Finalement, Chermak (1998b) remarque que les avocats de la défense se montrent plus réticents à discuter avec les journalistes, craignant dévoiler les stratégies qu'ils concoctent pour protéger leurs clients. Cette situation expliquerait notamment le peu d'information véhiculée en faveur des accusés dans les médias. 
En somme, les avocats de la Couronne constitueraient une source privilégiée pour les reporters, alors que les avocats de la défense livreraient très peu de commentaires. Pourtant, d'autres études viennent nuancer ces propos. Morissette (1991), par exemple, mentionne que les avocats des deux parties acceptent volontiers de collaborer avec les journalistes. Stanga (1971) rapporte aussi que les avocats de la défense entretiennent de bonnes relations avec les reporters et ce, afin de faire l'objet de publicité. Ces deux observations viennent à l'encontre de ce que la majorité des études ont rapporté. En général, par contre, les avocats de la défense sont moins sollicités que les procureurs de la Couronne. Stanga (1971) en vient également à cette conclusion malgré ses résultats contradictoires.

\subsubsection{3 - Les commis des palais de justice}

Les bureaux de réception et de consultation des palais de justice constituent une source-clé pour les journalistes. Non seulement les reporters peuvent-ils s'enquérir auprès des réceptionnistes pour être à l'affût de procès intéressants, mais ils peuvent également consulter la grille-horaire quotidienne des salles d'audience afin de planifier leur propre journée de travail (Drechsel, 1978, 1983). Les réceptionnistes sont peu (ou pas du tout) utiles pour donner de l'information spécifique sur le déroulement et les stratégies d'un procès; toutefois, ils représentent une source primordiale car sans eux, les reporters ne sauraient où se diriger pour trouver des procès dignes d'être mentionnés dans les journaux (Ericson et al., 1989). Ce n'est qu'à la suite de ce premier contact que les reporters peuvent se rendre à la salle d'audience pour observer le déroulement du procès et interviewer les avocats concernés. Morissette (1991) souligne bien le rôle-clé des commis:

"Le fait d'entretenir, avec les commis du greffe de chaque cour et des salles de dossiers, avec les secrétaires judiciaires (agissant comme soutien à l'audience), des rapports harmonieux, cordiaux et continus fait souvent toute la différence entre un reporter efficace et rapide et un autre qui se débrouille moins bien." (102) 
Les reporters se doivent donc d'entretenir des relations conviviales avec les commis. Ainsi, ces derniers seraient plus enclins à avertir les reporters d'un procès jugé digne d'intérêt (Drechsel, 1978).

\subsubsection{4 - Les journalistes}

Comme dans tout milieu couvert par les médias, les chroniqueurs judiciaires craignent d'omettre un événement intéressant qui fut rapporté dans un journal autre que celui auquel ils travaillent (dans le jargon du milieu journalistique, l'expression "se faire scooper" est utilisée pour illustrer cet incident) (Ericson et al., 1989; Grabosky et Wilson, 1989). Or, étant donné les contraintes organisationnelles imposées par les maisons de presse (explicitées plus loin), les reporters se consultent entre eux pour s'assurer qu'ils n'ont pas omis ou négligé la couverture d'événements importants. En effet, ils s'échangent de l'information afin de pouvoir rédiger leurs articles de journaux. Par ailleurs, ils se regroupent souvent dans la même salle d'audience afin d'assister à un procès suscitant l'attention de tous. De là provient l'expression "meute de journalistes", ou "pack journalism" en anglais (Ericson et al., 1989). Cette façon de procéder expliquerait notamment l'"uniformité aussi stérile qu'artificielle entre les médias concurrents" (Morissette, 1991: p.105). Toutefois, ce comportement grégaire et solidaire ne se retrouve pas dans tous les ouvrages. D'après ses observations auprès de reporters de la Californie, Fishman (1980) constate que certaines règles implicites limitent l'échange d'informations. Un reporter, par exemple, ne pouvait recevoir de l'information spécifique au sujet d'un événement qu'il n'avait pas couvert lui-même. De son côté, Morissette (1991) déclare que le phénomène des meutes de journalistes n'existe plus: "Bref, le régime de collaboration systématique a cessé, ... ce qui n'empêche pas les journalistes de s'aider mutuellement à l'occasion, notamment quand la journée est particulièrement chargée" (105-6). 


\subsubsection{5 - Les documents}

La dernière source d'importance pour les reporters constitue l'ensemble des documents fournis par les tribunaux, notamment les mandats, les rapports d'enquête et les retranscriptions des procès. La majorité des auteurs soulignent l'importance que revêt ce type de source. Drechsel (1978) rapporte que l'ensemble des reporters ayant répondu à son questionnaire utilisent régulièrement les documents des tribunaux. De plus, une autre étude de Drechsel (1983) démontre que plusieurs journalistes font usage des documents pour les fins de leur travail. En effet, tous les reporters échantillonnés avaient eu recours aux documents de façon quotidienne ou hebdomadaire au cours des six mois précédant l'entrevue. Schlesinger et Tumber (1994) confirment également que les reporters font un usage courant de la documentation retrouvée dans les tribunaux, de même que Chermak (1998b). Il n'y a qu'Ericson et al. (1989) qui rapportent des observations contraires à celles énoncées plus haut:

"In our observations, only basic public documents, such as fact sheets, the list of cases scheduled in each courtroom, and judgments from superior courts, were consulted. It was rare for court reporters to ask for other documents pertaining to a case, such as warrants and investigative reports." (71)

Cette contradiction pourrait s'expliquer par le sens que les chercheurs donnent au terme "documents" à travers les différentes études. Réfère-t-on strictement aux mandats, aux rapports d'enquête et aux retranscriptions de procès, ou inclue-t-on également les

grilles-horaire quotidiennes des salles d'audience? Si ces dernières font partie de ce que l'on entend par "documents" (comme semble l'indiquer l'étude de Drechsel (1983)), il n'est pas surprenant que les reporters répondent de la sorte et ce, étant donné qu'elles soient indispensables à la planification de leurs journées de travail (Morissette, 1991).

La consultation des différentes sources s'avère donc un pré-requis pour que les journalistes puissent rédiger leurs chroniques judiciaires. Comme il a été mentionné 
précédemment, des contraintes organisationnelles, exercées par les maisons de presse et les tribunaux, font en sorte que les reporters favorisent la collecte d'informations par l'entremise d'entretiens auprès de différentes sources et ce, au détriment de l'observation des procès. Les contraintes organisationnelles structurent non seulement la façon dont les reporters doivent recueillir l'information, mais elles déterminent aussi le type d'information qui doit être recueilli, ainsi que la manière de traiter et de présenter cette information dans les journaux.

1.1.4 - Les contraintes organisationnelles des maisons de presse et des tribunaux

Les maisons de presse et les tribunaux imposent des contraintes aux chroniqueurs judiciaires. Cinq types de contrainte seront abordés afin d'illustrer leur impact au niveau du travail des reporters et conséquemment, au niveau de la nature des chroniques judiciaires publiées dans les journaux.

\subsubsection{1 - La contrainte temporelle}

Dans un milieu où les éditeurs demandent aux reporters d'écrire deux ou trois articles intéressants chaque jour, il va de soi que ceux-ci ne peuvent perdre leur temps à observer le déroulement complet de séances ayant lieu dans les salles d'audience (Ericson et al., 1989). En conséquence, les journalistes se dirigent très rarement vers les salles d'audience et s'ils le font, ils n'y restent qu'une minute ou deux (Lotz, 1991). À ce sujet, Drechsel (1983) indique que sur l'ensemble des informations colligées par les chroniqueurs judiciaires, 29\% d'entre elles proviennent d'observations effectuées dans les salles d'audience. La plupart du temps, les journalistes se limitent à interviewer quelques informateurs-clés afin de rédiger leurs chroniques.

Les journalistes consacrent donc très peu de temps dans les salles d'audience, d'autant plus que certains d'entre eux occupent des fonctions autres que celle de 
chroniqueur judiciaire. Drechsel (1983) affirme que la grande majorité des reporters interviewés vaquaient à des tâches autres que celle qui leur était conférée dans les tribunaux. Son étude antérieure (Drechsel, 1978) démontre effectivement que 74\% des journalistes interviewés consacraient moins de 25\% de leur temps d'emploi à couvrir les tribunaux. Il en conclut donc ceci: "Clearly, court coverage for virtually all the reporters is a small part of their duties - probably part of a larger beat" (198). Le peu de temps passé dans les tribunaux contribuerait donc à un reportage superficiel des procès méritant une attention médiatique (Ericson et al., 1989; Lotz, 1991).

\subsubsection{2 - La contrainte spatiale}

Bien que les journalistes puissent observer le déroulement des procès dans les salles d'audience, de même que consulter différentes sources dans les palais de justice, une importante contrainte spatiale s'impose: l'inaccessibilité aux négociations de plaidoyer. En effet, les reporters ne peuvent accéder aux bureaux des juges et des avocats lors de ces négociations. Ils ne couvrent donc que les procès présentés au grand public (Ericson et al., 1989). En conséquence: "l'image que les médias projettent quotidiennement de l'appareil judiciaire est fondamentalement tronquée. Ils se rabattent, en fait, sur les rares procès qui ont lieu en bonne et due forme..." (Morissette, 1991: 224).

Même si le taux de négociations de plaidoyer s'avère fort élevé (Canada, 1987a; Poirier, 1987), le déroulement de cette pratique n'est pas dévoilé à la presse et au public pour diverses raisons: "Public scrutiny of plea-negotiation sessions is especially undesirable because these sessions are exploratory, unpredictable, and equivocal, compared to what transpires in trials within the highly structured context of the courtroom" (Ericson et al., 1989: 58). 
En interdisant l'accès aux journalistes lors de ces négociations, la légitimité de l'appareil judiciaire reste assurée. Et cette contrainte spatiale transparaît-elle dans les chroniques judiciaires? Seul Lotz (1991) a brièvement abordé le sujet en faisant une analyse de contenu de vingt quotidiens américains pour le mois de novembre 1989 (en excluant les parutions du dimanche). Voici ce qu'il en dit: "Court reports in the newspaper do mention plea bargaining in cases. I found well over a dozen stories that used the term "bargain"'" (132). Cette information s'avère peu utile en soi puisque l'auteur ne mentionne pas le nombre total de chroniques judiciaires inclus dans son analyse. Malgré tout, cette contrainte spatiale limite grandement l'accès aux sphères d'activité des tribunaux de juridiction criminelle. De cette façon, beaucoup d'information relative au fonctionnement des tribunaux demeure secrète et confidentielle (Ericson et al., 1989).

\subsubsection{3 - La contrainte législative}

Afin de s'assurer que les personnes accusées aient droit à un procès équitable, c'est-à-dire "un procès dont l'issue dépend uniquement de l'appréciation des preuves légalement produites par la poursuite et la défense" (Canada, 1987b: 20), les tribunaux disposent de plusieurs moyens qui restreignent la liberté d'expression des quotidiens. De cette façon, les risques de diffuser de l'information préjudiciable au bon déroulement du procès sont minimisés. Si, par exemple, les journalistes déclarent qu'un accusé a fait des aveux au sujet d'un crime commis, ils doivent le faire une fois cet élément reçu en preuve au procès de l'accusé (art. 542(2) du C.cr.). Le cas contraire, ils risquent fortement d'être accusés d'outrage au tribunal. De plus, certaines restrictions de publication existent, que ce soit selon les divers stages de procès (mise en liberté provisoire (art.517 du C.cr.), enquête préliminaire (art.539 et art.537(1)h) du C.cr.)) ou selon le statut des personnes impliquées (les mineurs par exemple (art.38 de la Loi sur les jeunes contrevenants)). Il faut également mentionner que le tribunal peut ordonner 
qu'un procès se tienne à huis clos (art.486(1) du C.cr.), même si cette mesure n'est appliquée qu'exceptionnellement (Morissette, 1991).

L'ensemble de ces dispositions législatives structure inévitablement le travail des chroniqueurs judiciaires. À ce sujet, Ericson et al. (1989) émettent le commentaire suivant: "The net effect of the invisible hand of the law is to lead reporters into selfpolicing, which makes them more conservative than the law itself" (66). Les auteurs rapportent que les journalistes consultent souvent des avocats afin de vérifier s'ils enfreignent ou non la loi. Dès que des doutes surviennent, il n'est pas rare qu'une partie de la chronique, ou la chronique toute entière, soit laissée de côté. En somme: "The law allowing publicity bans, when combined with the contempt-of-court- restrictions... had an inhibiting effect on what was reported" (Ericson et al., 1989: 68).

\subsubsection{4 - Le critère de "newsworthiness"}

Les journalistes doivent couvrir des événements dignes d'être publiés dans les journaux, ce qui correspond au critère de "newsworthiness". Ainsi, les journalistes recherchent des événements dramatiques et inattendus (Ericson et al., 1987) qui susciteront l'intérêt de la population. Morissette (1991) résume le contenu des médias de la façon suivante:

"Accent sur le spectaculaire, recherche du sensationnel, c'est-à-dire de ce qui, moyennant des procédés de séduction, produit une vive impression sur le public, plutôt que de privilégier la description clinique, l'exposé purement statistique ou scientifique de la réalité." (62)

Or, comme dans tout autre milieu couvert par les médias, le critère de "newsworthiness" structure la façon dont les chroniqueurs judiciaires recueillent leurs informations: "Their conceptions of newsworthiness narrowed, shaped, and framed what case was worth attending in the first place, and what aspects of that case should be 
attended to. Reporters considered only a tiny fraction of the cases being heard in court ..." (Ericson et al., 1989: 73).

Quels seraient donc les événements qui, du point de vue des reporters judiciaires, mériteraient l'attention d'une couverture journalistique? Tout d'abord, il semble que les procès impliquant la perpétration de meurtres ou de tentatives de meurtre aient préséance sur les procès où des crimes moins graves sont en litige. D'autre part, les procès de personnages publics ou de professionnels détenant du pouvoir et/ou du prestige (tels les juges, les avocats, les policiers et les entrepreneurs de compagnies importantes) suscitent l'attention des reporters (Lotz, 1991). Cette dernière constatation se retrouve également dans les études effectuées au Canada (Ericson et al., 1989; Rosenfeld, 1988; Tremblay, 1988).

Mis à part le statut de la personne et le type de crime impliqués, le type de sentence imposée constitue un facteur qui influence la couverture éventuelle d'un procès. Ainsi, Graber (1980) souligne que l'emprisonnement représente la peine la plus rapportée dans les chroniques judiciaires. Au Canada, Roberts (1988a) constate aussi que l'incarcération s'avère la peine de prédilection des quotidiens. Après avoir analysé un ensemble d'articles de journaux canadiens traitant de la détermination de la peine, il remarque effectivement que 70\% des articles mentionnent l'incarcération comme peine imposée. Les procès impliquant des crimes graves et conséquemment, de lourdes sentences, suscitent donc l'intérêt des reporters. Toujours au Canada, des entrevues réalisées auprès de journalistes démontrent qu'ils favorisent le reportage de procès où à leur avis, une peine extrêmement légère a été imposée (Canada, 1987a; Rosenfeld, 1988). Cette dernière observation découle de la tendance des journalistes à rapporter des événements inattendus (Ericson et al., 1987; Morissette, 1991). Mais qu'en est-il des facteurs associés à l'intensité de publication des procès paraissant dans les journaux? Bien que nous connaissions les types de procès qui ont le plus de chances d'être publiés, 
quels sont les éléments qui favorisent une publication plus intense d'un procès (par exemple, en terme de nombre d'articles consacrés par procès) que d'un autre?

Aucune recherche ne s'est affairée à répondre à cette question. Par contre, quatre études ont examiné les facteurs qui prédisent l'intensité de la couverture journalistique de crimes (Chermak 1998a) ou d'homicides perpétrés (Johnstone et al., 1994; Pritchard et Hughes, 1997; Wilbanks, 1984). Suite à une analyse de contenu de 1557 événements criminels publiés dans six quotidiens américains, Chermak (1998a) a cerné les caractéristiques des crimes, des accusés et des victimes qui pourraient prédire l'intensité de publication (espace alloué et score d'attention développé par l'auteur) de ces nouvelles. En effectuant des analyses de régression multiple, il a découvert que le nombre de victimes impliquées dans les événements constituait le meilleur prédicteur pouvant augmenter l'intensité de la publication. Aussi, le fait que le crime commis soit un meurtre influençait l'intensité de la publication dans le même sens. De leur côté, Johnstone et al. (1994) sont arrivés aux mêmes conclusions: en analysant les articles de deux journaux de Chicago traitant d'homicides perpétrés, ils se sont rendus compte que le nombre de victimes impliquées représentait le meilleur prédicteur qui augmentait l'intensité de publication (score d'attention développé par les auteurs). Pritchard et Hughes (1997) n'ont pu introduire cette variable dans leur recherche car il n'y avait pas assez d'événements d'homicide où plus d'une victime fut tuée ${ }^{1}$. Cependant, ils ont trouvé que les homicides impliquant une victime de sexe féminin faisaient l'objet d'un plus grand nombre d'articles. En ayant examiné des articles de journaux de Miami, finalement, Wilbanks (1984) a aussi trouvé que le meilleur prédicteur du nombre d'articles consacrés à une histoire d'homicide était le nombre de victimes impliquées. Bien que ces études ne se soient pas penchées sur l'intensité de la couverture journalistique des procès, il reste néanmoins que leurs résultats peuvent servir de point d'appui pour la présente recherche.

${ }^{1}$ Sur les 100 événements d'homicide qu'ils ont analysé à partir de deux journaux de Milwaukee, dans l'État du Wisconsin, seulement 4 d'entre eux comprenaient deux victimes, pour un total de 104 victimes. 


\subsubsection{5 - La simplification des nouvelles}

En plus de revêtir un caractère sensationnel, les événements rapportés dans les chroniques judiciaires doivent être simplifiés afin de susciter l'intérêt du public (Ericson et al., 1987). Les journalistes ont donc la tâche de transformer le discours légal, perçu comme étant hermétique et complexe, en langage accessible à la population en général. En conséquence:

"In fulfilling the requirements of news discourse, legal discourse is left out, relegated to its specialized professional contexts and texts. Inevitably, sources will complain they were misunderstood. They are literally correct when they say that in news coverage they are always taken out of context." (Ericson et al., 1989: 89-90)

Cette façon de simplifier pourrait notamment expliquer les erreurs retrouvées dans les chroniques judiciaires, erreurs que certains acteurs du champ judiciaire reprochent aux reporters (Ericson et al., 1989; Lotz, 1991). Morissette (1991) illustre éloquemment le processus de simplification des chroniques judiciaires: "Le plus souvent, un texte de trois feuillets, fouillé et détaillé, se trouve réduit, au moment de la publication, à un ou deux paragraphes" (55). Ainsi, la disparité existant entre le déroulement des événements et leur publication s'avérerait inéluctable puisque "[n]ews transforms the specialized knowledge of the legal institution into the common sense. ... Far from omission, selection, and distortion being problems for the news, they are fundamental to the news process..." (Ericson et al., 1989: 90).

1.1.5 - La corrélation entre la couverture journalistique des procès en droit criminel et la décision des jurés

Plusieurs études ont démontré que la publicité médiatique précédant un procès s'avère corrélée avec l'opinion des jurys au sujet de la culpabilité de l'accusé et de la sévérité de la sentence à imposer (Freedman et Burke, 1996; Greene et Loftus, 1984; 
Greene, 1990; Kerr, 1994; Kramer et al., 1990; Ogloff et Vidmar, 1994). Cependant, aucune étude ne s'est affairée à évaluer la corrélation entre la couverture médiatique du procès-même et les décisions des jurés. La raison en est fort simple: une fois assermentés, on demande aux jurés de ne pas lire ou écouter les propos rapportés par les médias au sujet du procès qui leur a été assigné2.

Lors de procès, la présence de journalistes peut toutefois modifier l'attitude du procureur de la Couronne envers l'accusé puisque "prosecutors' most common reaction to publicity, the existing research makes clear, is a desire to avoid being perceived as soft on criminals" (Pritchard, 1986: 145). Conséquemment, les procureurs de la Couronne risquent de se montrer intransigeants, sévères et rigoureux tout au long des audiences. Une telle attitude pourrait augmenter les probabilités à ce que les jurés donnent un verdict de culpabilité et/ou imposent une peine plus sévère qu'à l'habitude au condamné.

Nous allons maintenant aborder la révision judiciaire de la période d'inadmissibilité à la libération conditionnelle. Tout d'abord, le cadre légal de ce mécanisme de révision sera expliqué. Puis, quelques études à ce sujet seront traitées. Ces dernières permettront d'esquisser un portrait général de la présente recherche.

\section{2 - La révision judiciaire de la période d'inadmissibilité à la libération conditionnelle}

\subsection{1 - Le cadre légal}

Lors de l'abolition de la peine de mort au Canada, en 1976, de nouvelles peines furent instaurées pour les personnes condamnées pour meurtre. La peine de mort fut

\footnotetext{
2 À noter qu'au Canada, les journalistes ne peuvent interviewer les jurés puisque ces derniers n'ont pas le droit de discuter des délibérations ayant eu lieu durant le procès.
} 
alors remplacée par l'emprisonnement à perpétuité. Ainsi, les personnes condamnées pour meurtre au premier degré devaient purger vingt-cinq années d'emprisonnement avant d'être admissibles à la libération conditionnelle. En ce qui a trait aux individus condamnés pour meurtre au second degré, ils étaient contraints à purger une peine pouvant aller de dix à vingt-cinq années d'emprisonnement avant de pouvoir être éligibles à la libération conditionnelle. Ces modifications au Code criminel canadien furent accompagnées de l'article 745, maintenant devenu l'article 745.6: la révision judiciaire de la période d'inadmissibilité à la libération conditionnelle. Les dispositions de cet article permettent à une personne condamnée pour meurtre, et devant purger plus de quinze années d'emprisonnement, de demander une révision du délai d'inadmissibilité à la libération conditionnelle. La personne peut demander cette révision une fois qu'elle aura purgé au moins quinze années d'emprisonnement (art.745.6(1) du C.cr.). La requête doit être entendue en Cour supérieure, dans la province où la personne a été déclarée coupable du meurtre en question. Un jury détermine alors s'il y a lieu de réduire le délai de l'inadmissibilité à la libération conditionnelle du requérant en fonction des critères suivants: le caractère du requérant, sa conduite durant l'exécution de sa peine, la nature de l'infraction pour laquelle il a été condamné, tout autre renseignement fourni par un proche de la victime au moment de l'infliction de la peine ou lors de la présente audience, et tout autre renseignement que le juge estime utile dans les circonstances (art.745.63(1) du C.cr.). S'il y a effectivement lieu de réduire ce délai, le jury peut décider de diminuer le nombre d'années précédant le délai préalable à la libération conditionnelle, ou le supprimer (art.745.63(5) du C.cr.). Selon le moment choisi par le jury, le condamné pourra alors présenter une demande de libération conditionnelle à la Commission nationale des libérations conditionnelles.

Bien que cette disposition législative existe depuis 1976, des amendements y furent ajoutés récemment. Le projet de loi C-45, promulgué le 9 janvier 1997, a apporté trois modifications à la révision judiciaire. Depuis ce temps, les individus déclarés coupables de plus d'un meurtre ne peuvent présenter une demande de révision judiciaire 
(art.745.6(2) du C.cr.). De plus, un juge de la Cour supérieure doit démontrer qu'il existe une possibilité réelle à ce que la demande du requérant soit acceptée avant de procéder à la constitution du jury (art.745.61(1) du C.cr.). Finalement, le jury doit être unanime s'il décide qu'il y a lieu de réduire le délai préalable à la libération conditionnelle du requérant (art.745.63(3) du C.cr.). Auparavant, cette décision nécessitait au moins les deux tiers des membres du jury.

\subsection{2 - Les études à ce sujet}

La première demande de révision judiciaire a eu lieu au Québec, en 1987. Depuis ce temps, de plus en plus d'individus condamnés pour meurtre ont eu recours à cette requête et la tendance va en augmentant (O'Reilly-Fleming, 1991; Roberts, 1994a; Pilon, 1993/1997). Ces requêtes font parfois l'objet d'une couverture médiatique qui, d'après certains auteurs, n'explicite pas convenablement le processus de révision judiciaire (O'Reilly-Fleming, 1991; Roberts, 1994a) ou pire, lui attribue une publicité négative (Roberts, 1994a, 1994b, 1995, 1997). Par exemple, certains journalistes donnent l'impression qu'une fois acceptées, les demandes de révision judiciaire résultent en une éligibilité immédiate à la libération conditionnelle (Roberts, 1994a). D'autre part, il arrive souvent que les médias fassent paraître les commentaires provenant des membres de famille des victimes (Pilon, 1993/1997). En somme, Roberts (1997) affirme ceci à propos de la couverture médiatique de la révision judiciaire: "L'article 745 est peut-être devenu le plus controversé du Code criminel. Il a donné lieu à d'innombrables éditoriaux et lettres aux rédacteurs, de même qu'à une couverture négative abondante dans les médias" (4).

Étant donné que 31\% de la population canadienne s'informerait de l'imposition des sentences par l'intermédiaire de la presse écrite (Roberts, 1988b), l'impact d'une couverture journalistique négative au sujet de la révision judiciaire pourrait s'avérer nonnégligeable. Confrontée à un compte rendu déformé par les quotidiens, la population 
canadienne pourrait adopter une attitude négative à l'égard de la révision judiciaire (Roberts, 1994a). Ainsi, les Canadiens pourraient percevoir le processus de révision judiciaire de la même façon qu'ils perçoivent la détermination de la peine en général: celle-ci s'avérerait trop clémente envers les individus condamnés (Doob, 1995; Doob et al., 1998).

Or, quelques études ont examiné le rôle que joue le type de présentation du processus sentenciel sur l'opinion publique (Doob et Roberts, 1983; Roberts et Doob, 1990). Les résultats démontrent que plus les gens ont accès à l'information concernant l'imposition d'une peine quelconque (résumé des rapports pré-sentenciels et des retranscriptions du procès, etc.), plus ils considèrent que la peine administrée est appropriée. Les articles de journaux de ce même procès, qui ne donnent qu'une description partielle des faits relatés en Cour, engendrent un effet différent: ici, les gens s'opposent au jugement rendu car il est considéré comme trop clément (Doob et Roberts, 1983; Roberts et Doob, 1990). Ce phénomène pourrait également s'appliquer aux requêtes de révision judiciaire, en ce sens qu'une couverture journalistique partielle et erronée de celles-ci pourrait influencer l'opinion publique au sujet de ce mécanisme de révision (Roberts, 1994a).

L'ensemble de ces constatations amène Roberts (1994a) à formuler la recommandation suivante:

"The news media are of course critical to the formation of public opinion. A systematic content analysis of newspaper reports of applicants would provide a great deal of insight into the way that Judicial Review material has been conveyed to the public. An informal survey of newspaper stories has shown that a large number of misconceptions have been transmitted. In this context it would be interesting to conduct research... This would involve comparing the reactions of different groups of subjects, some of whom had read a newspaper account of an application, others who had read a more comprehensive summary of the information emerging during the course of the actual hearing." (12-3) 
La présente recherche consiste précisément à analyser la couverture journalistique accordée aux requêtes de révision judiciaire. Il ne s'agit pas d'évaluer l'opinion des gens face à ces requêtes, comme l'ont fait Doob et Roberts (1983) et Roberts et Doob (1990) à propos du processus sentenciel. Bien que ce type de recherche pourrait être intéressant en soi, nous sommes d'avis que l'examen du contenu journalistique s'avère une priorité. Comme Ericson et al. (1991) l'indiquent: "An understanding of news content is necessary before we can pose meaningful questions regarding the effects of content on consumers" (49). Ainsi, l'étude aura pour but d'évaluer la nature du contenu véhiculé par les quotidiens canadiens au sujet de ce mécanisme de révision.

\section{3 - Le cadre théorique}

Tout d'abord, le paradigme adopté pour la présente recherche est de type constructiviste (Berger et Luckmann, 1986; Guba et Lincoln, 1994). Ce paradigme considère la réalité comme étant relative et dépendante des individus ou des groupes qui la construisent (Guba et Lincoln, 1994). Les auteurs qui se sont penchés sur les rapports existant entre la justice criminelle et les médias ont souvent adopté ce paradigme (voir notamment Bailey et Hale, 1998; Barak, 1994; Ericson, 1995; Ericson et al., 1987, 1989, 1991; McCormick, 1995; Surette, 1984, 1992, 1998). Ils avancent que les médias jouent un rôle prépondérant dans la construction sociale de la réalité des individus et ce, particulièrement dans le domaine de la justice criminelle. Pour soutenir cette thèse, ils rappellent que 95\% des citoyens s'informent de la criminalité et de la justice criminelle par l'intermédiaire des médias de masse (Graber, 1980). Or, la réalité sociale de la justice criminelle, telle que véhiculée par les médias, ne reflète pas l'ensemble des événements qui surviennent réellement dans ce domaine (Surette, 1998). La presse écrite (l'un des médias de masse) tend à rapporter les événements sensationnels et ce, autant du côté des crimes que de celui des procès. D'autre part, les facteurs organisationnels et économiques des médias influencent l'information que ces derniers 
transmettent au grand public (Surette, 1998). C'est ainsi qu'Ericson et al. (1987) en viennent à concevoir les nouvelles: "News comes closer to mirroring the social and cultural reality of its own organization than to mirroring the events it reports on" (350).

Pour comprendre les principes qui sous-tendent la construction sociale de la justice criminelle telle que véhiculée par les médias, deux approches théoriques ont été proposées en sociologie: l'approche de l'idéologie dominante (Barlow et al., 1995; Fishman, 1978, 1980; Gorelick, 1989; Hall et al., 1978; Humphries, 1981; Soothill et Walby, 1991; Websdale et Alvarez, 1998) et l'approche institutionnelle (Chermak, 1994; Cohen \& Young, 1981; Ericson, 1991; Ericson et al., 1987, 1989, 1991; Schlesinger, 1989a, 1989b; Schlesinger et Tumber, 1994; Schlesinger et al., 1991). L'approche de l'idéologie dominante postule que les médias de masse contribuent à établir l'idéologie prônée par l'élite politique et économique en matière de justice criminelle (Hall et al., 1978). Ainsi, l'idéologie dominante préconiserait une approche "law and order" (Hall et al., 1978; Soothill et Walby, 1991) et tendrait à réduire le phénomène de la criminalité au niveau individuel (Ericson, 1991; Websdale et Alvarez, 1998). Le moment venu de couvrir un fait ou un événement rattaché à la justice criminelle, les médias rapporteraient constamment les propos tenus par les sources dominantes (les corps policiers, par exemple), ce qui assurerait leur hégémonie sur les autres sources (Ericson, 1991; Hall, 1986; Hall et al., 1978).

Certains chercheurs sont en désaccord avec cette approche (voir notamment Ericson, 1991; Ericson et al., 1987; Schlesinger et al., 1991). En effet, deux critiques majeures, interreliées, ont été reprochées: le "média-centrisme" et la tendance à prendre pour acquis l'hégémonie des sources officielles et dominantes des médias sur les sources alternatives (Ericson, 1991; Schlesinger et al., 1991). Le média-centrisme réfère au fait que les études adoptant l'approche de l'idéologie dominante ne se concentrent qu'à l'intérieur des organisations de médias de masse. Effectivement, les sources et le grand public ne sont pas abordés par les auteurs. Or, pour évaluer la transmission de 
l'idéologie dominante à partir des sources jusqu'aux médias, et pour vérifier si cette idéologie dominante est effectivement assimilée au sein du grand public, les tenants de cette approche auraient dû aborder ces deux sphères. D'autre part, étant donné que les sources n'ont pas été étudiées sous cet aspect, il devient risqué de postuler aveuglément que ce sont les sources dites officielles qui sont inéluctablement choisies par les médias (Ericson, 1991; Schlesinger et al., 1991). L'analyse de contenu de journaux, utilisée par les adeptes de l'approche de l'idéologie dominante, peut bel et bien démontrer qu'une idéologie dominante persiste et que les sources citées dans les journaux sont toujours les mêmes; par contre, les recherches ethnographiques dans le domaine (Chermak, 1994, 1995, 1998b; Ericson et al., 1987, 1989, 1991; Schlesinger et Tumber, 1994) ont modifié les postulats de cette approche.

L'approche institutionnelle (Ericson, 1991), qui découle des recherches ethnographiques complétées dans les milieux de la justice criminelle et des médias, considère que les médias ne reflètent pas uniquement les propos de l'idéologie dominante: au contraire, les médias chercheraient à communiquer un contenu diversifié, qui tient compte de la société pluraliste dans laquelle nous vivons (Ericson, 1991). Par ailleurs, l'approche institutionnelle avance que les sources des médias ne seraient pas nécessairement pré-déterminées. Bien que les défenseurs de cette approche reconnaissent que certaines sources ont de meilleures chances d'être entendues et rapportées, ils jugent toutefois qu'une lutte continue s'effectue entre les différentes sources (et à l'intérieur des sources elles-mêmes) afin qu'elles puissent paraître dans les médias (Ericson, 1991; Schlesinger et al., 1991). Ici donc, ce sont les contraintes organisationnelles, économiques et bureaucratiques qui sont prises en considération pour étudier le contenu journalistique traitant de la justice criminelle.

Deux approches théoriques prédominent donc afin de conceptualiser la couverture médiatique de la justice criminelle: une vision néo-marxiste confrontée à une vision pluraliste de la société (Schlesinger et Tumber, 1994). De plus, l'approche de 
l'idéologie dominante découle d'analyses de contenu, alors que l'approche institutionnelle provient de recherches ethnographiques effectuées dans les milieux de la justice criminelle et des médias de masse. En un sens, ces deux approches s'avèrent complémentaires: l'approche institutionnelle examine le processus de construction de la nouvelle, alors que l'approche de l'idéologie dominante étudie le produit fini, c'est-à-dire ce qui est véhiculé dans les médias.

Bien que l'analyse de contenu soit plus adéquate pour répondre aux questions de recherche de la présente étude (voir section suivante), nous n'adoptons pas l'approche de l'idéologie dominante pour autant. Tout en étant sensible aux idées développées par les deux approches théoriques, nous ne nous sentons pas obligés d'adhérer définitivement à l'une ou l'autre des approches et ce, étant donné la nature de nos questions de recherche.

\section{4 - Les objectifs}

De plus en plus de requêtes de révision judiciaire ont lieu. Cette disposition du Code criminel a fait l'objet de nombreux débats qui parfois, ont mené à une ou plusieurs modifications à l'article 745.6 du Code criminel. Avec l'adoption du projet de loi C-45, par exemple, de nouvelles conditions furent imposées afin de restreindre l'accessibilité à la révision judiciaire, et de limiter les possibilités à ce qu'il y ait éventuellement une réduction au délai préalable à la libération conditionnelle du requérant. Les modifications apportées découlent donc d'un mouvement social et politique qui vise à limiter, voire à abolir le recours à ce mécanisme de révision. Étant donné que les médias de masse influenceraient grandement l'opinion publique (Kent, 1985; Roberts, 1994a) et l'opinion des politiciens (Kent, 1985), il se pourrait que la couverture médiatique négative accordée aux requêtes de révision judiciaire (Roberts, 1994a, 1994b, 1995, 1997) ait contribué à établir le courant social et politique qui s'oppose à cette disposition législative. Or, aucune étude n'a effectué une analyse systématique du contenu rapporté par les médias lorsque de telles requêtes ont eu lieu devant les tribunaux. 
Nous nous proposons donc d'analyser la couverture journalistique de quotidiens canadiens au sujet des requêtes de révision judiciaire. La présente recherche s'attardera aussi bien à examiner la place qu'occupe la couverture journalistique de telles requêtes, qu'à analyser son contenu. De cette façon, il sera possible d'évaluer l'importance accordée aux requêtes dans les journaux. D'autre part, le contenu de cette couverture journalistique sera analysé afin d'identifier les sources les plus utilisées par les reporters, d'évaluer l'orientation générale (négative ou positive) du traitement journalistique des requêtes, et de vérifier l'exactitude des propos publiés au sujet de cette disposition législative. Puis, d'autres informations seront colligées pour cibler les facteurs qui favorisent une couverture journalistique intense des requêtes de révision judiciaire. Finalement, il sera question d'analyser la relation pouvant exister entre la couverture journalistique des requêtes de révision judiciaire et la décision des jurys de réduire éventuellement le délai d'inadmissibilité à la libération conditionnelle des requérants.

Les objectifs spécifiques sont donc:

- Évaluer l'étendue de la couverture journalistique des requêtes de révision judiciaire.

- $\quad$ Analyser le contenu rapporté dans la couverture journalistique des requêtes de révision judiciaire.

- $\quad$ Identifier les facteurs associés à l'intensité de la couverture journalistique des requêtes de révision judiciaire.

- $\quad$ Analyser la relation entre la couverture journalistique des requêtes de révision judiciaire et la décision des jurys de réduire le délai d'inadmissibilité à la libération conditionnelle. 
CHAPITRE II

MÉTHODOLOGIE 
Afin de compléter les objectifs de la recherche, une approche quantitative a été adoptée pour analyser les variables du corpus. Le présent chapitre décrira l'échantillon, la démarche méthodologique utilisée ainsi que les variables qui furent constituées avant qu'elles ne soient analysées.

\section{1 - L'échantillon à analyser}

L'échantillon est composé de deux banques de données distinctes, l'une étant tributaire de l'autre. En effet, une liste des requêtes de révision judiciaire ayant eu lieu au Canada a servi de point d'appui pour constituer la deuxième banque de données, c'està-dire l'ensemble des articles de quotidiens canadiens ayant traité de ces requêtes.

La liste des requêtes de révision judiciaire, fournie par le Service Correctionnel du Canada, comprend l'ensemble des requêtes qui ont eu lieu au Canada de 1987 (c'està-dire l'année où la première requête s'est déroulée au Canada) au 31 mai 1998. Au total, 92 requêtes figuraient sur la liste, avec plusieurs informations-clés pour chacune d'elles: le nom du requérant, le type de meurtre (meurtre au premier degré, meurtre au second degré, meurtre capital et meurtre non-capital) auquel le requérant avait été condamné, la province dans laquelle la requête de révision judiciaire avait eu lieu, la date où le jury avait rendu sa décision quant à la considération de réduire le délai d'inadmissibilité à la libération conditionnelle, ainsi que la nature de cette décision.

Certaines de ces informations-clés ont servi à constituer la deuxième banque de données, soit l'ensemble des articles de quotidiens canadiens ayant traité des requêtes de révision judiciaire. En effet, trois informations-clés (la province où la requête de révision judiciaire avait eu lieu, la date où la décision du jury avait été rendue, ainsi que le nom du requérant) ont été utilisées pour échantillonner les articles de quotidiens canadiens. 
2.1.1 - Critères d'échantillonnage des articles de quotidiens canadiens

Afin que l'échantillon des articles de quotidiens canadiens soit constitué de façon efficace, tout en étant le plus complet possible, trois critères d'échantillonnage ont été retenus:

2.1.1.1 - Échantillonner le(s) quotidien(s) de la province dans laquelle une ou plusieurs requêtes de révision judiciaire ont eu lieu (sauf pour le quotidien Globe and Mail)

La couverture journalistique de la justice criminelle se concentre beaucoup plus sur les événements survenus au niveau régional qu'à ceux survenus niveau national (Graber, 1979). De plus, Dussuyer (1979) a constaté que les journaux traitent davantage des procès au niveau des nouvelles locales qu'au niveau des nouvelles nationales ou internationales. Pour ces raisons, les articles à échantillonner devaient figurer dans les quotidiens de la province où la requête de révision judiciaire avait eu lieu. Étant donné son caractère pan-canadien, le quotidien Globe and Mail a fait exception à la règle. C'est donc le seul quotidien qui a été échantillonné pour l'ensemble des requêtes.

\subsubsection{2 - Échantillonner le(s) quotidien(s) le(s) plus lu(s) dans la province}

Seuls le ou les quotidiens les plus lus dans les provinces ont été sélectionnés. Pour évaluer ce critère, le tirage hebdomadaire des quotidiens (à partir des données du Audit Bureau of Circulations ${ }^{3}$ ) de chaque province fut pris en considération et ce, pour l'année ou les années où une ou des requêtes de révision judiciaire avaient eu lieu. Si les requêtes avaient eu lieu au cours de différentes années, la moyenne du tirage hebdomadaire de chaque quotidien de la province était calculée selon ces années.

${ }^{3}$ Le Audit Bureau of Circulations est une association à but non-lucratif qui fut fondée par des annonceurs, des agences de publicité et des éditeurs pour établir des standards objectifs ayant trait à la circulation de quotidiens et de revues. 
Le quotidien le plus lu de la province figurait automatiquement dans l'échantillon. Par la suite, le tirage de ses compétiteurs était examiné: si un ou plusieurs de ceux-ci tiraient l'équivalent de 66\% ou plus du journal le plus lu de la province, ils étaient également inclus dans l'échantillon. De cette façon, il devenait possible d'esquisser un portrait de la couverture journalistique des requêtes de révision judiciaire à partir des quotidiens les plus populaires de la province. Une seule exception à cette règle fut l'insertion du journal The Gazette (Montréal) pour le Québec. Bien que ce quotidien anglophone ne tire que l'équivalent de 58\% du journal le plus lu du Québec, il fut néanmoins intégré dans l'échantillon afin d'examiner d'éventuelles ressemblances ou différences entre les couvertures journalistiques anglophone et francophone d'une même province.

\subsubsection{3 - Établir une limite temporelle}

Pour chaque requête, seuls les articles de quotidien se situant dans la période s'échelonnant entre les trois semaines précédant la décision du jury, et les trois semaines suivant cette décision, étaient échantillonnés. Établir une limite temporelle de la sorte permet notamment d'amasser les articles de requêtes ayant duré plus que les quatre à huit jours habituels (Brown, 1992). D'autre part, cette limite temporelle permet de recueillir les récits secondaires (éditoriaux, dossiers, chroniques, lettres d'opinion) qui se situent majoritairement à la suite d'un événement important, l'événement important étant ici la décision du jury de considérer ou non une réduction du délai d'inadmissibilité à la libération conditionnelle. La figure 1 démontre que la majorité, sinon la quasi-totalité des articles traitant des requêtes de révision judiciaire ont dû être échantillonnés étant donné le peu d'articles retrouvés aux extrémités de la limite temporelle: 


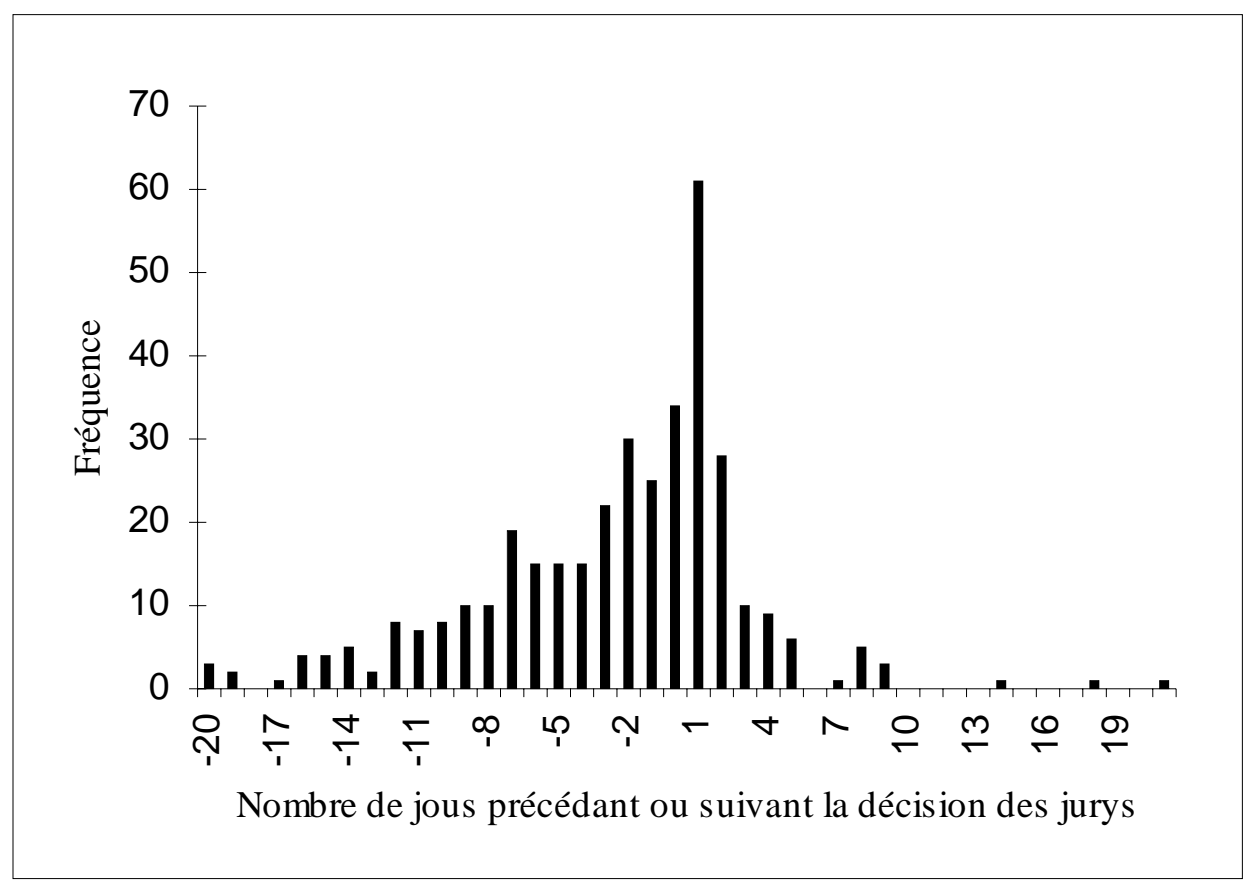

Figure 1:

Répartition des articles échantillonnés (n=365) selon les jours précédant ou suivant la décision du jury

En effet, la plupart des articles répertoriés ont été publiés une journée après que les jurys aient rendu leur décision (16.7\%), ou lors de cette journée-même (9.3\%). D'autre part, la majorité des articles (69.7\%) se situent entre les cinq journées précédant et suivant celle où les jurys ont rendu leur décision.

\subsection{2 - Méthode de cueillette}

La recherche des articles de quotidiens canadiens s'est effectuée de deux façons: par l'intermédiaire de CD-ROMs (Actualité-Québec, Canadian Newsdisc et Globe and Mail) ou par microfilms. La recherche par CD-ROM était favorisée en premier lieu. Plusieurs mots-clés étaient utilisés afin de repérer les articles traitant des requêtes de révision judiciaire dans les CD-ROMs: le nom du requérant, les mots "révision judiciaire", "révision et libération conditionnelle", "parole", "parole hearing", "judicial review" ainsi que la combinaison de ces mots-clés. Le fait de ne pas s'en tenir 
uniquement aux noms des requérants évitait que des erreurs de prononciation de noms, glissées dans la liste du SCC, nous empêchent de recueillir des articles au sujet des requêtes. Si les articles ne pouvaient être échantillonnés par CD-ROM (soit parce que le quotidien en question n'était pas répertorié dans les CD-ROMs, ou soit parce que les requêtes de révision judiciaire avaient eu lieu à une date antérieure aux années répertoriées dans les CD-ROMs), les microfilms des quotidiens étaient alors examinés. Le tableau I présente la répartition des articles traitant des requêtes de révision judiciaire selon les quotidiens des différentes provinces:

\section{Tableau I:}

Nombre d'articles échantillonés selon les quotidiens des différentes provinces

\begin{tabular}{cc}
\hline Province et quotidien & Nombre d'articles (\%) \\
\hline Colombie-Britannique & $\mathbf{5 9}(\mathbf{1 6 . 2 \% )}$ \\
The Vancouver Sun & $37(10.2 \%)$ \\
The Province, Vancouver & $22(6.0 \%)$ \\
Alberta & $\mathbf{5 7}(\mathbf{1 5 . 6 \% )}$ \\
Edmonton Journal & $14(3.8 \%)$ \\
Calgary Herald & $43(11.8 \%)$ \\
Saskatchewan & $\mathbf{7 9}(\mathbf{2 1 . 6 \% )}$ \\
The Leader Post, Regina & $28(7.7 \%)$ \\
The StarPhoenix, Saskatoon & $51(13.9 \%)$ \\
Manitoba & $\mathbf{9 ( 2 . 5 \% )}$ \\
Winnipeg Free Press & $9(2.5 \%)$ \\
Ontario & $\mathbf{3 8 ( 1 0 . 4 \% )}$ \\
The Toronto Star & $38(10.4 \%)$ \\
Québec & $\mathbf{6 7}(\mathbf{1 8 . 4 \% )}$ \\
Le Journal de Montréal & $28(7.7 \%)$ \\
La Presse, Montréal & $24(6.6 \%)$ \\
The Gazette, Montreal & $15(4.1 \%)$ \\
Nouveau-Brunswick & $\mathbf{7 ( 1 . 9 \% )}$ \\
The Times-Transcript, Moncton & $0(0.0 \%)$ \\
New Brunswick Telegraph Journal & $6(1.6 \%)$ \\
The Daily Gleaner, Fredericton & $1(0.3 \%)$ \\
Nouvelle-Écosse & $\mathbf{7 ( 1 . 9 \% )}$ \\
The Chronicle-Herald, Halifax & $7(1.9 \%)$ \\
Ensemble du Canada & $\mathbf{4 2 ( 1 1 . 5 \% )}$ \\
The Globe and Mail & $42(11.5 \%)$ \\
Total & $\mathbf{3 6 5 ( 1 0 0 \% )}$ \\
\hline
\end{tabular}


Comme le tableau I le démontre, les journaux de la Saskatchewan sont ceux qui ont publié le plus grand nombre d'articles au sujet des requêtes (21.6\% de l'échantillon), suivi de ceux du Québec (18.4\%) et de la Colombie-Britannique (16.2\%). À ce stade-ci, ces résultats s'avèrent peu révélateurs en soi étant donné que les informations au sujet du nombre de requêtes par province n'est pas indiqué. Il se pourrait donc que le nombre d'articles publiés selon les journaux des différentes provinces soit simplement tributaire du nombre de requêtes ayant eu lieu dans chacune de ces provinces. Cette hypothèse, qui dépasse l'analyse descriptive des données, sera vérifiée dans le prochain chapitre.

Le tableau I révèle toutefois que la couverture journalistique des requêtes ne se fait pas de façon uniforme à travers les quotidiens d'une même province. En Alberta, par

exemple, le journal Calgary Herald a publié trois fois plus d'articles (43 articles, soit 11.8\% de l'échantillon) que le Edmonton Journal (14 articles, 3.8\% de l'échantillon). Des résultats semblables, quoique moins prononcés, se retrouvent dans les autres provinces où plus d'un journal a été échantillonné. Ces différences pourraient notamment être attribuées à la distance séparant le siège social d'un quotidien et le Palais de justice où la requête de révision judiciaire a eu lieu. Les requêtes ayant eu lieu dans les Palais de justice situés à l'écart du siège social d'un journal auraient effectivement moins de chances d'être couvertes. Cependant, l'examen de la province du Québec laisse entendre que d'autres facteurs pourraient également expliquer ce manque d'uniformité. Les sièges sociaux du Journal de Montréal, de La Presse et The Gazette étant tous situés à Montréal, il aurait été prévisible de retrouver un nombre semblable d'articles pour chacun des quotidiens. Or, bien que le Journal de Montréal et La Presse aient publié une quantité semblable d'articles (28 et 24 articles, respectivement), le journal The Gazette n'en a publié que quinze. D'autres facteurs, comme la politique d'information particulière à un journal en ce qui a trait à la couverture des faits divers, ainsi que la tendance des médias anglophones du Québec à accorder une attention particulière aux 
procès qui mettent en cause un membre de leur communauté (Tremblay, 1988), pourraient expliquer la différence observée.

Le quotidien The Globe and Mail a publié 42 articles, et une bonne partie de ceux-ci traitent des requêtes ayant eu lieu en Ontario, où le siège social du quotidien est situé4. Tout au long de l'analyse des données, l'ensemble de ces articles ne sera pas traité séparément puisque la comparaison de la couverture journalistique à travers les différentes provinces a préséance sur la comparaison de cette couverture à travers les différents quotidiens. Conséquemment, les articles du Globe and Mail seront répartis selon la province où la requête publiée a eu lieu.

\section{2 - Les autres sources de données}

Mis à part les deux banques de données à analyser, soit la liste des requêtes de révision judiciaire et l'ensemble des articles de quotidiens canadiens, d'autres sources de données furent mises à contribution pour les fins de la présente recherche. Le texte de loi de l'article 745.6 du Code criminel, ainsi que des rapports de recherche, furent consultés afin de créer la variable "exactitude de l'article" (explicitée plus loin). D'autre part, une liste donnant des informations sur les palais de Justice où les requêtes du Québec ont eu lieu, fut utilisée pour compléter une analyse ayant trait à l'étendue de la couverture journalistique.

\section{3 - La démarche méthodologique}

Une fois les articles échantillonnés, les variables pouvaient maintenant être constituées selon deux unités d'analyse. La première unité d'analyse était la requête, et les variables se référant à celle-ci furent créées de deux façons. Tout d'abord, certaines variables furent créées à partir d'informations-clés provenant de la liste du SCC.

${ }^{4}$ Sur les 42 articles publiés, l'Ontario est la province qui fait l'objet du plus grand nombre d'articles (19), suivi de la Colombie-Britannique (15), du Québec (3), de la Nouvelle-Écosse (3) et de la Saskatchewan (2). 
D'autres variables, réservées uniquement aux requêtes ayant paru dans les journaux, furent constituées à partir d'une analyse de contenu des articles de quotidien. La deuxième unité d'analyse était l'article. Les variables concernant cette unité d'analyse furent créées à partir d'une analyse de contenu des articles.

Il existe plusieurs définitions de l'analyse de contenu (Kelly, 1986). Cependant, la plus répandue est celle de Berelson (1952/1971), qui la décrit comme une technique de recherche visant la description objective, systématique et quantitative du contenu manifeste des communications. L'analyse de contenu quantitative cherche à identifier les répétitions du corpus étudié (Ericson et al., 1991) en formant différentes catégories (ou variables) à analyser. C'est ce type d'analyse de contenu qui a été mis à contribution dans cette recherche.

\section{4 - Les variables}

Suite à la collecte de données concernant les requêtes de révision judiciaire ( $\mathrm{n}=92)$, les variables suivantes ont été retenues:

Tableau II:

Variables des requêtes de révision judiciaire

\begin{tabular}{c} 
Variables des requêtes \\
Province où la requête de révision judiciaire a eu lieu \\
Région du Canada où la requête de révision judiciaire a eu lieu \\
Palais de justice où la requête de révision judiciaire a eu lieu (pour le Québec seulement) \\
Année où la requête de révision judiciaire a eu lieu \\
Type de meurtre auquel le requérant a été condamné \\
cócision du jury de considérer ou non une réduction du délai d'inadmissibilité à la libération \\
conditionnelle \\
Rang chronologique de la requête dans la province où elle a eu lieu \\
\hline Variables réservées uniquement aux requêtes ayant été rapportées par les quotidiens \\
Province où la requête de révision judiciaire publiée a eu lieu \\
Région du Canada où la requête de révision judiciaire publiée a eu lieu \\
Année où la requête de révision judiciaire publiée a eu lieu \\
Nombre d'articles publiés par requête \\
Sexe de la victime impliquée dans le meurtre commis par le requérant \\
Type de victime impliqué dans le meurtre commis par le requérant \\
Type d'homicide commis pas le requérant
\end{tabular}


Nombre de victime(s) impliquée(s) dans le(s) meurtre(s) commis par le requérant Présence d'un groupe s'opposant à la requête de révision judiciaire du requérant

Orientation générale de la requête

Certaines des variables affichées au tableau II méritent d'être expliquées. La région du Canada où la requête de révision judiciaire a eu lieu représente une variable qui regroupe les provinces en cinq régions, soit la Colombie-Britannique, les Prairies (Alberta, Saskatchewan et Manitoba), l'Ontario, le Québec, et les provinces maritimes (le N.-B. et la N. -É.). Ce regroupement s'explique par le faible nombre de requêtes ayant eu lieu dans certaines provinces, ainsi que par la tendance des provinces regroupées ensemble à rapporter les requêtes de façon semblable (que ce soit en terme de pourcentage de requêtes publiées ou de nombre d'articles consacrés par requête). Le type de meurtre auquel le requérant a été condamné (meurtre au premier degré, meurtre au second degré, meurtre capital et meurtre non-capital) ${ }^{5}$ constitue un indice pour qualifier la gravité du geste commis par le requérant. Au sujet du rang chronologique de la requête, cette variable vise à quantifier le rang qu'occupe chaque requête dans sa province respective (première requête à avoir eu lieu dans la province, deuxième, etc.).

Au sujet des variables réservées aux requêtes ayant paru dans les quotidiens, cinq d'entre elles méritent d'être explicitées. Tout d'abord, le type de victime impliqué dans le meurtre se base sur le rôle social de celle-ci (Gaucher et Crow, 1994). Les deux catégories de la variable sont "policier" et "non-policier". Ensuite, le type d'homicide commis par le requérant réfère à une typologie d'homicide qui tient compte de la relation entre le meurtrier et la victime, ainsi que des fins poursuivies par l'auteur du crime (Cusson et Boisvert, 1994). Les catégories de cette variable sont donc: homicide familial ou passionnel, homicide querelleur et homicide associé à un autre crime commis par le meurtrier ${ }^{6}$. En ce qui a trait au nombre de victime(s) impliquée(s) dans le(s)

\footnotetext{
5 Jusqu’à 1976, les individus déclarés coupables de meurtre étaient déclarés coupables de meurtre capital ou de meurtre non-capital. Les individus déclarés coupables de meurtre capital étaient condamnés à la peine de mort, alors que ce n'était pas le cas pour les gens déclarés coupables de meurtre non-capital. ${ }^{6} \mathrm{~L}$ 'homicide familial ou passionnel se définit par la nature du lien qui unit les acteurs du crime, ce lien étant soit familial ou conjugal. L'homicide querelleur est précédé d'une bagarre ou d'une chicane, ou il résulte d'une vengeance. Il implique des gens qui ne sont pas unis par un lien conjugal ou familial.
} 
meurtre(s) du requérant, cette variable sépare le nombre de victimes en deux catégories: "une" ou "plusieurs"7. La présence d'un groupe s'opposant à la requête de révision judiciaire représente une variable dichotomique mesurée à partir des articles de quotidien. Pour chacune des requêtes, les articles étaient examinés pour déterminer s'ils faisaient mention ou non de la présence d'un groupe (groupe de pression, policiers, politiciens) s'opposant à la requête de révision judiciaire. Bien que les proches des victimes s'opposent habituellement à la requête de révision judiciaire, ils n'étaient pas considérés comme un groupe s'opposant à la requête, exception faite de ceux faisant partie d'un groupe de pression. Finalement, l'orientation générale de la requête indique si en général, les articles au sujet de la requête s'avéraient favorables, défavorables, équilibrés ou neutres par rapport au requérant et/ou à la réduction du délai d'inadmissibilité à la libération conditionnelle.

Voici maintenant la liste des variables évaluées pour les articles de quotidien :

Tableau III:

Variables des articles de quotidiens canadiens traitant des requêtes de révision judiciaire

Variables des articles

Nom du quotidien qui a publié l'article

Type de quotidien qui a publié l'article

Province où le quotidien est publié

Province où la requête traitée dans l'article a eu lieu Région du Canada où la requête traitée dans l'article a eu lieu

Nom du requérant auquel l'article réfère

Date où l'article a été publié

Type de récit de l'article

Redondance (oui/non) de l'article

Source dominante de l'article

Orientation de l'article

Orientation du titre de l'article

Exactitude de l'article

L'homicide associé à un autre crime se produit avant, pendant, ou immédiatement après la perpétration d'un crime comme le vol ou le viol (Cusson et Boisvert, 1994).

${ }^{7}$ Fait important à noter, certaines variables rattachées à la perpétration du meurtre (sexe de la victime, type de victime et type d'homicide), ainsi que la variable "type de meurtre auquel le requérant a été condamné", n'inclueront pas les requêtes publiées où plus d'un meurtre avait été commis par le requérant $(\mathrm{n}=11)$ et ce, afin d'éliminer le "bruit" qui pourrait être causé par celles-ci dans les catégories de ces variables. 
Au tableau III, le type de quotidien réfère à sa structure (tabloïd ou grand format). À ce sujet, il faut noter que les quotidiens de type tabloïd rapportent davantage d'événements ayant trait à la justice criminelle (Ericson et al., 1991), et le font de façon plus sensationnelle que le font les journaux de type grand format (Williams et Dickinson, 1993). Quant à la variable "type de récit de l'article", elle sépare les articles selon qu'ils soient des récits primaires (où l'on ne fait que rapporter un événement) ou des récits secondaires (où l'on s'affaire à répondre ou à réagir au sujet d'un récit primaire). Les récits secondaires incluent donc les éditoriaux, les chroniques, les dossiers ainsi que les lettres aux éditeurs (Voumvakis et Ericson, 1984; Martel, 1994). La variable "redondance de l'article" identifie les articles qui s'avèrent redondants d'un autre article. Ceux-ci n'ont donc pas été écrits intégralement par un chroniqueur judiciaire du journal, mais ont plutôt été copiés, partiellement ou complètement, à partir d'un article provenant d'un autre journal ou de la Presse Canadienne.

La source dominante de l'article réfère à la source à laquelle le journaliste fait le plus allusion, que ce soit en la citant ou en décrivant ce qu'elle a dit. La variable "orientation de l'article", quant à elle, détermine si le contenu manifeste de l'article est généralement favorable, défavorable, équilibré (favorable et défavorable) ou neutre (pas d'orientation) par rapport au requérant et/ou à la réduction du délai d'inadmissibilité à la libération conditionnelle (exemples de codification dans l'annexe A). Cette variable a fait l'objet d'un test de fiabilité intra-codeur, où le jugement du même codeur est mis à l'épreuve plus d'une fois à travers le temps (Kelly, 1986). Un mois après avoir été codifiés une première fois, en effet, les articles furent recodifiés pour évaluer la fiabilité des résultats. Selon les critères de Ciccheti et Sparrow (1981), il y a une excellente concordance entre les deux codifications $(\mathrm{K}=.80)^{8}$, d'autant plus que les articles codifiés différemment ont rarement passé d'un extrême à un autre (de favorable à

${ }^{8}$ Le kappa (K) de Cohen mesure le niveau de concordance existant entre deux codeurs lorsqu'ils codifient la même variable. La valeur du kappa peut varier de "0" à "1", "0" signifiant aucune concordance et "1" correspondant à une concordance parfaite entre les deux codeurs. Évidemment, la limite de la présente recherche provient du fait qu'un seul codeur a codifié les articles deux fois. 
défavorable, par exemple) ${ }^{9}$. Malgré tout, les articles ayant fait l'objet d'une codification différente au cours des deux évaluations $(n=52)$ furent codifiés par un deuxième juge. En tenant compte des trois codifications, une décision finale fut prise au sujet de leur orientation. Quant à l'orientation des titres des articles, cette variable n'a pas nécessité une deuxième codification étant donné qu'il était facile d'assigner la catégorie à laquelle chacun des titres appartenait (exemples de codification dans l'annexe A). Finalement, une dernière variable évalue l'exactitude des propos rapportés dans les articles au sujet de la nature et du fonctionnement de la révision judiciaire. Avec l'aide du texte de loi de l'article 745.6 du Code criminel et de rapports de recherche, les articles furent codifiés "exacts" ou "inexacts" au sujet de cette disposition légale.

Afin de préciser la répartition des articles telle que retrouvée dans le tableau I, le tableau IV décrit le type de récit et la redondance des articles selon les provinces où les requêtes ont eu lieu:

Tableau IV:

Type de récit et redondance des articles selon les provinces du Canada

\begin{tabular}{|c|c|c|c|c|c|c|c|c|c|}
\hline \multirow[t]{2}{*}{ Province } & \multicolumn{4}{|c|}{ Type de récit } & \multicolumn{4}{|c|}{ Redondance } & \multirow{2}{*}{$\begin{array}{c}\text { Total } \\
\text { (\% r.) }\end{array}$} \\
\hline & Primai & e (\% r.) & Seconda & ire (\% r.) & Non & (\% r.) & Oui & (\% r.) & \\
\hline C.-B. & 49 & (66\%) & 25 & (34\%) & 74 & $(100 \%)$ & 0 & $(0 \%)$ & $74(100 \%)$ \\
\hline Alberta & 57 & $(100 \%)$ & 0 & $(0 \%)$ & 53 & $(93 \%)$ & 4 & (7\%) & 57 (100\%) \\
\hline Saskatchewan & 75 & (93\%) & 6 & $(7 \%)$ & 62 & (77\%) & 19 & $(23 \%)$ & 81 (100\%) \\
\hline Manitoba & 9 & $(100 \%)$ & 0 & $(0 \%)$ & 9 & $(100 \%)$ & 0 & $(0 \%)$ & 9 (100\%) \\
\hline Ontario & 54 & (95\%) & 3 & (5\%) & 57 & (100\%) & 0 & (0\%) & 57 (100\%) \\
\hline Québec & 67 & (96\%) & 3 & (4\%) & 66 & $(94 \%)$ & 4 & (6\%) & 70 (100\%) \\
\hline N.-B. & 5 & (71\%) & 2 & (29\%) & 6 & $(86 \%)$ & 1 & $(14 \%)$ & 7 (100\%) \\
\hline N.-É. & 9 & $(90 \%)$ & 1 & $(10 \%)$ & 10 & $(100 \%)$ & 0 & $(0 \%)$ & $10(100 \%)$ \\
\hline Total & 325 & (89\%) & 40 & (11\%) & 337 & $(92 \%)$ & 28 & $(8 \%)$ & 365 (100\%) \\
\hline
\end{tabular}

${ }^{9}$ Sur les 365 articles, 52 d'entre eux (14.2\% de l'échantillon) ont fait l'objet d'une codification différente au cours des deux évaluations. Cependant, seulement un de ces articles (1.9\%) a fait l'objet d'une intracodification extrêmement divergente (ayant passé de "défavorable" à "favorable"). La majorité des articles divergents (60.0\%) ont été codifiés comme étant "défavorables" ou "favorables" au départ, pour ensuite être considérés comme étant "équilibrés" lors de la deuxième codification, ou l'inverse. 
La Colombie-Britannique se démarque des autres provinces par la proportion importante de récits secondaires (34\%), et ceux-ci se rattachent presqu'exclusivement à la requête de Clifford Olson ${ }^{10}$. Mis à par cette province, par contre, le nombre de récits secondaires de chaque province reste relativement bas. La Saskatchewan, de son côté, a publié beaucoup d'articles redondants (19 articles sur 81, soit 23\%) comparativement aux autres provinces. Selon que les requêtes aient eu lieu dans les environs de Regina ou de Saskatoon, en effet, il arrivait souvent que le journal de chacune de ces localités (The Leader Post et The StarPhoenix, respectivement) transmette les articles publiés au journal plus distant, qui les publiait à son tour. Cette façon de procéder explique partiellement le nombre élevé d'articles publiés dans les journaux de la Saskatchewan (voir tableau I).

\section{5 - Test statistique utilisé}

Afin de compléter certains objectifs de la recherche, le test du khi-carré fut utilisé pour vérifier l'existence éventuelle de relations significatives entre certaines variables. Ce test statistique indique s'il y a des différences significatives entre les catégories de la variable dépendante (la variable en colonne) lorsqu'elles sont croisées avec les catégories de la variable indépendante (la variable en rangée). Le test du khi-carré est un test d'indépendance et conséquemment, il ne fournit pas d'information au sujet du type d'association existant entre deux variables. En conséquence, les tests qui se révèleront significatifs seront interprétés afin d'identifier la nature des associations existant entre les variables.

\footnotetext{
${ }^{10}$ Clifford Olson est un meurtrier tristement célèbre qui a tué onze enfants ou adolescents en ColombieBritannique, lors des années 1980-1981.
} 


\section{6 - Limites de la recherche}

La présente recherche contient certaines limites. Tout d'abord, il semble que la liste des requêtes fournie par le SCC ne contienne pas la totalité des requêtes s'étant déroulées entre 1987 et le 31 mai 1998. Selon des informations fournies par une collègue, quelques requérants retrouvés dans la liste auraient eu droit à plus d'une requête alors que la liste ne fournissait que la date d'une requête pour chaque requérant. Le Code criminel stipule effectivement que dans les cas où le délai préalable à la libération conditionnelle du requérant n'est pas réduit, le jury peut soit fixer un délai d'au moins deux ans avant que le requérant puisse faire une nouvelle demande, soit décider que celui-ci ne pourra pas présenter une nouvelle demande (art.745.63(6) du C.cr.). Si le jury ne fixe pas un tel délai, ou s'il ne décide pas qu'une nouvelle demande ne pourra être présentée, il sera loisible au requérant de présenter une nouvelle demande au plus tôt deux ans après la date de la décision du jury concernant la première requête (art.745.63(8) du C.cr.). Ainsi, certains requérants se seraient déjà prévalus de cette possibilité sans que ce ne soit indiqué dans la liste du SCC. D'autre part, il semblerait que quelques requérants ne figurent tout simplement pas dans la liste. Donc, ce ne serait pas la totalité des requêtes qui auraient été étudiées dans la présente recherche, mais plutôt la totalité des requêtes qui figuraient dans la liste fournie par le SCC.

Une autre limite a pu s'insérer lors de la cueillette des données. En effet, il se pourrait que certains articles dans les CD-ROMs n'aient pas été échantillonnés faute de mots-clés adéquats. D'autre part, le fait qu'un seul juge ait codifié la majorité des articles au sujet de leur orientation (défavorable, favorable, équilibré, neutre) peut nuire à la fiabilité de cette variable. 


\section{CHAPITRE III}

ANALYSE DES RÉSULTATS 
Le chapitre d'analyse se divisera en quatre parties. Afin d'évaluer l'étendue de la couverture journalistique des requêtes de révision judiciaire, le pourcentage de requêtes publiées et l'intensité de publication de celles-ci seront examinées selon les différentes régions du Canada. D’autre part, l'évolution du nombre de requêtes et d'articles publiés au cours des années sera également décrite. Finalement, le pourcentage de requêtes publiées, ainsi que leur intensité de publication, seront analysés selon les types de quotidiens.

Deuxièmement, le contenu de la couverture journalistique des requêtes sera examiné sous trois facettes. En premier lieu, il s’agira d'évaluer la source dominante des articles selon les régions du Canada et selon les types de quotidiens. Puis, l'orientation des articles et de leur titre seront analysés en fonction des deux facteurs cihaut, ainsi qu'en fonction des caractéristiques liées aux homicides perpétrés par les requérants. La dernière facette, quant à elle, consistera à vérifier l'exactitude des articles selon l'ensemble de ces facteurs.

Troisièmement, les variables rattachées aux homicides perpétrés par les requérants, ainsi que certaines variables rattachées aux requêtes, seront croisées avec l'intensité de publication. Ainsi, il sera possible d'identifier les éléments qui pourraient favoriser une couverture journalistique plus intense des requêtes de révision judiciaire.

Finalement, des variables liées aux homicides et aux requêtes feront l'objet d'un croisement avec la décision des jurys de réduire ou non le délai d'inadmissibilité à la libération conditionnelle des requérants. De cette façon, les liens pouvant exister entre ces variables seront exposés et interprétés afin de mieux comprendre leur sens et leur nature. 


\section{1 - L'étendue de la couverture journalistique}

3.1.1 - L'étendue de la couverture journalistique selon les régions du Canada

L'étendue de la couverture journalistique des requêtes de révision judiciaire n'est pas uniforme à travers le Canada. Le tableau V fait état d'un premier aspect de cette étendue:

Tableau V:

Publication journalistique des requêtes de révision judiciaire selon les régions du Canada

\begin{tabular}{lccc}
\hline Région & $\begin{array}{c}\text { Nombre de requêtes } \\
\text { rapportées dans les } \\
\text { journaux }\end{array}$ & Nombre de requêtes & $\begin{array}{c}\text { Pourcentage de } \\
\text { requêtes rapportées } \\
\text { dans les journaux }\end{array}$ \\
\hline Colombie-Britannique & 5 & 7 & $71 \%$ \\
Les Prairies & 18 & 20 & $90 \%$ \\
Ontario & 12 & 20 & $60 \%$ \\
Québec & 10 & 43 & $23 \%$ \\
Provinces maritimes & 2 & 2 & $100 \%$ \\
Total & 47 & 92 & $51 \%$ \\
\hline
\end{tabular}

Tout d'abord, le tableau V démontre que dans les provinces maritimes et dans les Prairies, la totalité ou la quasi-totalité des requêtes ont été publiées (100\% et 90\%, respectivement). En ce qui a trait à la Colombie-Britannique et à l'Ontario, la majorité des requêtes ont paru dans les quotidiens échantillonnés (71\% et $60 \%$, respectivement). $\mathrm{Au}$ Québec, par contre, très peu de requêtes ont fait l'objet d'une couverture journalistique car seulement 10 des 43 requêtes (23\%) ont été publiées. À prime abord, il serait logique de croire que ce résultat soit tributaire d'un biais échantillonnal. Comme il a été indiqué dans le chapitre précédent, les trois quotidiens échantillonnés au Québec ont tous leur siège social à Montréal. Or, étant donné que la couverture journalistique de la justice criminelle se concentre majoritairement sur les événements survenus au niveau 
local (Graber, 1979), le faible pourcentage de requêtes publiées pourrait s'expliquer par le fait que plusieurs requêtes aient eu lieu ailleurs qu'à Montréal et ses environs, où les quotidiens n'auraient pas eu d'intérêt à les couvrir. Les résultats du tableau VI ne supportent toutefois pas cette hypothèse:

Tableau VI:

Requêtes de révision judiciaire au Québec selon les palais de Justice

\begin{tabular}{ccc}
\hline Palais de justice & Nombre de requêtes publiées & Nombre de requêtes \\
\hline Montréal & 4 & 17 \\
Longueuil & 0 & 3 \\
St-Jérôme & 3 & 4 \\
Joliette & 0 & 4 \\
Sept-Îles & 0 & 3 \\
Roberval & 1 & 1 \\
Trois-Rivières & 0 & 1 \\
Québec & 1 & 1 \\
Chicoutimi & 1 & 1 \\
Inconnu & 0 & 8 \\
Total & 10 & 43 \\
\hline
\end{tabular}

En effet, le tableau VI démontre qu'à Montréal et dans les environs (Longueuil, St-Jérôme et Joliette), seulement 7 requêtes sur une possibilité minimale de $28{ }^{11}$ (25\%) ont fait l'objet d'une couverture journalistique, contre 3 sur une possibilité minimale de 7 (43\%) pour le reste de la province. Il s'avère donc que la concentration des quotidiens dans la même ville, soit Montréal, ne puisse expliquer le faible pourcentage de requêtes publiées au Québec.

Une deuxième hypothèse pouvant expliquer ce faible pourcentage serait le nombre élevé de requêtes ayant eu lieu au Québec comparativement aux autres régions du Canada. Le tableau VI révèle qu’au Québec, effectivement, il y a eu 43 requêtes de révision judiciaire, l'Ontario et les Prairies suivant avec 20 requêtes chacune. Or, étant donné que "[l]a nouveauté, c'est la règle de base de toute activité journalistique, dans le

\footnotetext{
${ }^{11}$ Les requêtes où les palais de Justice s’avèrent “inconnus” ne se sont pas nécessairement déroulées à l'extérieur de Montréal et ses environs.
} 
domaine judiciaire comme dans les autres" (Tremblay, 1988: 14), il se pourrait que les premières requêtes de révision judiciaire au Québec aient fait l'objet d'une couverture journalistique, alors que les requêtes suivantes n'auraient pas été rapportées puisque "[l]es journalistes tentent d'éviter la lassitude de leurs lecteurs, auditeurs ou spectateurs en se gardant de rapporter de façon systématique des causes identiques qui deviennent vite sans intérêt" (idem: 14). En observant l'ordre chronologique des requêtes qui ont été publiées dans les régions de l'Ontario et du Québec (tableau VII), il semble qu'une telle hypothèse soit plausible:

Tableau VII:

Ordre chronologique des requêtes publiées selon les régions de l'Ontario et du Québec

\begin{tabular}{cccc}
\hline Région & \multicolumn{2}{c}{ Ordre chronologique de la requête publiée } \\
& 1ère à 10e & 11e et plus & Total \\
\hline Ontario & 8 & 4 & 12 \\
Québec & 6 & 4 & 10 \\
Total & 14 & 8 & 22 \\
\hline
\end{tabular}

Sur les dix premières requêtes qui ont eu lieu en Ontario, huit d'entre elles ont été rapportées alors que c'est le cas pour six d'entre elles au Québec. En ce qui a trait aux requêtes suivantes, seulement quatre requêtes sur une possibilité de dix pour l'Ontario, et de quatre sur une possibilité de trente-trois pour le Québec, ont fait l'objet d'une couverture journalistique. Ce dernier résultat renforce l'idée énoncée précédemment: lorsque le caractère de "nouveauté" des requêtes s'estompe, les journalistes sont moins portés à les couvrir.

Une deuxième façon d'analyser l'étendue de la couverture journalistique consiste à observer le nombre d'articles consacrés à chaque requête publiée. De cette façon, il devient possible d'évaluer l'intensité de publication rattachée aux requêtes qui ont été rapportées. Le tableau VIII résume ces résultats pour les différentes régions du Canada: 
Tableau VIII:

Nombre d'articles consacrés à chaque requête publiée selon les régions du Canada

\begin{tabular}{lcccccc}
\hline Région & 1 à 3 & 4 à 6 & 7 à 9 & 10 et plus & Total & Médiane \\
\hline Col.-Brit. & 3 & 1 & 0 & 1 & 5 & 3.0 \\
Prairies & 7 & 3 & 2 & 6 & 18 & 5.5 \\
Ontario & 6 & 3 & 1 & 2 & 12 & 3.5 \\
Québec & 6 & 2 & 1 & 1 & 10 & 1.5 \\
Maritimes & 0 & 0 & 1 & 1 & 2 & 8.5 \\
Total & 22 & 9 & 5 & 11 & 47 & 4.0 \\
\hline
\end{tabular}

Avant d'interpréter ce tableau, il faut préciser que les résultats exposés peuvent être tributaires du nombre de quotidiens échantillonnés dans chacune des provinces qui composent les régions. Comme il a été vu au tableau I du chapitre précédent, le nombre de quotidiens répertoriés pour chaque province n’est pas uniforme. Ainsi, le Québec et le Nouveau-Brunswick comprennent trois quotidiens chacun, alors que la ColombieBritannique, l'Alberta et la Saskatchewan en comprennent deux. Le Manitoba, l'Ontario et la Nouvelle-Écosse n'incluent qu'un quotidien échantillonné. Cette limite doit donc être prise en compte lors de l'analyse des résultats.

Les résultats du tableau VIII indiquent que près de la moitié des requêtes publiées (22 sur 47) ont fait l'objet d'une faible couverture journalistique (1 à 3 articles consacrés pour chaque requête), alors qu'un peu moins du quart d'entre elles (11 sur 47) ont fait l'objet d'une forte couverture (10 articles et plus). L'intensité de publication des requêtes varie d'une région à l'autre: dans les Prairies par exemple, huit requêtes sur dixhuit ont été rapportées sept fois et plus dans les quotidiens, contre seulement deux requêtes sur dix pour le Québec, une requête sur cinq pour la Colombie-Britannique et trois requêtes sur douze pour l'Ontario. À l'inverse, la majorité des requêtes publiées au Québec ont fait l'objet d'une faible couverture (6 requêtes sur 10), de même pour la Colombie-Britannique (3 sur 5). Le nombre médian d'articles consacrés par requête donne une vue d'ensemble de l'intensité de publication pour chaque région. Ainsi, les provinces maritimes consacrent un nombre médian de 8.5 articles par requête rapportée, suivi des Prairies (5.5), de l'Ontario (3.5) et de la Colombie-Britannique (3.0). Le 
Québec possède le nombre médian le plus bas, avec seulement 1.5 article destiné à chaque requête publiée. Donc, le Québec se démarque aussi bien par la faible proportion de requêtes publiées, que par le faible nombre d'articles consacrés à ces dernières.

\subsection{2 - L'étendue de la couverture journalistique selon les années}

L'étendue de la couverture journalistique des requêtes peut également être observée selon les années où elles ont été publiées. La figure 2 compare le nombre de requêtes ayant eu lieu au cours des années avec le nombre de requêtes ayant été publiées:

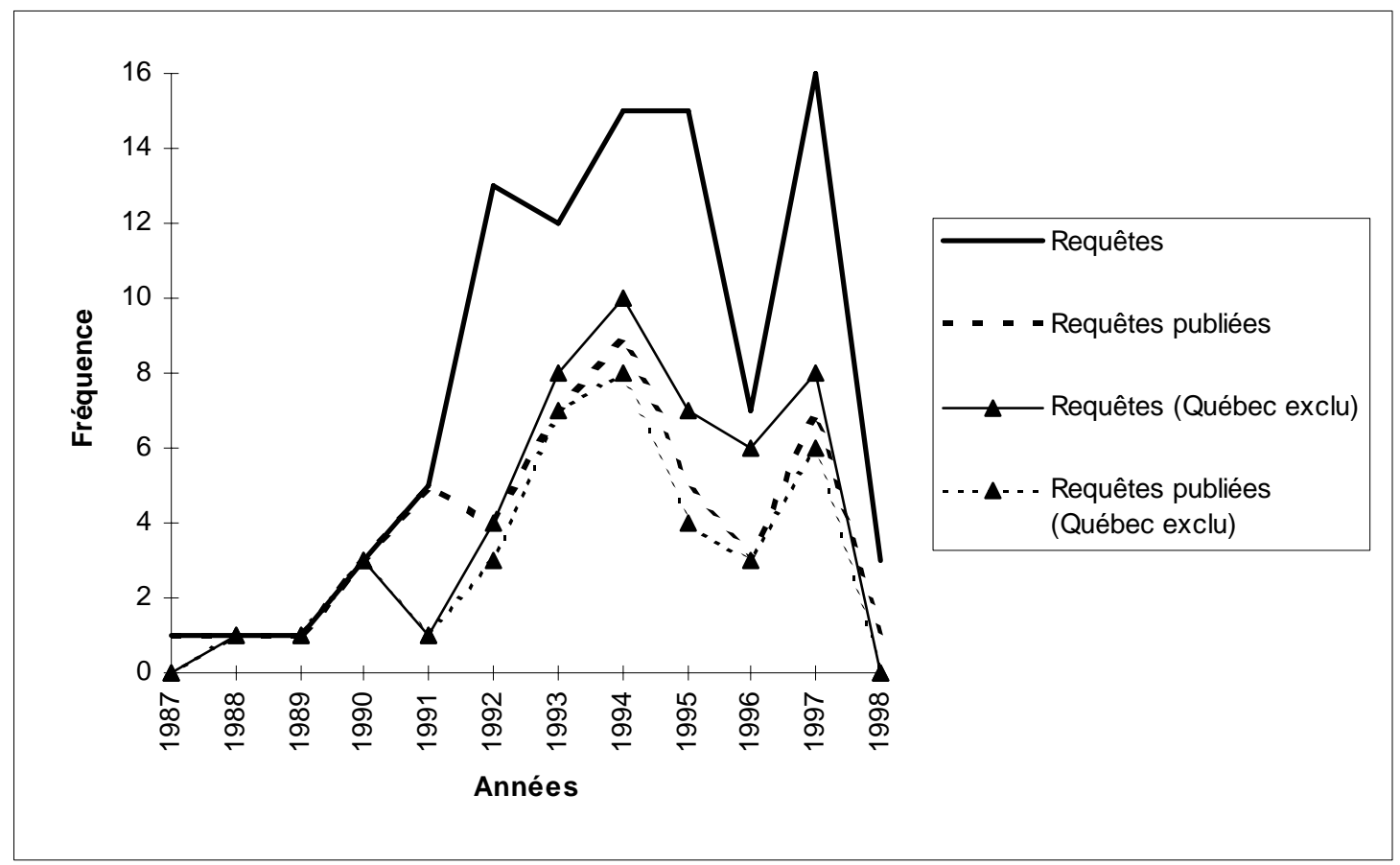

Figure 2:

Requêtes et requêtes publiées selon les années

La première requête a eu lieu en 1987, et il n'est pas surprenant de constater qu'elle fut rapportée dans les journaux. Il en va de même pour la totalité des requêtes 
s'étant déroulées entre 1988 et 1991. En 1992, par contre, seulement quatre requêtes sur treize ont fait l'objet d'une couverture journalistique. C'est donc à partir de cette année que l'"effet Québec" s'est fait ressentir car après 1991, il n'y a que deux années où plus de la moitié des requêtes sont apparues dans les quotidiens échantillonnés, soit 1993 (7 requêtes sur 12 , soit $58 \%$ ) et 1994 (9 sur 15 , soit $60 \%$ ).

Étant donné que le Québec occupe un statut particulier en terme de pourcentage de requêtes publiées, il s'avère nécessaire d'observer la tendance de publication annuelle en excluant cette région. En observant strictement les requêtes qui se sont déroulées ailleurs qu'au Québec, il appert que la quasi-totalité de celles-ci ont été publiées jusqu'en 1993. Puis à partir de 1994, une moins grande proportion de requêtes fut publiée chaque année. Cette dernière observation s'avère curieuse car le projet de loi C-45, qui modifiait la révision judiciaire de façon importante, a été présenté en 1996. Or, puisque les médias présentent les nouvelles en créant des thèmes (Fishman, 1980), il aurait été normal que la couverture journalistique du projet de loi, fort abondante tout au long de l'année 1996, soit accompagnée d'une couverture journalistique également abondante au sujet des requêtes. Au lieu, il semble que ce soit le critère de "nouveauté" (Tremblay, 1988) qui explique la proportion de requêtes publiées au cours de ces dernières années. Au fil du temps, en effet, les requêtes deviendraient de moins en moins dignes d'être mentionnées dans les journaux.

La figure 3 fait état de l'évolution temporelle de la couverture journalistique sous un autre angle. Cette figure montre que le nombre d'articles publiés par année suit une tendance semblable à celle du nombre de requêtes ayant eu lieu à chaque année. Mis à part les années 1988, 1989, 1995 et 1996, en effet, le nombre d'articles augmente d'une année à l'autre lorsque le nombre de requêtes augmente d'une année à l'autre aussi, et il diminue lorsque le nombre de requêtes va également en décroissant. D'autre part, la figure 3 démontre que la première requête à avoir eu lieu au Canada a fait l'objet d'une 
forte couverture journalistique ( $\mathrm{n}=45$ articles). Les années 1994 et 1997 se démarquent aussi des autres années par le nombre élevé d'articles qui ont été publiés. En 1994, une

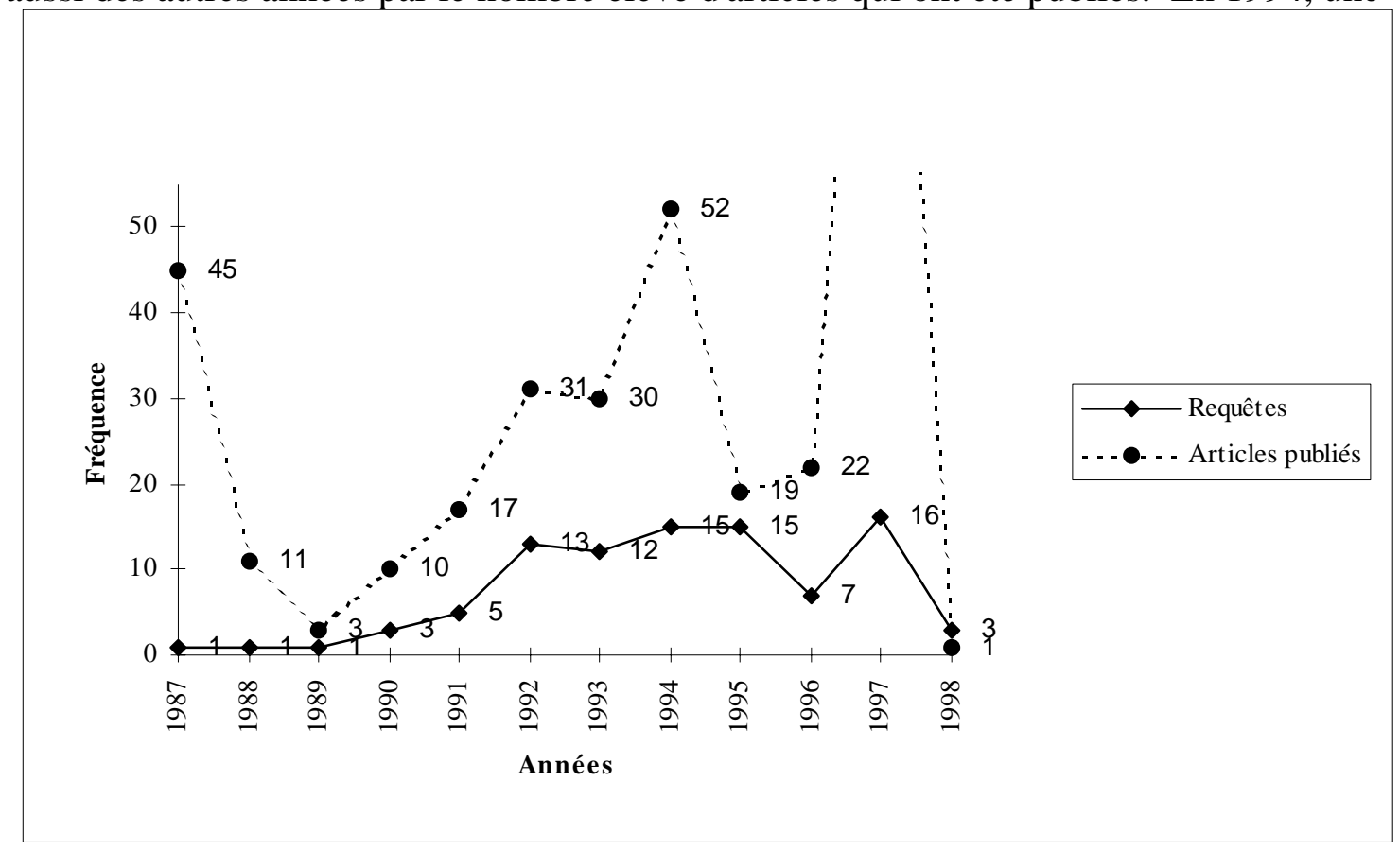

Figure 3:

Requêtes et articles publiés selon les années

requête se déroulant à Saskatchewan a été fortement publicisée $(n=30)$ et a fait l'objet de redondance, alors que le nombre élevé d'articles en 1997 (n=124, non-indiqué dans la figure 3) s'explique par la requête de Clifford Olson (n=63) ainsi que par deux autres requêtes fortement médiatisées en Alberta ( $\mathrm{n}=22)$ et en Saskatchewan $(\mathrm{n}=18)$.

\subsection{3 - L'étendue de la couverture journalistique selon les types de quotidien}

Selon la littérature, la couverture journalistique de la justice criminelle varie selon le type de quotidien: les journaux de type tabloïd rapportent davantage d'événements rattachés à la justice criminelle que les journaux de type grand format (Ericson et al., 1991). Dans le cadre de la présente recherche, il s'avère pertinent d'examiner les différences ou les ressemblances pouvant exister entre l'intensité de la 
couverture effectuée par les journaux tabloïds et celle effectuée par les journaux de type grand format. Le tableau IX permet d'observer ces résultats pour les deux régions où des quotidiens de type tabloïd furent échantillonnés, soit la Colombie-Britannique et le Québec:

Tableau IX:

Pourcentage de requêtes rapportées et nombre d'articles consacrés à chaque requête publiée selon les types de quotidiens de la Colombie-Britannique et du Québec

\begin{tabular}{cccccccc}
\hline Type de quotidien & $\begin{array}{c}\text { \% de requêtes } \\
\text { publiées }\end{array}$ & 1 à 3 & 4 à 6 & 7 à 9 & 10 et + & Total & Médiane \\
\hline $\begin{array}{c}\text { Tabloid } \\
\text { (The Province) }\end{array}$ & $29 \%$ & 1 & 0 & 0 & 1 & 2 & 11.0 \\
$\begin{array}{c}\text { Gr. form. } \\
\text { (Vancouver Sun) }\end{array}$ & $57 \%$ & 2 & 1 & 0 & 1 & 4 & 3.0 \\
\hline $\begin{array}{c}\text { Tabloid } \\
\text { (Journal de Montréal) } \\
\text { Gr. form. }\end{array}$ & $19 \%$ & 7 & 0 & 0 & 1 & 8 & 1.5 \\
$\begin{array}{c}\text { (La Presse) } \\
\text { Gr. form. }\end{array}$ & $5 \%$ & 0 & 1 & 0 & 1 & 2 & 7.5 \\
\hline (TheGazette) & $14 \%$ & 1 & 0 & 1 & 6 & 1.5 \\
\hline
\end{tabular}

Contrairement à ce que la littérature rapporte, le journal grand format de la Colombie-Britannique a rapporté davantage de requêtes (57\%) que le journal tabloïd (29\%). Au Québec, par contre, le Journal de Montréal a rapporté davantage de requêtes (19\%) que les deux journaux grand format, soit La Presse (14\%) et The Gazette (5\%). Comme il a été mentionné au chapitre précédent, le faible pourcentage de requêtes rapportées par The Gazette pourrait s'expliquer par la tendance des médias anglophones à couvrir davantage les procès qui mettent en cause un membre de leur communauté (Tremblay, 1988). Pour vérifier cette thèse, les noms des requérants du Québec furent observés et il s'est avéré que très peu d'entre eux possédaient un nom anglophone. Bien que cette information ne puisse confirmer qu'un requérant soit effectivement membre de la communauté anglophone, il reste néanmoins qu'elle constitue un indice allant vers cette direction. 
Le tableau IX indique aussi qu'indépendamment du type de quotidien, le nombre d'articles consacrés à chaque requête demeure sensiblement le même. Le tabloid Journal de Montréal et le quotidien grand format La Presse, par exemple, publient majoritairement peu d'articles pour chaque requête rapportée. Sur les huit requêtes rapportées par le Journal de Montréal, sept d'entre elles ont fait l'objet d'une faible couverture (de 1 à 3 articles) alors que c'est le cas pour quatre des six requêtes rapportées par La Presse. D'autre part, le nombre médian d'articles consacrés par requête est le même pour ces deux journaux (1.5). La médiane du quotidien grand format The Gazette est bien plus élevée (7.5), mais il faut tenir compte du fait qu'elle n'inclue le calcul que de deux requêtes. Finalement, il est intéressant de constater qu'en Colombie-Britannique comme au Québec, chacun des quotidiens a consacré dix articles et plus à une requête. La requête de Clifford Olson a fait l'objet de cette couverture en Colombie-Britannique alors qu'au Québec, ce fut celle de Réal Chartand, le premier meurtrier qui a eu droit à une requête au Canada en 1987. Ces résultats laissent donc l'impression suivante: lorsqu'une requête devient digne d'être mentionnée dans les journaux et ce, sans l'ombre d'un doute, chaque type de quotidien a tendance à publier beaucoup d'articles à son sujet.

\section{2 - Le contenu de la couverture journalistique}

\subsection{1 - La source dominante des articles}

Le tableau $\mathrm{X}$ fait état de la source dominante de chacun des articles échantillonnés. Les requérants représentent la source dominante qui paraît le plus fréquemment dans les articles (19\% de l'échantillon). À première vue, ce résultat va à l'encontre de ce qui fut rapporté lors de la recension des écrits. Effectivement, la littérature indique que les individus subissant leur procès ne sont pas souvent interrogés par les journalistes (Ericson et al., 1989; Fishman, 1980). Ici, par contre, la source dominante ne référait pas nécessairement à la source dominante interrogée par le 
reporter, mais plutôt à la source dont le journaliste faisait le plus allusion dans son article, que ce soit en la citant ou en décrivant ce qu'elle avait dit. Cette nuance doit donc être prise en compte car dans la présente recherche, les propos du requérant furent presqu'entièrement recueillis lorsqu'il témoignait durant la requête.

Tableau X:

Source dominante de l'article

\begin{tabular}{cc}
\hline Source dominante & Nombre d'articles (\%) \\
\hline Requérant & $69(19 \%)$ \\
Procureur de la Couronne & $39(11 \%)$ \\
Expert (psychiatre, psychologue ou criminologue) & $39(11 \%)$ \\
Proche d'une victime de meurtre & $37(10 \%)$ \\
Agent correctionnel, de gestion de cas & $34(9 \%)$ \\
ou de libération conditionnelle & \\
Avocat de la défense & $31(8 \%)$ \\
Document & $28(8 \%)$ \\
Proche du requérant & $19(5 \%)$ \\
Juge & $17(5 \%)$ \\
Policier & $9(3 \%)$ \\
Politicien & $8(2 \%)$ \\
Autre & $15(4 \%)$ \\
Inconnue & $20(5 \%)$ \\
Total & $365(100 \%)$ \\
\hline
\end{tabular}

Les procureurs de la Couronne et les experts constituent les sources dominantes qui reviennent le plus souvent après les requérants (11\% de l'échantillon chacun), suivis des proches d'une victime de meurtre (que ce soient les proches de la victime du meurtre commis par le requérant ou les proches d'une autre victime) (10\%), d'agents du système correctionnel (9\%) et d'avocats de la défense (8\%).

Dans les articles répertoriés, les experts témoignent principalement pour évaluer le risque que pourrait représenter le requérant une fois réintégré dans la société, alors que les agents du système correctionnel discutent de son comportement dans les pénitenciers qu'il a occupés. De leur côté, les procureurs de la Couronne, les proches d'une victime de meurtre ainsi que les policiers (24\% de l'échantillon en tout) représentent des sources dominantes évoquant une vision de la loi et l'ordre (Hall et al., 1978; Soothill et Walby, 1991), en ce sens qu'ils s'opposent majoritairement à la révision 
judiciaire du requérant. À l'inverse, les requérants, les avocats de la défense et les proches du requérant (32\%) sont presqu'unanimement favorables à une réduction éventuelle du délai d'inadmissibilité à la libération conditionnelle. Au niveau des sources dominantes, donc, les acteurs sociaux qui appuient ou s'opposent au processus de révision judiciaire sont présentés de façon à peu près égale.

La répartition des sources dominantes peut varier selon les régions du Canada et selon les types de quotidiens. Afin de croiser la source dominante des articles avec ces variables, seules les six sources les plus rapportées (requérant, procureur de la Couronne, expert, proche d'une victime de meurtre, agent correctionnel et avocat de la défense) furent conservées pour les prochaines analyses.

\subsubsection{1 - La source dominante des articles selon les régions du Canada}

Les sources dominantes ne sont pas réparties de façon uniforme à travers les différentes régions du Canada. Le tableau XI indique la manière dont les sources sont dispersées:

Tableau XI:

Source dominante de l'article selon les régions du Canada

\begin{tabular}{cccccccc}
\hline Région & $\begin{array}{c}\text { Requé- } \\
\text { rant } \\
\text { (\% rang.) }\end{array}$ & $\begin{array}{c}\text { Procureur } \\
\text { de la } \\
\text { Couronne } \\
\text { (\% rang.) }\end{array}$ & $\begin{array}{c}\text { Avocat de } \\
\text { la défense }\end{array}$ & $\begin{array}{c}\text { Agent } \\
\text { cor- } \\
\text { (\% rang.) }\end{array}$ & $\begin{array}{c}\text { Expert } \\
\text { (\% rang.) }\end{array}$ & $\begin{array}{c}\text { Proche de } \\
\text { la victime }\end{array}$ & Total \\
C.-B. & 9 & 5 & 4 & 0 & 4 & 10 & rang.) \\
(\% rang.) & (\% rang.) \\
Prairies & $(28 \%)$ & $(16 \%)$ & $(13 \%)$ & $(0 \%)$ & $(13 \%)$ & $(31 \%)$ & $(100 \%)$ \\
& 23 & 18 & 15 & 20 & 15 & 18 & 109 \\
Ontario & 16 & $(17 \%)$ & $(14 \%)$ & $(18 \%)$ & $(14 \%)$ & $(17 \%)$ & $(100 \%)$ \\
& $(37 \%)$ & $(14 \%)$ & $(14 \%)$ & $(9 \%)$ & $(9 \%)$ & $(16 \%)$ & $(100 \%)$ \\
Québec & 18 & 7 & 6 & 9 & 13 & 0 & 53 \\
& $(34 \%)$ & $(13 \%)$ & $(11 \%)$ & $(17 \%)$ & $(25 \%)$ & $(0 \%)$ & $(100 \%)$ \\
Marit. & 3 & 3 & 0 & 1 & 3 & 2 & 12 \\
& $(25 \%)$ & $(25 \%)$ & $(0 \%)$ & $(8 \%)$ & $(25 \%)$ & $(17 \%)$ & $(100 \%)$ \\
Total & 69 & 39 & 31 & 34 & 39 & 37 & 249 \\
& $(28 \%)$ & $(16 \%)$ & $(12 \%)$ & $(14 \%)$ & $(16 \%)$ & $(15 \%)$ & $(100 \%)$ \\
\hline
\end{tabular}


Tout d'abord, le requérant constitue une source dominante qui revient plus fréquemment en Ontario (37\%) et au Québec (34\%) que dans les Prairies (21\%) et en Colombie-Britannique (28\%). Le requérant paraît également moins souvent comme source dominante dans les provinces maritimes (25\%), mais le peu d'articles retrouvés dans cette région $(n=12)$ limite la validité de cette interprétation. D'autre part, il est surprenant de constater qu'aucun proche d'une victime ne figure comme source dominante au Québec, alors que c'est le cas pour une part non-négligeable des articles de la Colombie-Britannique (31\%) et dans une moins grande mesure, de ceux de la région des Prairies (17\%) et de l'Ontario (16\%). En Colombie-Britannique, ce pourcentage élevé découle de la requête de Clifford Olson, où plusieurs membres de famille des onze victimes se sont présentés pour y assister. Dans cette même province, également, aucun agent du système correctionnel n'apparait comme source dominante d'un article, contre 18\% pour les articles des Prairies et 17\% pour ceux du Québec. Par ailleurs, le quart des articles du Québec ont un expert comme source dominante, comparativement à 9\% en Ontario, 13\% en Colombie-Britannique et 14\% dans les Prairies.

\subsubsection{2 - La source dominante des articles selon les types de quotidien}

Il est également possible que la source dominante des articles varie selon le type

de quotidien. Étant donné que les tabloïds donnent un ton plus sensationnel aux événements de justice criminelle (Williams et Dickinson, 1993), les sources qu'ils utilisent pourraient effectivement être choisies afin d'impressionner les lecteurs. Le tableau XII donne une idée quant à la validité de cette hypothèse.

Les résultats vont dans le même sens que l'idée postulée. En ColombieBritannique, effectivement, sept articles sur seize du journal tabloïd ont pour source dominante les proches d'une victime de meurtre, contre trois articles sur onze pour le journal de type grand format. En rapportant davantage les propos des proches de la victime d'un meurtre, le tabloïd met l'accent sur la dimension émotionnelle (tristesse, 
colère) associée aux requêtes. Comme Ericson et al. (1991) le rapportent: "the popular newspaper... gives more emphasis to emotive... understanding" (39). Ainsi, les lecteurs ont l'occasion d'avoir une idée de ce que ces gens éprouvent depuis le décès de leur proche, et conséquemment, de ce qu'ils pensent de la révision judiciaire: "Playing on the heart, and on lower regions of the anatomy, popular newspapers are able to effect a sense of what it is 'really' like to be involved in a situation" (idem: 35).

\section{Tableau XII:}

Source dominante de l'article selon les types de quotidien de la Colombie-Britannique et du Québec

\begin{tabular}{cccccccc}
\hline $\begin{array}{c}\text { Type de } \\
\text { quotidien }\end{array}$ & $\begin{array}{c}\text { Requé- } \\
\text { Rant }\end{array}$ & $\begin{array}{c}\text { Procureur } \\
\text { de la } \\
\text { Couronne }\end{array}$ & $\begin{array}{c}\text { Avocat de } \\
\text { la défense }\end{array}$ & $\begin{array}{c}\text { Agent } \\
\text { cor- } \\
\text { rectionnel }\end{array}$ & Expert & $\begin{array}{c}\text { Proches } \\
\text { de la } \\
\text { victime }\end{array}$ & Total \\
\hline $\begin{array}{c}\text { Tabloid } \\
\text { (ThePro.) }\end{array}$ & 4 & 2 & 1 & 0 & 2 & 7 & 16 \\
$\begin{array}{c}\text { Gr. Form. } \\
(\text { Van.Sun })\end{array}$ & 2 & 2 & 2 & 0 & 2 & 3 & 11 \\
$\begin{array}{c}\text { Tabloid } \\
(J . \text { de } M .)\end{array}$ & 6 & 4 & 4 & 1 & 5 & 0 & 20 \\
$\begin{array}{c}\text { Gr. Form. } \\
(\text { La Pres.) }\end{array}$ & 6 & 2 & 2 & 5 & 4 & 0 & 19 \\
$\begin{array}{c}\text { Gr. Form. } \\
(\text { TheGaz.) } \\
\text { Total }\end{array}$ & 4 & 1 & 0 & 3 & 4 & 0 & 12 \\
\hline
\end{tabular}

Au Québec, seulement un article sur vingt du journal tabloïd a comme source dominante les agents du système correctionnel, contre cinq sur dix-neuf pour le quotidien grand format La Presse et trois sur douze pour The Gazette. Comme ces trois journaux ont couvert à peu près toutes les mêmes requêtes et ce, durant les mêmes journées d'audience, il se pourrait que le journal de type tabloïd ait moins tendance à rapporter les propos des agents du système correctionnel. Ces propos traitent habituellement des progrès du requérant durant son incarcération, ainsi que du climat difficile qui règne dans les pénitentiers. Au lieu, le Journal de Montréal met l'accent sur les propos des procureurs de la Couronne (quatre articles sur vingt) et des avocats de la défense (quatre articles sur vingt), deux sources continuellement retrouvées dans les tribunaux de juridiction criminelle. 
En ce qui concerne les deux provinces, cependant, les deux types de quotidiens accordent une importance semblable aux requérants. Donc, bien que les journaux tabloïds rapportent davantage les propos des victimes ou des acteurs sociaux habituellement retrouvés dans les tribunaux, l'importance qu'ils accordent aux requérants est la même que celle des journaux de type grand format. Cette remarque s'applique également aux experts en tant que source dominante.

\subsection{2 - L'orientation des titres et des articles}

La section qui suit constitue l'une des parties les plus importantes de la présente recherche. Comme il a été mentionné dans le premier chapitre, de nombreux ouvrages indiquent que les requêtes de révision judiciaire ont fait l'objet d'une couverture médiatique négative (Roberts, 1994a, 1994b, 1995, 1997). Cependant, aucun de ces ouvrages n'a évalué l'orientation (favorable, défavorable, équilibrée ou neutre) de la couverture de façon systématique. Nous nous proposons donc, à partir du corpus échantillonné, d'analyser l'orientation des titres et des articles au sujet du requérant et/ou de la réduction du délai d'inadmissibilité à la libération conditionnelle. La raison pour laquelle nous nous sommes également attardés aux titres des articles provient du fait que "many readers may not read beyond the headlines of many news stories, and their knowledge or opinions of certain news events may thus depend on... such headlines" (Marquez, 1980: 31). Ainsi, l'examen de ces deux unités d'analyse (le titre et l'article) permettra d'évaluer systématiquement le type de couverture journalistique consacré aux requêtes de révision judiciaire.

\subsubsection{1 - L'orientation des titres et des articles selon les régions du Canada}

Le tableau XIII fait état de l'orientation des titres ${ }^{12}$ et des articles selon les cinq régions du Canada:

\footnotetext{
12Les titres "équilibrés" ont été exclus des analyses étant donné leur faible nombre ( $\mathrm{n}=6$ ).
} 
Tableau XIII:

Orientation du titre et de l'article selon les régions du Canada

\begin{tabular}{|c|c|c|c|c|c|c|c|c|c|}
\hline \multirow[b]{2}{*}{ Région } & \multicolumn{4}{|c|}{ Orientation du titre } & \multicolumn{5}{|c|}{ Orientation de l'article } \\
\hline & $\begin{array}{l}\text { Défav. } \\
\text { (\% r.) }\end{array}$ & $\begin{array}{c}\text { Faveur } \\
\text { (\% r.) }\end{array}$ & $\begin{array}{c}\text { Neutre } \\
\text { (\% r.) }\end{array}$ & $\begin{array}{c}\text { Total } \\
\text { (\% r.) }\end{array}$ & $\begin{array}{l}\text { Défav. } \\
\text { (\% r.) }\end{array}$ & $\begin{array}{c}\text { Faveur } \\
\text { (\% r.) }\end{array}$ & $\begin{array}{l}\text { Équil. } \\
\text { (\% r.) }\end{array}$ & $\begin{array}{c}\text { Neutre } \\
\text { (\% r.) }\end{array}$ & $\begin{array}{c}\text { Total } \\
\text { (\% r.) }\end{array}$ \\
\hline C.-B. & $\begin{array}{c}31 \\
(42 \%)\end{array}$ & $\begin{array}{c}3 \\
(4 \%)\end{array}$ & $\begin{array}{c}40 \\
(54 \%)\end{array}$ & $\begin{array}{c}74 \\
(100 \%)\end{array}$ & $\begin{array}{c}43 \\
(58 \%)\end{array}$ & $\begin{array}{c}13 \\
(18 \%)\end{array}$ & $\begin{array}{c}7 \\
(10 \%)\end{array}$ & $\begin{array}{c}11 \\
(15 \%)\end{array}$ & $\begin{array}{c}74 \\
(100 \%)\end{array}$ \\
\hline Prairies & $\begin{array}{c}37 \\
(26 \%)\end{array}$ & $\begin{array}{c}27 \\
(19 \%)\end{array}$ & $\begin{array}{c}78 \\
(55 \%)\end{array}$ & $\begin{array}{c}142 \\
(100 \%)\end{array}$ & $\begin{array}{c}51 \\
(35 \%)\end{array}$ & $\begin{array}{c}40 \\
(27 \%)\end{array}$ & $\begin{array}{c}46 \\
(31 \%)\end{array}$ & $\begin{array}{c}10 \\
(7 \%)\end{array}$ & $\begin{array}{c}147 \\
(100 \%)\end{array}$ \\
\hline Ontario & $\begin{array}{c}10 \\
(18 \%)\end{array}$ & $\begin{array}{c}12 \\
(21 \%)\end{array}$ & $\begin{array}{c}34 \\
(61 \%)\end{array}$ & $\begin{array}{c}56 \\
(100 \%)\end{array}$ & $\begin{array}{c}9 \\
(16 \%)\end{array}$ & $\begin{array}{c}18 \\
(32 \%)\end{array}$ & $\begin{array}{c}21 \\
(37 \%)\end{array}$ & $\begin{array}{c}9 \\
(16 \%)\end{array}$ & $\begin{array}{c}57 \\
(100 \%)\end{array}$ \\
\hline Québec & $\begin{array}{c}6 \\
(9 \%)\end{array}$ & $\begin{array}{c}19 \\
(27 \%)\end{array}$ & $\begin{array}{c}45 \\
(64 \%)\end{array}$ & $\begin{array}{c}70 \\
(100 \%)\end{array}$ & $\begin{array}{c}7 \\
(10 \%)\end{array}$ & $\begin{array}{c}37 \\
(53 \%)\end{array}$ & $\begin{array}{c}15 \\
(21 \%)\end{array}$ & $\begin{array}{c}11 \\
(16 \%)\end{array}$ & $\begin{array}{c}70 \\
(100 \%)\end{array}$ \\
\hline $\begin{array}{l}\text { Mariti- } \\
\text { Mes }\end{array}$ & $\begin{array}{c}6 \\
(35 \%)\end{array}$ & $\begin{array}{c}4 \\
(24 \%)\end{array}$ & $\begin{array}{c}7 \\
(41 \%)\end{array}$ & $\begin{array}{c}17 \\
(100 \%)\end{array}$ & $\begin{array}{c}10 \\
(59 \%)\end{array}$ & $\begin{array}{c}5 \\
(30 \%)\end{array}$ & $\begin{array}{c}2 \\
(12 \%)\end{array}$ & $\begin{array}{c}0 \\
(0 \%)\end{array}$ & $\begin{array}{c}17 \\
(100 \%)\end{array}$ \\
\hline Total & $\begin{array}{c}90 \\
(25 \%)\end{array}$ & $\begin{array}{c}65 \\
(18 \%)\end{array}$ & $\begin{array}{c}204 \\
(57 \%)\end{array}$ & $\begin{array}{c}359 \\
(100 \%)\end{array}$ & $\begin{array}{c}120 \\
(33 \%)\end{array}$ & $\begin{array}{c}113 \\
(31 \%)\end{array}$ & $\begin{array}{c}91 \\
(25 \%)\end{array}$ & $\begin{array}{c}41 \\
(11 \%)\end{array}$ & $\begin{array}{c}365 \\
(100 \%)\end{array}$ \\
\hline
\end{tabular}

L'orientation des titres est majoritairement neutre (57\%), alors que le quart d'entre eux (25\%) sont défavorables et une plus faible proportion (18\%) s'avèrent favorables au requérant et/ou à la réduction du délai d'inadmissibilité à la libération conditionnelle. Le tableau laisse voir une tendance selon les régions. De la ColombieBritannique jusqu'au Québec, en effet, la proportion de titres défavorables diminue constamment. Ainsi, $42 \%$ des titres de la Colombie-Britannique ont une connotation négative, contre $26 \%$ pour les Prairies, $18 \%$ pour l'Ontario et seulement $9 \%$ pour le Québec. En Colombie-Britannique, le pourcentage élevé de titres défavorables s'explique par la requête de Clifford Olson (n= 63 titres), qui a fait l'objet d'une publicité fortement négative. Le requérant était représenté par lui-même, et les deux experts qu'il a convoqués à la barre des témoins (un psychiatre et une psychologue) ont affirmé qu'il était un pur psychopathe et que si un jour il allait être libéré, les probabilités à ce qu'il 
tue encore seraient très élevées. Durant la requête, d'autre part, Clifford Olson a déclaré qu'il savait très bien qu'il ne pourrait jamais accéder à une libération conditionnelle, mais qu'il faisait néanmoins preuve de bon caractère (l'un des critères pour décider s'il y a lieu de réduire le délai d'inadmissibilité à la libération conditionnelle (art. 745.63 du C.cr.)) en offrant une aide éventuelle aux autorités policières à retrouver des centaines de corps d'autres victimes qu'il aurait présumément tuées. Tous ces éléments, ainsi que d'autres (notamment les propos incendiaires de la part des proches des victimes), ont donné un ton majoritairement négatif aux titres et aux articles consacrés à la requête de Clifford Olson. Pour donner une idée de la connotation générale de la requête, les propos du juge au moment de laisser le jury délibérer, s'avèrent éloquents: "'Now I think all of us have had enough of this nasty business," he said, "and we will adjourn."' (Johnson et al., 1997: A3).

Contrairement à la Colombie-Britannique, les pourcentages de titres en défaveur des Prairies et de l'Ontario ne peuvent s'expliquer par une requête en particulier. Quant au Québec, la faible proportion de titres en défaveur est notamment due à la requête de Réal Chartrand ( $\mathrm{n}=45$ titres), où peu d'acteurs sociaux semblaient présents au tribunal pour s'opposer à la requête. D'après la lecture des articles, en effet, il n'y avait pas de proches de la victime qui assistaient à la requête, et seul un expert fut appelé à la barre des témoins pour s'opposer à la réduction éventuelle du délai d'inadmissibilité à la libération conditionnelle. En ce qui a trait aux provinces maritimes, finalement, une bonne partie des titres (35\%, ou 6 titres sur 17) s'avèrent défavorables.

À l'inverse, la proportion de titres favorables au requérant et/ou à la réduction du délai d'inadmissibilité à la libération conditionnelle augmente de façon constante en allant de l'Ouest jusqu'au Québec. En Colombie-Britannique, seulement 4\% des titres sont favorables, alors que c'est le cas pour 19\% des titres dans les Prairies, $21 \%$ en Ontario et $27 \%$ au Québec. Cette hausse de pourcentage (4\% à 27\%, donc 23\%) n'est toutefois pas aussi prononcée que la baisse de pourcentage présentée au niveau des titres 
défavorables (42\% à 9\%, donc 33\%). En Colombie-Britannique, le peu de titres favorables est grandement attribuable à la couverture journalistique négative de la requête de Clifford Olson. Au Québec, la requête de Réal Chartrand explique notamment la proportion importante de titres favorables. Effectivement, la quasi-totalité des témoins ayant comparu appuyaient la requête de Réal Chartrand, et ces déclarations favorables se reflétaient aussi bien dans les titres que dans les articles.

En ce qui a trait à l'orientation des articles, il faut tout d'abord remarquer que seulement un tiers d'entre eux (33\%) attribuent une connotation négative au requérant et/ou à la réduction du délai à l'inadmissibilité à la libération conditionnelle. Ce résultat va à l'encontre de ce qui aurait pu être déduit à partir des remarques anecdotiques affirmant que la révision judiciaire faisait l'objet d'une couverture médiatique négative (Roberts, 1994a, 1994b, 1995, 1997). Bien qu'il y ait une proportion non-négligeable d'articles défavorables, il reste néanmoins que cette orientation ne s'applique pas à la majorité de l'échantillon. De plus, une proportion presqu'égale d'articles (31\%) sont favorables, alors qu'un quart d'entre eux (25\%) sont équilibrés.

L'orientation des articles suit la même tendance que celle associée aux titres. En effet, 58\% des articles de la Colombie-Britannique se montrent en défaveur, comparativement à 35\% pour les Prairies, 16\% pour l'Ontario et 10\% pour le Québec. La Colombie-Britannique et les provinces maritimes (59\% ou 10 articles sur 17) représentent les seules régions où plus de la moitié des articles sont défavorables au requérant et/ou à la réduction du délai d'inadmissibilité à la libération conditionnelle. À l'opposé, le Québec constitue la seule région où plus de la moitié des articles sont favorables (53\%). Suivent ensuite, en ordre décroissant, l'Ontario (32\%), les provinces maritimes (29\%), les Prairies (27\%) et la Colombie-Britannique (18\%). Ici encore, la différence de pourcentage entre le Québec et la Colombie-Britannique demeure plus grande au niveau des articles en défaveur (58\% à 10\%, donc 48\%) qu'au niveau des articles en faveur (de 18\% à 53\%, donc 35\%). 
La répartition des articles équilibrés varie également selon la région du Canada. Bien que les Prairies (32\%) et l'Ontario (37\%) détiennent une proportion semblable d'articles équilibrés, le Québec en possède une moins grande proportion (21\%) alors que la Colombie-Britannique (10\%) et les provinces maritimes ( $12 \%$ ou 2 articles sur 17 ) se démarquent nettement des autres régions étant donné le peu d'articles équilibrés ayant été rédigés.

Comment expliquer les différences qui séparent l'est de l'ouest du Canada? Au Québec, comme il a été mentionné précédemment, la requête de Réal Chartrand n'a pas fait l'objet de litige. En effet, la quasi-totalité des individus retrouvés dans les articles témoignaient en faveur du requérant, sans qu'il n'y ait de réelle opposition à ce que la requête soit acceptée. D'autre part, aucun proche de victime ne fut cité ou mentionné dans les articles traitant des requêtes du Québec. Finalement, une collègue de travail a précisé qu'au Québec, la Couronne tend à s'opposer moins souvent aux requêtes qu'ailleurs au Canada ${ }^{13}$. L'ensemble de ces facteurs pourrait donc expliquer la faible proportion de titres et d'articles défavorables dans cette région.

Dans les autres régions, par contre (particulièrement dans les Prairies et en Colombie-Britannique), plusieurs commentaires de proches des victimes, ainsi que de membres de groupe de pression s'opposant à la requête, étaient rapportés dans les articles. Ces propos donnaient inévitablement un ton négatif à l'orientation de l'article. D'autre part, la présence-même de ces opposants a pu inciter les procureurs de la Couronne à s'opposer plus vigoureusement aux requêtes et ce, en contre-interrogeant davantage le requérant et les individus venus témoigner en sa faveur. À ces moments, il y aurait plus de chances de retrouver un contenu qui viendrait nuire au requérant et à sa

\footnotetext{
${ }^{13}$ La collègue de travail a obtenu cette information après avoir interviewé plusieurs procureurs de la Couronne ayant été affectés à ces requêtes.
} 
chance d'avoir droit à une réduction du délai d'inadmissibilité à la libération conditionnelle.

\subsubsection{2 - L'orientation des récits secondaires}

L'orientation des articles dépend donc grandement des événements qui se sont déroulés lors des requêtes, ainsi que des individus qui s'y sont présentés pour témoigner ou s'affirmer. Bien que les reporters puissent disposer d'une certaine marge de manoeuvre pour rapporter tel événement ou tel témoignage plutôt qu'un autre, cette marge de manoeuvre demeure toutefois limitée. Dans les récits secondaires (éditoriaux, chroniques, dossiers et lettres aux éditeurs), par contre, l'auteur de l'article peut émettre son opinion ouvertement. À propos des récits secondaires concernant la révision judiciaire, Roberts (1997) rapporte que: "L'article 745 est peut-être devenu le plus controversé du Code criminel. Il a donné lieu à d'innombrables éditoriaux et lettres aux rédacteurs, de même qu'à une couverture négative abondante dans les médias" (4). Ces éditoriaux et lettres d'opinion sont-ils majoritairement négatifs, comme le prétend l'auteur au sujet de la couverture médiatique en général? Pour vérifier cette hypothèse, les récits secondaires de la présente recherche ont été examinés selon leur orientation, et ils sont exposés au tableau XIV:

Tableau XIV:

Orientation de l'article selon les types de récit secondaire

\begin{tabular}{cccccc}
\hline $\begin{array}{c}\text { Type de récit } \\
\text { secondaire }\end{array}$ & Défavorable & Favorable & Neutre & Équilibré & Total \\
\hline Dossier & 2 & 2 & 1 & 1 & 6 \\
Lettre d'opinion & 8 & 2 & 3 & 0 & 13 \\
Éditorial & 0 & 6 & 1 & 0 & 7 \\
Chronique & 6 & 4 & 3 & 1 & 14 \\
Total & 16 & 14 & 8 & 2 & 40 \\
\hline
\end{tabular}


Sur les quarante récits secondaires échantillonnés, seize d'entre eux s'avèrent défavorables au requérant et/ou à la révision judiciaire, alors qu'un nombre presqu'égal d'articles $(\mathrm{n}=14)$ sont favorables. Les huit articles neutres proviennent majoritairement de la requête de Clifford Olson, où chroniqueurs et lecteurs de journaux faisaient part de leurs commentaires au sujet de l'intensité de la couverture journalistique consacrée à cette requête, sans toutefois se prononcer directement au sujet du requérant ou de la réduction du délai d'inadmissibilité à la libération conditionnelle.

L'orientation des récits secondaires varie selon leur type. Les dossiers sont répartis équitablement, deux d'entre eux étant défavorables et deux autres étant favorables. Les chroniques sont également divisées de façon assez équitable puisque sur un total de quatorze, six d'entre elles sont défavorables alors que quatre s'avèrent favorables. Toutefois, les lettres d'opinion sont majoritairement défavorables (8 lettres sur 13), peu d'entre elles étant favorables (2 sur 13). À l'inverse, la quasi-totalité des éditoriaux (6 sur 7) sont favorables à la révision judiciaire, le dernier éditorial se montrant neutre à ce sujet. Donc, en passant du lecteur au chroniqueur, et du chroniqueur à l'éditeur, la proportion de récits secondaires favorables augmente constamment. En discutant en profondeur des enjeux rattachés à la révision judiciaire, les éditoriaux considèrent donc que cette disposition légale s'avère nécessaire au bon fonctionnement du système de justice.

Bien que les éditoriaux aient l'avantage d'aller au-delà des faits en élaborant sur les implications rattachées à un sujet en particulier, ils sont toutefois moins souvent lus que les récits primaires. Une enquête menée par Statistique Canada (1997) révèle effectivement que 89\% des Canadiens âgés de 16 ans et plus lisent les pages des journaux consacrées à l'actualité locale et régionale (ce qui correspond aux récits primaires de la présente recherche), alors que ce pourcentage baisse à $60 \%$ pour les pages réservées aux éditoriaux. Donc, bien que les éditoriaux appuient la légitimité de 
la révision judiciaire, leur impact au niveau de l'opinion publique s'avère limité étant donné leur faible nombre et la proportion moindre de lecteurs qui les lisent.

\subsubsection{3 - L'orientation des titres et des articles selon les types de quotidien}

L'orientation des titres et des articles pourrait également varier selon le type de quotidiens ayant publié la nouvelle. Comme il a été vu précédemment, les proches des victimes et les procureurs de la Couronne sont plus souvent retenus comme source dominante dans les journaux tabloïds, favorisant ainsi la dimension de la loi et l'ordre au sujet de la révision judiciaire. Conséquemment, ces sources dominantes devraient influencer l'orientation des titres et des articles par rapport au requérant et/ou à la réduction du délai d'inadmissibilité à la libération conditionnelle. Le tableau XV présente la relation existant entre le type de quotidien et l'orientation des titres et des articles:

\section{Tableau XV:}

Orientation du titre et de l'article selon les types de quotidien de la Colombie-Britannique et du Québec

\begin{tabular}{|c|c|c|c|c|c|c|c|c|c|}
\hline \multirow{2}{*}{$\begin{array}{c}\text { Type } \\
\text { de quo- } \\
\text { tidien }\end{array}$} & \multicolumn{4}{|c|}{ Orientation du titre } & \multicolumn{5}{|c|}{ Orientation de l'article } \\
\hline & $\begin{array}{l}\text { Défav. } \\
\text { (\% r.) }\end{array}$ & $\begin{array}{c}\text { Faveur } \\
\text { (\% r.) }\end{array}$ & $\begin{array}{c}\text { Neutre } \\
\text { (\% r.) }\end{array}$ & $\begin{array}{l}\text { Total } \\
\text { (\% r.) }\end{array}$ & $\begin{array}{l}\text { Défav. } \\
\text { (\% r.) }\end{array}$ & $\begin{array}{c}\text { Faveur } \\
\text { (\% r.) }\end{array}$ & $\begin{array}{l}\text { Équil. } \\
\text { (\% r.) }\end{array}$ & $\begin{array}{c}\text { Neutre } \\
\text { (\% r.) }\end{array}$ & $\begin{array}{l}\text { Total } \\
\text { (\% r.) }\end{array}$ \\
\hline $\begin{array}{l}\text { Tabloid } \\
\text { (The } \\
\text { Prov.) }\end{array}$ & $\begin{array}{c}10 \\
(46 \%)\end{array}$ & $\begin{array}{c}0 \\
(0 \%)\end{array}$ & $\begin{array}{c}12 \\
(55 \%)\end{array}$ & $\begin{array}{c}22 \\
(100 \%)\end{array}$ & $\begin{array}{c}18 \\
(82 \%)\end{array}$ & $\begin{array}{c}1 \\
(5 \%)\end{array}$ & $\begin{array}{c}2 \\
(9 \%)\end{array}$ & $\begin{array}{c}1 \\
(5 \%)\end{array}$ & $\begin{array}{c}22 \\
(100 \%)\end{array}$ \\
\hline $\begin{array}{l}\text { Gr. for. } \\
\text { (Van. } \\
\text { Sun.) }\end{array}$ & $\begin{array}{c}15 \\
(41 \%)\end{array}$ & $\begin{array}{c}3 \\
(8 \%)\end{array}$ & $\begin{array}{c}19 \\
(51 \%)\end{array}$ & $\begin{array}{c}37 \\
(100 \%)\end{array}$ & $\begin{array}{c}18 \\
(47 \%)\end{array}$ & $\begin{array}{c}7 \\
(19 \%)\end{array}$ & $\begin{array}{c}4 \\
(11 \%)\end{array}$ & $\begin{array}{c}8 \\
(22 \%)\end{array}$ & $\begin{array}{c}37 \\
(100 \%)\end{array}$ \\
\hline $\begin{array}{l}\text { Tabloid } \\
\text { (J. de } \\
\text { Mont.) }\end{array}$ & $\begin{array}{c}3 \\
(11 \%)\end{array}$ & $\begin{array}{c}4 \\
(14 \%)\end{array}$ & $\begin{array}{c}21 \\
(75 \%)\end{array}$ & $\begin{array}{c}28 \\
(100 \%)\end{array}$ & $\begin{array}{c}7 \\
(25 \%)\end{array}$ & $\begin{array}{c}9 \\
(32 \%)\end{array}$ & $\begin{array}{c}9 \\
(32 \%)\end{array}$ & $\begin{array}{c}3 \\
(11 \%)\end{array}$ & $\begin{array}{c}28 \\
(100 \%)\end{array}$ \\
\hline $\begin{array}{l}\text { Gr. for. } \\
\text { (La } \\
\text { Presse) }\end{array}$ & $\begin{array}{c}2 \\
(8 \%)\end{array}$ & $\begin{array}{c}10 \\
(42 \%)\end{array}$ & $\begin{array}{c}12 \\
(50 \%)\end{array}$ & $\begin{array}{c}24 \\
(100 \%)\end{array}$ & $\begin{array}{c}0 \\
(0 \%)\end{array}$ & $\begin{array}{c}16 \\
(67 \%)\end{array}$ & $\begin{array}{c}4 \\
(17 \%)\end{array}$ & $\begin{array}{c}4 \\
(17 \%)\end{array}$ & $\begin{array}{c}24 \\
(100 \%)\end{array}$ \\
\hline $\begin{array}{l}\text { Gr. for. } \\
\text { (The } \\
\text { Gazet.) }\end{array}$ & $\begin{array}{c}1 \\
(7 \%)\end{array}$ & $\begin{array}{c}5 \\
(33 \%)\end{array}$ & $\begin{array}{c}9 \\
(60 \%)\end{array}$ & $\begin{array}{c}15 \\
(100 \%)\end{array}$ & $\begin{array}{c}0 \\
(0 \%)\end{array}$ & $\begin{array}{c}11 \\
(73 \%)\end{array}$ & $\begin{array}{c}2 \\
(13 \%)\end{array}$ & $\begin{array}{c}2 \\
(13 \%)\end{array}$ & $\begin{array}{c}15 \\
(100 \%)\end{array}$ \\
\hline Total & $\begin{array}{c}31 \\
(25 \%)\end{array}$ & $\begin{array}{c}22 \\
(18 \%)\end{array}$ & $\begin{array}{c}73 \\
(58 \%)\end{array}$ & $\begin{array}{c}126 \\
(100 \%)\end{array}$ & $\begin{array}{c}43 \\
(34 \%)\end{array}$ & $\begin{array}{c}44 \\
(35 \%)\end{array}$ & $\begin{array}{c}21 \\
(17 \%)\end{array}$ & $\begin{array}{c}18 \\
(14 \%)\end{array}$ & $\begin{array}{c}126 \\
(100 \%)\end{array}$ \\
\hline
\end{tabular}


Au niveau des titres, il n'y a pas de différence notable entre les deux types de journaux de la Colombie-Britannique. Au Québec, par contre, le Journal de Montréal se démarque des deux journaux de type grand format car il n'a publié que quatre titres favorables sur une possibilité de vingt-huit. Cette proportion diverge sensiblement de celles de La Presse (10 sur 24) et The Gazette (5 sur 15). Toutefois, le faible nombre de titres favorables du journal tabloid ne se traduit pas par une hausse de titres défavorables. Au lieu, le Journal de Montréal détient une proportion plus élevée de titres neutres (21 sur 28) que La Presse (12 sur 24) et The Gazette (9 sur 15). Il est également intéressant de constater qu'il n'y a pas de différence remarquable entre le journal grand format francophone et son compétiteur anglophone (The Gazette).

En ce qui a trait aux articles, les journaux tabloïds de la Colombie-Britannique et du Québec ont publié une plus grande proportion d'articles défavorables que leurs compétiteurs respectifs. Le tabloïd The Province, par exemple, détient dix-huit articles d'orientation négative sur une possibilité de vingt-deux, alors que c'est le cas de dix-huit articles sur trente-sept pour le grand format Vancouver Sun. Au Québec, le Journal de Montréal a publié sept articles défavorables sur 28, alors que les deux journaux de type grand format n'ent ont publié aucun. À l'inverse, les journaux de type grand format ont rédigé davantage d'articles favorables. Le quotidien grand format de la ColombieBritannique en a rédigé sept sur trente-sept, contre un sur vingt-deux pour son compétiteur de type tabloïd. Au Québec, la majorité des articles de La Presse (16 sur 24) et The Gazette (11 sur 15) s'avérent favorables, contre neuf articles favorables sur vingt-huit pour le Journal de Montréal. Il faut toutefois noter que ce dernier a rédigé davantage d'articles équilibrés (9 sur 28) que La Presse (4 sur 24) et The Gazette (2 sur 15). Finalement, comme ce fut le cas au niveau des titres, les articles des journaux grand format francophone et anglophone du Québec sont répartis de façon presqu'identique à travers les quatre catégories d'orientation. 
3.2.2.4 - L'orientation des titres et des articles selon les caractéristiques liées aux homicides commis par les requérants

L'orientation des titres et des articles pourrait également varier selon les caractéristiques liées aux homicides commis par les requérants. L'ensemble des requérants étant des hommes, peuvent-ils faire l'objet d'une couverture journalistique différente selon qu'ils aient tué un homme ou une femme? Pour répondre à cette question, ainsi qu'à d'autres, le tableau XVI croise les caractéristiques liées à ces homicides avec l'orientation des titres et des articles.

L'orientation des titres varie de façon importante avec une seule variable, soit le nombre de victimes tuées par le requérant. En effet, 34\% des titres concernant les meurtriers multiples sont défavorables, contre 22\% des titres rattachés à ceux n'ayant tué qu'une victime. De plus, seulement 7\% des titres rattachés aux meurtriers multiples sont favorables, contre 22\% pour les requérants n'ayant tué qu'une personne. Il faut toutefois noter que ces résultats sont largement tributaires de la requête de Clifford Olson, qui fut médiatisée de façon négative aussi bien dans les titres que dans les articles.

Au niveau de l'orientation des articles, une remarque s'impose avant tout: bien que l'orientation des articles semble varier selon le type de condamnation du requérant, le faible nombre d'articles répertoriés au sujet des requérants ayant été condamnés pour meurtre au deuxième degré $(n=16)$, pour meurtre capital $(n=15)$ et pour meurtre noncapital ( $\mathrm{n}=16)$ limite la validité d'interprétation des résultats. Cette remarque s'applique également à la relation entre l'orientation des articles et le type d'homicide commis par le requérant, où peu d'articles traitant d'homicides passionnels ou familiaux furent échantillonnés $(\mathrm{n}=21)$. Pour cette dernière relation, par contre, une nette tendance 
semble se dessiner: les requérants ayant commis un homicide familial ou passionnel font davantage l'objet d'une couverture journalistique positive (62\% ou 13 articles sur 21) que

Tableau XVI:

Orientation du titre et de l'article selon les caractéristiques liées aux homicides

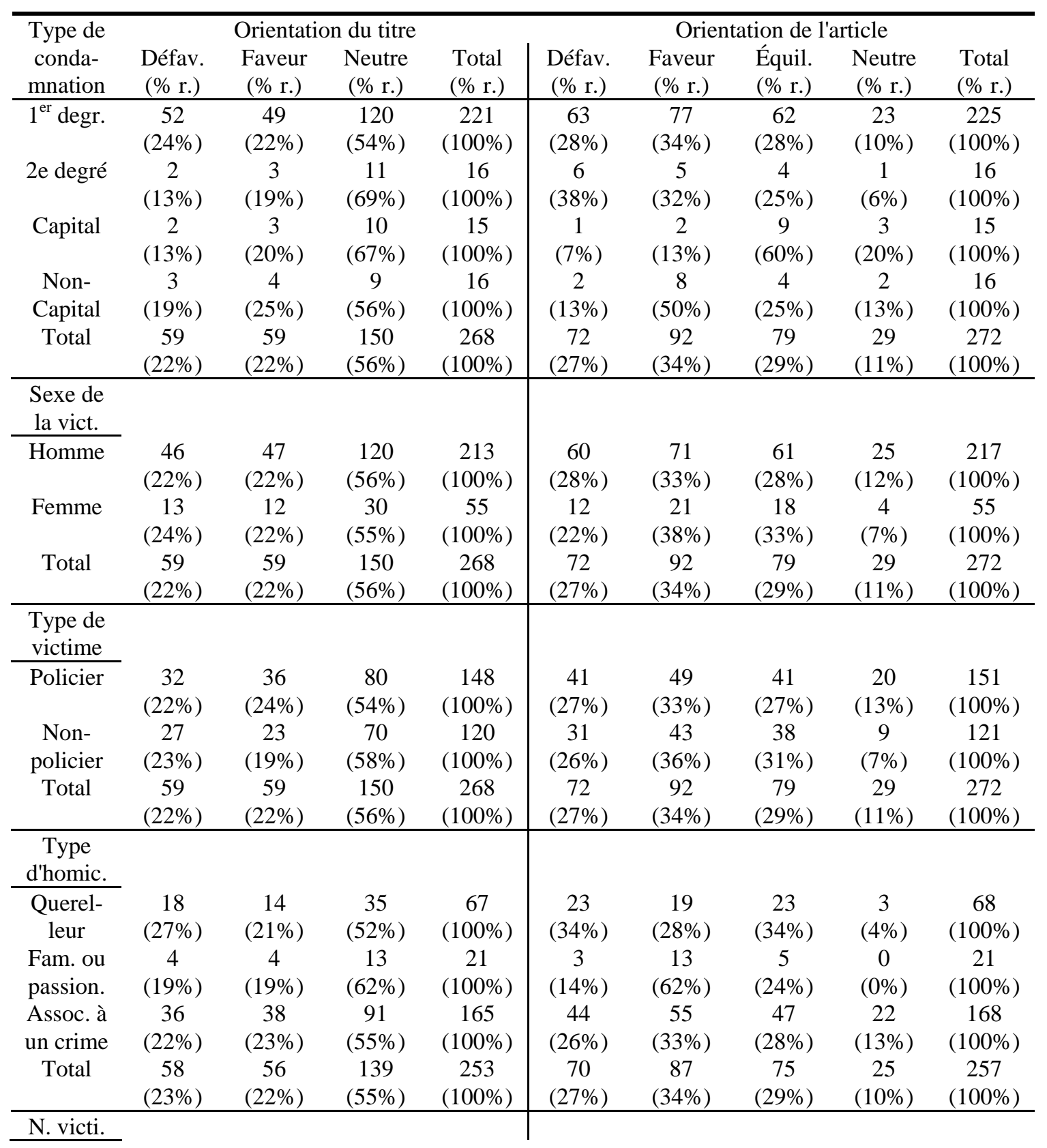




\begin{tabular}{ccccc|ccccc} 
Une & 59 & 59 & 150 & 268 & 72 & 92 & 79 & 29 & 272 \\
& $(22 \%)$ & $(22 \%)$ & $(56 \%)$ & $(100 \%)$ & $(27 \%)$ & $(34 \%)$ & $(29 \%)$ & $(11 \%)$ & $(100 \%)$ \\
Deux et & 31 & 6 & 54 & 91 & 48 & 21 & 12 & 12 & 93 \\
plus & $(34 \%)$ & $(7 \%)$ & $(59 \%)$ & $(100 \%)$ & $(52 \%)$ & $(23 \%)$ & $(13 \%)$ & $(13 \%)$ & $(100 \%)$ \\
Total & 90 & 65 & 204 & 359 & 120 & 113 & 91 & 41 & 365 \\
& $(25 \%)$ & $(18 \%)$ & $(57 \%)$ & $(100 \%)$ & $(33 \%)$ & $(31 \%)$ & $(25 \%)$ & $(11 \%)$ & $(100 \%)$ \\
\hline
\end{tabular}

ceux ayant commis un homicide querelleur (28\%) ou associé à un autre crime (33\%). Aussi, les auteurs de meurtre passionnel ou familial font moins souvent l'objet d'une couverture journalistique négative (14\% ou 3 articles sur 21) que ceux ayant commis un meurtre querelleur (34\%) ou associé à un autre crime (26\%). Selon les dires des avocats de la défense, des agents correctionnels et des experts cités ou mentionnés dans les articles, la majorité des requérants ayant commis un homicide passionnel ou familial ont fait preuve d'un comportement exemplaire dans les pénitenciers. Ces propos se sont donc reflétés dans l'orientation des articles à leur sujet. Finalement, l'orientation des articles varie également en fonction du nombre de victimes tuées par le requérant. En effet, 52\% des articles concernant les meurtriers multiples sont défavorables, contre 27\% pour les meurtriers n'ayant commis qu'un meurtre. À l'inverse, 23\% des articles traitant de meurtriers multiples s'avèrent favorables, contre 34\% pour les meurtriers n'ayant tué qu'une victime. Encore une fois, ces résultats sont dûs en grande partie aux articles consacrés à la requête de Clifford Olson.

\subsection{3 - L'exactitude des articles}

Plusieurs auteurs ont affirmé que les journalistes manquent d'expérience et ne possédent pas une connaissance suffisante des procédures judiciaires (Drechsel, 1978, 1983; Ericson et al. 1989; Grabosky et Wilson, 1989; Lotz, 1991). Selon certains acteurs du champ judiciaire, ces lacunes expliqueraient la quantité importante de faits inexacts retrouvés dans les articles de quotidiens traitant de procès en droit criminel (Ericson et al., 1989; Lotz, 1991). Combinés avec la complexité des procès (Ericson et al., 1989; Lotz, 1991) et la contrainte organisationnelle des maisons de presse qui vise à simplifier les événements judiciaires (Ericson et al., 1987, 1989), ces facteurs contribueraient à faire apparaître des fait erronnés ou inexacts dans les chroniques 
judiciaires. Et comme de fait, il a été rapporté que les médias n'explicitaient pas convenablement le processus de la révision judiciaire (O'Reilly-Fleming, 1991; Roberts, 1994a). La présente section s'affairera donc à évaluer l'exactitude des propos rapportés dans les articles au sujet de la nature et du fonctionnement de la révision judiciaire.

\subsubsection{1 - L'exactitude des articles selon les régions du Canada}

Le tableau XVII décrit l'exactitude des articles selon les différentes régions du Canada. Avant d'interpréter ce tableau, il faut préciser que certains articles ( $\mathrm{n}=22)$ ont été exclus des analyses car ils n'abordaient pas l'aspect légal de la révision judiciaire. Contrairement aux faits énoncés dans la littérature, peu d'articles (12\%) ont rapporté des propos inexacts au sujet de la révision judiciaire. En effet, la très grande majorité d'entre eux (88\%) ont traité de la révision judiciaire de façon exacte et ce, malgré la relative complexité de l'article 745.6 du Code criminel.

Tableau XVII:

Exactitude de l'article selon les régions du Canada

\begin{tabular}{cccc}
\hline Région & Exact (\% rangée) & $\begin{array}{c}\text { Exactitude de l'article } \\
\text { Inexact (\% rangée) }\end{array}$ & Total (\% rangée) \\
\hline C.-B. & $47(77 \%)$ & $14(23 \%)$ & $61(100 \%)$ \\
Prairies & $131(90 \%)$ & $15(10 \%)$ & $146(100 \%)$ \\
Ontario & $53(93 \%)$ & $4(7 \%)$ & $57(100 \%)$ \\
Québec & $55(87 \%)$ & $8(13 \%)$ & $63(100 \%)$ \\
Maritimes & $15(94 \%)$ & $1(6 \%)$ & $16(100 \%)$ \\
Total & $301(88 \%)$ & $42(12 \%)$ & $343(100 \%)$ \\
\hline
\end{tabular}

La région ayant publié la plus grande portion de faits erronnés est la ColombieBritannique (23\% ou 14 articles sur 61). La majorité de ces articles (11 articles sur 14) étaient rattachés à la requête de Clifford Olson, et certains d'entre eux laissaient l'impression que si le jury acceptait favorablement la requête, le requérant obtenait 
immédiatement sa libération conditionnelle. À l'autre extrémité, les régions ayant publié le moins d'articles inexacts sont l'Ontario ( $7 \%$ ou 4 articles sur 57 ) et les provinces maritimes (6\% ou 1 article sur 16 ).

Dans certains cas, il arrivait que l'explication de la révision judiciaire soit identique pour des articles traitant d'une même requête. Par exemple, l'explication du processus législatif paraissait sur un premier article de quotidien, et l'article publié le lendemain reprenait cette explication mot pour mot. Si les propos rapportés dans le premier article s'avéraient inexacts, ils l'étaient donc aussi pour les articles subséquents. Cette reproduction d'inexactitudes s'effectuait également lorsqu'un quotidien recopiait partiellement ou complètement un article inexact provenant d'un autre quotidien ou de la Presse canadienne. De cette façon, "inaccuracies are perpetuated because second-level reproducers of knowledge typically do not bother to check what their respected peers have said appears to be the case" (Ericson et al., 1987: 268).

\subsubsection{2 - L'exactitude des articles selon les types de quotidien}

Étant donné que les journaux tabloïds mettent l'accent sur le côté émotionnel des événements, alors que les journaux de type grand format tendent davantage à expliquer la raison d'être de ces événements (Ericson et al., 1991), il pourrait y avoir une différence d'exactitude des articles selon le type de quotidien. En effet, les journaux tabloïds pourraient accorder une moins grande importance à l'explication de la révision judiciaire, rendant ainsi leurs articles plus susceptibles d'être inexacts que ceux provenant des quotidiens de type grand format. Le tableau XVIII vérifie la validité de cette proposition.

Contrairement à ce qui a été postulé, ce sont les journaux de type grand format qui ont publié le plus d'articles inexacts. Le tiers des articles du Vancouver Sun (10 
articles sur 30) s'avèrent effectivement inexacts, contre seulement deux articles inexacts sur quinze pour son rival tabloïd. Au Québec, La Presse a rapporté des propos erronés

Tableau XVIII:

Exactitude de l'article selon les types de quotidien de la Colombie-Britannique et du Québec

\begin{tabular}{cccc}
\hline Type de quotidien & Exact & $\begin{array}{c}\text { Exactitude de l'article } \\
\text { Inexact }\end{array}$ & Total \\
\hline Tabloid (The Province) & 15 & 2 & 17 \\
Gr. form. (Van. Sun) & 20 & 10 & 30 \\
Tabloid (Jou. de Mont.) & 21 & 2 & 23 \\
Gr. form. (La Presse) & 17 & 5 & 22 \\
Gr. form. (TheGazet.) & 14 & 1 & 15 \\
Total & 87 & 20 & 107 \\
\hline
\end{tabular}

dans cinq de ses vingt-deux articles, alors que c'est le cas de deux articles sur vingt et un pour le Journal de Montréal. The Gazette, par contre, contraste avec les autres quotidiens de type grand format car ce quotidien n'a publié qu'un article inexact sur une possibilité de quinze. Donc, bien que les tabloïds utilisent davantage les proches des victimes comme source dominante, et bien qu'ils détiennent une quantité importante d'articles défavorables au sujet du requérant et/ou de la réduction du délai d'inadmissibilité à la libération conditionnelle, il reste néanmoins que la quasi-totalité des articles qu'ils ont publié expliquent convenablement le processus de révision judiciaire.

L'exactitude des articles ne diffère pas selon le type de récit secondaire ou selon les caractéristiques liées aux homicides des requérants (tableaux B1 et B2 dans l'annexe B).

\section{3 - Les facteurs associés à l'intensité de la couverture journalistique des requêtes}


Parmi les requêtes qui ont été publiées, certaines ont fait l'objet d'une faible couverture journalistique alors que d'autres ont paru dans un nombre considérable d'articles. Bien qu'il ait été mentionné que les procès qui ont le plus de chance d'être publiés soient ceux impliquant la perpétration de meurtres ou de tentatives de meurtre (Lotz, 1991), ceux où des personnages publics ou des professionnels ayant du pouvoir sont accusés (Ericson et al., 1989; Lotz, 1991; Rosenfeld, 1988; Tremblay, 1988), ainsi que ceux impliquant une sentence d'emprisonnement (Graber, 1980; Roberts, 1988a) ou une sentence extrêmement légère selon l'avis des journalistes (Canada, 1987a; Rosenfeld, 1988), aucune étude ne s'est attardée à identifier les facteurs associés à l'intensité de publication (que ce soit le nombre d'articles, leur position dans le journal, l'espace qu'ils occupent, etc.) des procès.

Toutefois, certains auteurs ont étudié les facteurs qui prédisent l'intensité de la couverture journalistique de crimes ou d'homicides perpétrés, et ils en arrivent presque tous à la même conclusion: un nombre croissant de victimes impliquées dans les événements criminels constitue le meilleur prédicteur d'une couverture journalistique intense (Chermak, 1998a; Johnstone et al., 1994; Wilbanks, 1994). De leur côté, Pritchard et Hughes (1997) ont découvert que les homicides impliquant une femme en tant que victime faisaient l'objet d'une couverture journalistique plus intense.

Dans le cadre de la présente recherche, certaines caractéristiques associées aux requêtes publiées ont été croisées avec leur intensité de publication ${ }^{14}$. Le tableau XIX fait état de ces résultats. Il y a une relation significative entre le type de victime tué par le requérant et l'intensité de publication de la requête. En effet, 89\% des requêtes impliquant le meurtre d'un policier (ou 8 sur 9) ont fait l'objet d'une forte couverture journalistique, contre 33\% des requêtes n'impliquant pas ce type de victime. Il y a

\footnotetext{
14 Pour les fins de la présente recherche, les requêtes ayant fait l'objet de 5 articles et plus correspondent à des requêtes où la couverture journalistique s'avére intense. Bien que les résultats de cette variable puissent être tributaires du nombre de quotidiens échantillonnés dans chaque province, nous considérons que la catégorie " 5 articles et plus" constitue un juste milieu pour l'ensemble des requêtes publiées.
} 
également une relation significative entre la présence d'opposants à la révision judiciaire (groupe de pression, policiers, politiciens) et l'intensité de publication des requêtes. Ici,

Tableau XIX:

Nombre d'articles consacrés aux requêtes selon leurs caractéristiques

\begin{tabular}{|c|c|c|c|c|}
\hline \multirow{2}{*}{$\begin{array}{c}\text { Type de } \\
\text { condamnation }\end{array}$} & \multicolumn{3}{|c|}{ Nombre d'articles consacrés à la requête } & \multirow{2}{*}{$\begin{array}{l}\text { Test du } \\
\text { chi-carré }\end{array}$} \\
\hline & 1 à 4 (\% rang.) & 5 et plus (\% rang.) & Total (\% rang.) & \\
\hline $1^{\mathrm{er}}$ degré & $14(52 \%)$ & $13(48 \%)$ & $27(100 \%)$ & \\
\hline 2e degré & $1(33 \%)$ & $2(67 \%)$ & $3(100 \%)$ & \\
\hline Capital & $1(50 \%)$ & $1(50 \%)$ & $2(100 \%)$ & \\
\hline Non-capital & $3(75 \%)$ & $1(25 \%)$ & $4(100 \%)$ & \\
\hline Total & $19(53 \%)$ & $17(47 \%)$ & $36(100 \%)$ & 1.2 \\
\hline \multicolumn{5}{|l|}{ Sexe de la victime } \\
\hline Homme & $11(44 \%)$ & $14(56 \%)$ & $25(100 \%)$ & \\
\hline Femme & $8(73 \%)$ & $3(27 \%)$ & $11(100 \%)$ & \\
\hline Total & $19(53 \%)$ & $17(47 \%)$ & $36(100 \%)$ & 2.5 \\
\hline \multicolumn{5}{|l|}{ Type de victime } \\
\hline Policier & $1(11 \%)$ & 8 (89\%) & $9(100 \%)$ & \\
\hline Non-policier & $18(67 \%)$ & 9 (33\%) & 27 (100\%) & \\
\hline Total & $19(53 \%)$ & $17(47 \%)$ & $36(100 \%)$ & $8.4 * *$ \\
\hline \multicolumn{5}{|l|}{ Type d'homicide } \\
\hline Querelleur & $6(50 \%)$ & $6(50 \%)$ & $12(100 \%)$ & \\
\hline Familial ou passionnel & $4(80 \%)$ & $1(20 \%)$ & $5(100 \%)$ & \\
\hline Associé à un crime & 7 (47\%) & $8(53 \%)$ & 15 (100\%) & \\
\hline Total & 17 (53\%) & $15(47 \%)$ & 32 (100\%) & 1.7 \\
\hline \multicolumn{5}{|l|}{ Nombre de victimes } \\
\hline Une & 19 (53\%) & 17 (47\%) & $36(100 \%)$ & \\
\hline Deux et plus & 7 (64\%) & $4(36 \%)$ & $11(100 \%)$ & \\
\hline Total & $26(55 \%)$ & $21(45 \%)$ & $47(100 \%)$ & 0.4 \\
\hline \multicolumn{5}{|l|}{ Ordre chronologique } \\
\hline $1^{\text {ère }}$ à $3^{\mathrm{e}}$ requête & $8(44 \%)$ & $10(56 \%)$ & 18 (100\%) & \\
\hline 4e requête et plus & $18(62 \%)$ & $11(38 \%)$ & $29(100 \%)$ & \\
\hline Total & $26(55 \%)$ & $21(45 \%)$ & $47(100 \%)$ & 1.4 \\
\hline \multicolumn{5}{|l|}{ Présence d'opposants } \\
\hline Non & $25(68 \%)$ & $12(32 \%)$ & 37 (100\%) & \\
\hline Oui & $1(10 \%)$ & $9(90 \%)$ & $10(100 \%)$ & \\
\hline Total & $26(55 \%)$ & $21(45 \%)$ & 47 (100\%) & $10.6 * *$ \\
\hline
\end{tabular}

Note: ** Indique une relation significative à 0.01 . 
90\% des requêtes comprenant des opposants (ou 9 sur 10) ont fait l'objet d'une couverture journalistique intense, comparativement à seulement 32\% des requêtes n'incluant pas d'opposants. Incidemment, les requêtes impliquant le meurtre d'un policier ont souvent été accompagnées d'une présence d'opposants à l'article 745.6 du Code criminel:

\section{Tableau XX:}

Présence d'opposants lors de la requête selon les types de victime

\begin{tabular}{ccccc}
\hline Type de victime & \multicolumn{2}{c}{ Présence d'opposants lors de la requête } & Test du \\
& Non (\% rangée) & Oui (\% rangée) & Total (\% rangée) & chi-carré \\
\hline Policier & $4(44 \%)$ & $5(56 \%)$ & $9(100 \%)$ & \\
Autre & $23(85 \%)$ & $4(15 \%)$ & $27(100 \%)$ & \\
Total & $27(75 \%)$ & $9(25 \%)$ & $36(100 \%)$ & $6.0^{*}$ \\
\hline
\end{tabular}

Note: * Indique une relation significative à 0.05 .

Le tableau XX démontre effectivement que plus de la moitié des requêtes impliquant le meurtre d'un policier (5 sur 9) ont été accompagnées d'une présence d'opposants, contre seulement quatre requêtes sur un total de vingt-sept pour les requêtes où la victime du requérant n'était pas un policier.

\section{4 - Les facteurs associés à la réduction éventuelle du délai d'inadmissibilité à la libération conditionnelle}

La décision des jurys de réduire le délai d'inadmissibilité à la libération conditionnelle des requérants pourrait être associée à certaines variables de la présente étude. Comme il a été indiqué dans le premier chapitre, les procureurs de la Couronne veulent paraître intransigeants face aux criminels lorsque les procès qu'ils dirigent sont couverts par les médias (Pritchard, 1986). Ce constat pourrait également s'appliquer à la présente étude, où les procureurs de la Couronne s'opposeraient plus rigoureusement 
aux requêtes lorsque celles-ci feraient l'objet d'une couverture journalistique ${ }^{15}$. Conséquemment, ces requêtes auraient plus de chances de faire l'objet d'un refus de la part du jury.

Bien que l’objectif de la présente section soit d'analyser la relation existant entre la couverture journalistique des requêtes et la décision des jurys, d’autres facteurs légaux et extra-légaux seront également croisés avec la décision des jurys afin d’identifier d'éventuelles relations significatives. Au sujet des requêtes de révision judiciaire, Roberts (1994a) avançait notamment que "public antipathy will be particularly strong in cases of first degree murder in which the victim was a police officer" (7). Est-ce que cette antipathie se réflèterait dans la décision des jurys, eux-mêmes étant constitués par des membres du public? Ce facteur, ainsi que d’autres, sont croisés avec la décision des jurys de réduire ou non le délai d’inadmissibilité à la libération conditionnelle des requérants au tableau XXI.

Il y a une relation significative entre la parution des requêtes dans les quotidiens échantillonnés et la décision des jurys. Comme le tableau XXI le démontre, seulement 11\% des requêtes non-publiées ont été refusées par les jurys, comparativement à 33\% des requêtes ayant paru dans les journaux. Cette différence de pourcentage pourrait être expliquée par l'hypothèse exposée plus haut. En effet, il est possible que les procureurs de la Couronne s’opposent davantage aux requêtes lorsque des journalistes se présentent durant les audiences, résultant ainsi en un plus grand nombre de refus de la part des jurys à considérer une réduction du délai d’inadmissibilité à la libération conditionnelle.

Ces résultats peuvent toutefois être interprétés d'autres façons. Premièrement, il se pourrait que la présence des journalistes incite les jurys à prendre leur décision avec une plus grande prudence. Étant conscients que la requête fait l'objet d'une attention médiatique, ils pourraient opter pour la décision la plus conservatrice qui soit, c'est-à15 Pour tester cette hypothèse, seules les requêtes ayant fait l'objet d'une publication avant que la décision
des jurys soit rendue, furent retenues pour l'analyse. 
dire ne pas réduire le délai d'inadmissibilité à la libération conditionnelle du requérant. Puis, étant donné que les journalistes ont tendance à couvrir les événements les plus sensationnels (Morissette, 1991; Roberts, 1992), il se peut que la majorité des requêtes publiées impliquent des cas où les homicides commis par les requérants furent plus spectaculaires et plus horribles que les homicides associés aux requêtes qui ne furent pas

Tableau XXI:

Décision au sujet de la période d'inadmissibilité à la libération conditionnelle selon les caractéristiques liées aux requêtes

\begin{tabular}{|c|c|c|c|c|}
\hline Parution do la reguêto & \multicolumn{3}{|c|}{ Décision au sujet de la période d'inadm. à la lib. cond. } & \multirow{2}{*}{$\begin{array}{l}\text { Test du } \\
\text { chi-carré }\end{array}$} \\
\hline & & & & \\
\hline Non & $6(11 \%)$ & $50(89 \%)$ & $56(100 \%)$ & \\
\hline Oui & $12(33 \%)$ & $24(67 \%)$ & $36(100 \%)$ & \\
\hline Total & $18(20 \%)$ & $74(80 \%)$ & $92(100 \%)$ & $7.1 * *$ \\
\hline \multicolumn{5}{|l|}{ Nombre d'articles } \\
\hline 1 à 4 & $8(31 \%)$ & $18(69 \%)$ & $26(100 \%)$ & \\
\hline 5 et plus & $6(29 \%)$ & $15(71 \%)$ & $21(100 \%)$ & \\
\hline Total & $14(30 \%)$ & $33(70 \%)$ & $47(100 \%)$ & 0.0 \\
\hline \multicolumn{5}{|l|}{ Orientat. de la requête } \\
\hline Défaveur & $4(44 \%)$ & $5(56 \%)$ & $9(100 \%)$ & \\
\hline Faveur & $6(30 \%)$ & $14(70 \%)$ & $20(100 \%)$ & \\
\hline Équilibré & $2(40 \%)$ & $3(60 \%)$ & $5(100 \%)$ & \\
\hline Neutre & $0(0 \%)$ & $2(100 \%)$ & $2(100 \%)$ & \\
\hline Total & $12(33 \%)$ & $24(67 \%)$ & $36(100 \%)$ & 1.7 \\
\hline \multicolumn{5}{|l|}{ Type de condamnat. } \\
\hline $1^{\mathrm{er}}$ degré & $8(30 \%)$ & $19(70 \%)$ & 27 (100\%) & \\
\hline 2e degré & $0(0 \%)$ & $3(100 \%)$ & $3(100 \%)$ & \\
\hline Capital & $1(50 \%)$ & $1(50 \%)$ & $2(100 \%)$ & \\
\hline Non-capital & $0(0 \%)$ & $4(100 \%)$ & $4(100 \%)$ & \\
\hline Total & $9(25 \%)$ & $27(75 \%)$ & $36(100 \%)$ & 3.3 \\
\hline \multicolumn{5}{|l|}{ Sexe de la victime } \\
\hline Homme & $5(20 \%)$ & $20(80 \%)$ & $25(100 \%)$ & \\
\hline Femme & $4(36 \%)$ & $7(64 \%)$ & $11(100 \%)$ & \\
\hline Total & $9(25 \%)$ & $27(75 \%)$ & $36(100 \%)$ & 1.1 \\
\hline \multicolumn{5}{|l|}{ Type de victime } \\
\hline Policier & $3(33 \%)$ & $6(67 \%)$ & $9(100 \%)$ & \\
\hline Non-policier & $6(22 \%)$ & 21 (78\%) & $27(100 \%)$ & \\
\hline Total & $9(25 \%)$ & 27 (75\%) & $36(100 \%)$ & 0.4 \\
\hline \multicolumn{5}{|l|}{ Type d'homicide } \\
\hline Querelleur & $0(0 \%)$ & $12(100 \%)$ & $12(100 \%)$ & \\
\hline Familial ou passionnel & $3(60 \%)$ & $2(40 \%)$ & $5(100 \%)$ & \\
\hline Associé à un crime & $5(33 \%)$ & $10(67 \%)$ & $15(100 \%)$ & \\
\hline Total & $8(25 \%)$ & $24(75 \%)$ & $32(100 \%)$ & $7.8^{*}$ \\
\hline \multicolumn{5}{|l|}{ Nombre de victimes } \\
\hline Une & $9(25 \%)$ & 27 (75\%) & $36(100 \%)$ & \\
\hline Deux et plus & $5(46 \%)$ & $6(54 \%)$ & $11(100 \%)$ & \\
\hline Total & $14(30 \%)$ & $33(70 \%)$ & $47(100 \%)$ & 1.7 \\
\hline
\end{tabular}




\begin{tabular}{ccccc}
\cline { 1 - 1 } Ordre chronologique & & & & \\
\cline { 1 - 1 } $1^{\text {ère }}$ à 3e requête & & & & \\
4e requête et plus & $8(28 \%)$ & $12(67 \%)$ & $18(100 \%)$ & \\
Total & $14(30 \%)$ & $21(72 \%)$ & $29(100 \%)$ & \\
\cline { 1 - 1 } Présence d'opposants & & $33(70 \%)$ & $47(100 \%)$ & 0.2 \\
\hline Non & $9(24 \%)$ & $28(76 \%)$ & $37(100 \%)$ & \\
Oui & $5(50 \%)$ & $5(50 \%)$ & $10(100 \%)$ & \\
Total & $14(30 \%)$ & $33(70 \%)$ & $47(100 \%)$ & 2.5 \\
\hline
\end{tabular}

Note: **/* Indique une relation significative à 0.01 et 0.05 , respectivement.

publiées. Et puisque les jurys doivent notamment considérer la nature de l'infraction pour laquelle le requérant a été condamné afin de prendre leur décision (art.745.63(1) du C.cr.), il serait normal que les requérants ayant perpétré des homicides jugés plus graves aient moins de chances de se voir attribuer une réduction du délai d'inadmissibilité à la libération conditionnelle. La relation entre la couverture journalistique des requêtes et la décision des jurys serait donc artificielle.

D’autre part, il faut mentionner que la majorité des requêtes qui furent nonpubliées et acceptées par les jurys proviennent du Québec $(n=35)$, ce qui soulève d'autres interrogations par rapport à l'existence réelle d'une association entre les deux variables. En effet, l'existence de cette relation pourrait plutôt être tributaire de deux éléments distincts, soit la tendance des procureurs de la Couronne du Québec à ne pas s'opposer aux requêtes ${ }^{16}$, et l'effet de nouveauté des requêtes du Québec qui s'est estompé étant donné leur grand nombre ( $n=43)$.

Le tableau XXI démontre également que la décision des jurys varie de façon significative selon le type d'homicide commis par le requérant. Ainsi, la totalité des requêtes impliquant un homicide querelleur ont fait l'objet d'une réduction, alors que c'est le cas pour $67 \%$ des requêtes (10 sur 15) impliquant un homicide associé à un autre crime et pour $40 \%$ des requêtes ( 2 sur 5) impliquant des meurtres familiaux ou passionnels. Les réductions de délai des homicides querelleurs pourraient notamment

\footnotetext{
${ }^{16}$ Ces faits m'ont été rapportés par une collègue qui a interviewé des procureurs de la Couronne et des avocats de la défense ayant été affectés à de telles requêtes.
} 
être expliquées par le fait que ce type d'homicide résulte souvent d'une provocation de la part des victimes (Grandmaison, 1993), et les jurys ont pu considérer ce facteur atténuant dans leur décision. Pour leur part, le moins haut taux de réussite des requérants ayant commis un homicide associé à un autre crime pourrait être dû au fait que ce type d'homicide soit considéré plus grave par la population étant donné qu'il victimise un innocent ou un étranger (Daly et Wilson, 1988). Puis, contrairement à ce qui est rapporté dans la littérature (Cusson, 1987; Lundsgaarde, 1977), les homicides familiaux ou passionnels ne semblent pas considérés comme étant moins graves dans la présente étude. Cependant, le faible nombre de requêtes retrouvées dans cette catégorie limite l'interprétation que l'on peut faire de ces résultats.

L'intensité et l'orientation de la requête dans les quotidiens ne sont pas associées statistiquement avec la décision des jurys. Il en va de même pour le type de condamnation, ainsi que pour le sexe, le type, et le nombre de victimes impliquées dans les requêtes. Donc, les jurys ne se sont pas montrés particulièrement antipathiques envers les requérants ayant tué un policier. Ce résultat pourrait s'expliquer par le processus de filtrage des jurés potentiels qui consiste à leur demander s'ils ont ou non des préjugés envers le type de requérant qui fait une demande (ici, il s'agit de savoir si les jurés potentiels ont des préjugés envers les individus ayant tué un policier).

L'ordre chronologique de la requête n'est également pas associé statistiquement avec la décision des jurys, ce qui est également le cas pour la présence d'opposants durant les requêtes. À première vue, cette dernière remarque signifierait que la décision des jurys ne pourrait être associée avec la présence d'opposants lors des requêtes. Cependant, le faible nombre de requêtes incluses dans cette analyse $(n=47)$ limite la force statistique d'une association éventuelle entre ces deux variables. Il faudrait répertorier un plus grand nombre de requêtes pour vérifier l'existence d'une telle association car malgré le faible nombre de requêtes présentes, 50\% des requêtes ( 5 sur 
10) impliquant des opposants n'ont pas été réduites, contre $24 \%$ des requêtes (ou 9 sur 37) n'impliquant pas d'opposants.

\section{5 - Conclusion}

L'étendue de la couverture journalistique des requêtes de révision judiciaire varie grandement selon les différentes régions du Canada. Dans les Prairies, le pourcentage de requêtes publiées dans les journaux échantillonnés s'élève à 90\%, alors qu'il n'est que de 23\% pour le Québec. Cette différence semble attribuable au nombre de requêtes ayant eu lieu dans chaque région: plus il y a eu de requêtes, plus le critère de "nouveauté" s'estompe et conséquemment, moins les requêtes deviennent dignes d'êtres mentionnées dans les journaux. Le Québec se distingue également des autres régions par le peu d'articles consacrés à chaque requête (médiane $=1.5$ article $/ 3$ quotidiens) comparativement à d'autres régions comme les Prairies (médiane $=5.5$ articles $/ 5$ quotidiens). D'autre part, il semble que ce soit le critère de nouveauté qui explique l'évolution chronologique de la publication des requêtes car généralement, plus les années avancent, moins grande est la proportion de requêtes publiées.

La source dominante des articles diffère également selon les régions où les requêtes se sont déroulées. Au Québec et en Ontario, par exemple, le requérant est plus souvent cité ou mentionné comme source dominante qu'en Colombie-Britannique ou que dans les Prairies. En Colombie-Britannique, par contre, les proches de victime constituent une source dominante importante, alors que ce type de source dominante n'apparaît aucune fois au Québec.

L'orientation des titres et des articles, de leur côté, varient clairement de région en région. En partant de la Colombie-Britannique jusqu'au Québec, en effet, la proportion de titres et d'articles défavorables au requérant et/ou à la réduction du délai d'inadmissibilité à la libération conditionnelle diminue, alors que la proportion de titres 
et d'articles favorables augmente. D'autre part, les quotidiens tabloïds ont publié une plus grande proportion d'articles défavorables, ainsi qu'une plus petite proportion d'articles favorables, que les quotidiens de type grand format. Au niveau des récits secondaires, la proportion d'articles favorables augmente en passant des lettres d'opinion aux chroniques, et des chroniques aux éditoriaux. Finalement, l'orientation des titres et des articles varie selon le nombre de victimes tuées par le requérant: les requérants ayant tué plusieurs victimes font plus souvent l'objet d'une publicité négative que ceux n'en ayant tué qu'une. Il faut toutefois préciser que la majorité des titres et des articles concernant les meurtriers multiples référaient principalement à Clifford Olson.

Fait étonnant, la très grande majorité des articles ont rapporté des propos exacts au sujet de la nature et du fonctionnement de la révision judiciaire. Il n'y a qu'en Colombie-Britannique où l'on retrouve un peu plus d'articles inexacts qu'ailleurs au Canada. Puis, contrairement à ce qui fut postulé, les journaux tabloïds ont publié une plus grande proportion d'articles exacts que les quotidiens de type grand format.

En ce qui a trait à l'intensité de publication des requêtes, il s'avère que les requêtes impliquant un requérant ayant tué un policier font l'objet d'une couverture journalistique plus intense que les requêtes où ce n'est pas le cas. Aussi, les requêtes ayant mobilisé des opposants à la révision judiciaire sont publiées de façon plus intense que les requêtes où il n'y a pas eu d'opposants.

Finalement, la décision du jury de réduire ou non le délai d'inadmissibilité à la libération conditionnelle du requérant est associée de façon significative avec le fait que la requête ait paru ou non dans les quotidiens, ainsi qu'avec le type d'homicide commis par le requérant. Plus précisément, les requêtes ayant paru dans les journaux ont moins tendance à faire l'objet d'une réduction de délai, alors que les requérants ayant commis un homicide familial/passionnel ou un homicide associé à un autre crime ont moins de 
chance de se voir réduire leur délai comparativement à ceux ayant perpétré un homicide querelleur. 
CONCLUSION ET DISCUSSION 
Dès les premiers jours où la couverture journalistique de la justice criminelle fut scrutée par les chercheurs, un constat redondant est apparu: les journaux traitent majoritairement des crimes sensationnels et violents, l'homicide étant le crime de prédilection à couvrir (Davis, 1952; Antunes et Hurley, 1977; Graber, 1979; Ditton et Duffy, 1983; Doob, 1985; Gabor et Weimann 1987; Schlesinger et al., 1991; Jerin et Fields, 1994; Chermak, 1998a). Ainsi, la réalité telle que présentée dans les statistiques policières ne correspond pas à celle que l'on retrouve dans les journaux. Dans le cadre de la présente recherche, nous avons également découvert que la réalité concernant les requêtes de révision judiciaire, telle que présentée dans la liste fournie par le Service Correctionnel du Canada, ne concordait pas avec celle qui fut retrouvée dans les quotidiens échantillonnés.

Le premier objectif de la recherche étant d'évaluer l'étendue de la couverture journalistique des requêtes de révision judiciaire, nous avons tout d'abord constaté que le pourcentage de requêtes publiées, ainsi que l'intensité de publication de ces requêtes, ne sont pas uniformes à travers les différentes régions du Canada. Ainsi, il n'y a pas de concordance entre le nombre de requêtes ayant eu lieu dans chaque région et le nombre de requêtes publiées dans celles-ci. Au contraire, il semble que plus le nombre de requêtes s'étant déroulées dans une région augmente, moins il y a de chances que les quotidiens échantillonnés les rapportent. Il y a donc une discordance entre la réalité présentée par la liste des requêtes du SCC et celle présentée par la couverture journalistique des requêtes.

Dans la partie Ouest du Canada (Colombie-Britannique et les Prairies), les requêtes ont presque toutes paru dans les journaux, alors que les quotidiens du Québec n'en ont rapporté qu'une partie modeste. De plus, les quotidiens des régions de l'Ouest ont attribué une couverture plus intense à leurs requêtes. Donc, malgré le nombre important de requêtes qui se sont déroulées au Québec, les lecteurs des quotidiens de l'Ouest ont été plus exposés aux requêtes que l'ont été les lecteurs du Québec. Il reste 
maintenant à savoir si avec le temps, et comme ce fut probablement le cas pour le Québec, les prochaines requêtes de l'Ouest deviendront de moins en moins dignes d'être mentionnées dans les journaux étant donné leur plus grand nombre.

Nous sommes d'avis que graduellement, ces requêtes feront moins souvent l'objet d'une couverture journalistique. Cependant, cette perte d'intérêt de la part des quotidiens ne s'effectuera pas de façon aussi nette comme ce fut le cas au Québec. La requête de Clifford Olson, qui s'est déroulée en 1997 en Colombie-Britannique, a fait l'objet d'une couverture journalistique très importante et a ravivé le débat au sujet de la révision judiciaire. Conséquemment, il se pourrait que les journalistes de cette province, voire de la région de l'Ouest canadien, portent un intérêt particulier aux requêtes subséquentes puisque "The particular event is more newsworthy if it is continuous with previous events, in the sense that the reporter is able to place it within a salient frame" (Ericson et al., 1987: 143-4). Les quotidiens de cette région associant les prochaines requêtes avec celle de Clifford Olson, ils seront sûrement portés à les rapporter, elles aussi, pour un certain temps. Cette hypothèse n'a pu être vérifiée à partir de nos données car d'après la liste fournie par le SCC, la requête de Clifford Olson fut l'une des dernières à avoir eu lieu dans l'Ouest canadien.

Nous avons également constaté, à partir des articles de quotidiens, que les opposants aux requêtes de révision judiciaire se retrouvaient presque uniquement dans l'Ouest du Canada. Bien que ce constat ne fut pas présenté sous forme de tableau dans le chapitre d'analyse, il reste néanmoins que la concentration des opposants dans l'Ouest puisse influencer l'étendue de la couverture journalistique dans cette région. Tremblay (1988) avance que:

"La sensibilité de l'opinion publique à certaines questions fait en sorte qu'on parlera davantage de certains types de procès [dans les journaux]. ... Cette sensibilité peut avoir plusieurs causes. Elle peut résulter ... d'une campagne orchestrée et conduite par les forces 
policières, ... d'un mouvement social pour la défense de certains groupes sociaux." (12-13)

Dans les Prairies et en Colombie-Britannique, incidemment, les groupes s'opposant aux requêtes de révision judiciaire étaient majoritairement constitués de policiers ou de membres de groupes de pression. Or, il est fort probable qu'à certains moments, les policiers ou les membres des groupes de pression aient signalé aux maisons de presse qu'une requête de révision judiciaire allait bientôt avoir lieu à tel palais de Justice, et qu'ils s’y présenteraient pour contester cette disposition du Code criminel. Advenant que les reporters se soient également présentés, les groupes s'opposant à la révision judiciaire ont pu faire valoir leur point de vue en se faisant citer dans les journaux. Cette façon de fonctionner, si elle existe bel et bien, pourrait notamment expliquer le pourcentage élevé de requêtes publiées dans l'Ouest canadien. D'autre part, elle pourrait aussi renforcer l'idée voulant qu'avec le temps, les requêtes de l'Ouest soient davantage publiées que celles du Québec.

Peut-on cependant croire, comme semble l'insinuer Tremblay (1988), que l'opinion publique au sujet d'enjeux judiciaires se forme inévitablement et uniquement par l'entremise d'une campagne orchestrée par des groupes particuliers? Une telle affirmation réduit l'opinion publique à une éponge qui, pour se former une opinion sur un enjeu particulier, ne fait qu'absorber les propos des groupes dominants rapportés dans les médias. Cette critique s'apparente à celle émise par les tenants de l'approche institutionnelle au sujet du "média-centrisme" de l'approche de lidéologie dominante, où l'on prend pour acquis que l'idéologie dominante telle que présentée dans les médias est inévitablement assimilée au sein du grand public.

Parallèlement, Roberts (1994a) avance au sujet des révisions judiciaires que "public antipathy will be particularly strong in cases of first degree murder in which the victim was a police officer" (7). Ici encore, l'opinion publique est-elle trop rapidement confondue avec l'opinion partagée par les groupes s'opposant aux requêtes? Dans le 
cadre de la présente recherche, par exemple, les articles ayant trait aux requêtes impliquant le meurtre d'un policier ne faisaient pas état de commentaires négatifs provenant du grand public. Bien qu'il y ait eu des opposants à certaines de ces requêtes, ceux-ci étaient tous des policiers. Donc, à partir de la présente étude, nous ne pouvons pas soutenir l'affirmation de Roberts (1994a) puisque l'antipathie par rapport à ces types de requêtes ne provenait que d'un groupe particulier (les policiers) et non du public en général.

En réalisant le deuxième objectif de la recherche, qui consistait à analyser le contenu de la couverture journalistique des requêtes de révision judiciaire, nous avons constaté avec surprise que les propos rapportés au sujet de la nature et du fonctionnement de l'article 745.6 du Code criminel s'avèrent exacts en très grande majorité. Ce constat va à l'encontre des propos rapportés par certains acteurs du champ judiciaire, qui prétendent qu'il y a une quantité importante de faits inexacts dans les articles traitant de procès en droit criminel (Ericson et al., 1989; Lotz, 1991). Il y a donc peu de discordance entre l'explication de la disposition légale telle que présentée dans le Code criminel et celle rapportée dans les articles de quotidien.

Malgré cela, l'analyse de contenu a révélé que l'orientation des articles au sujet du requérant et/ou de la réduction du délai d'inadmissibilité à la libération conditionnelle variait selon les différentes régions du Canada. De la Colombie-Britannique jusqu'au Québec, en effet, la proportion d'articles défavorables diminuait constamment, alors que la proportion d'articles favorables augmentait. Les différences les plus frappantes se situaient entre la Colombie-Britannique et le Québec: au Québec, plus de la moitié des articles s'avéraient favorables, alors que plus de la moitié des articles s'avéraient défavorables en Colombie-Britannique. L'affirmation voulant que les requêtes de révision judiciaire fassent l'objet d'une couverture médiatique négative (Roberts, 1994a, 1994b, 1995, 1997) ne s'applique donc pas à l'ensemble du Canada. 
Toutefois, il faut noter que ces résultats sont largement tributaires d'une autre déformation de la réalité retrouvée dans les quotidiens: leur tendance à mettre l'accent sur les requêtes à caractère sensationnel. En Colombie-Britannique, la requête de Clifford Olson a fait l'objet d'une publicité sans précédent et ce, étant donné la réputation du meurtrier en série et l'atrocité des meurtres qu'il a commis. Conséquemment, cette requête compte pour la quasi-totalité des articles répertoriés pour la ColombieBritannique, et d'après ce qui fut rapporté dans le chapitre d'analyse, il n'est pas surprenant qu'une bonne quantité de ces articles aient été défavorables. Au Québec, par contre, la requête fortement publiée qui relevait du spectaculaire était celle de Réal Chartrand. En plus que la requête fut la première à avoir lieu au Canada, le requérant se distinguait par le fait qu'il avait tué un policier et qu'il avait été condamné à mort en premier lieu. Étant donné que presque tous les témoins ayant comparu appuyaient la réduction du délai d'inadmissibilité à la libération conditionnelle de Réal Chartrand, les articles consacrés à cette requête s'avéraient majoritairement favorables.

Non seulement ces deux requêtes peuvent-elles expliquer l'orientation générale des articles de leur région respective, mais elles pourraient également expliquer l'étendue de la couverture journalistique rattachée à ces régions. Comme il a été précisé antérieurement, la requête de Réal Chartrand n'a pas semblé être accompagnée d'un mouvement d'opposition. Or, puisque celle-ci constituait la première requête à avoir eu lieu au Québec, et qu'aucune réelle opposition ne se soit mobilisée pour se faire entendre, les journalistes ont pu en déduire que les requêtes subséquentes ne mériteraient pas une aussi grande attention médiatique. Le critère de nouveauté s'estompant suite aux quelques requêtes qui suivirent celle de Réal Chartrand, il en résulterait donc que très peu des autres requêtes ultérieures seraient publiées dans les quotidiens. En Colombie-Britannique, par contre, les prochaines requêtes risquent de faire l'objet d'une couverture relativement importante. Comme il a été expliqué plus haut, les reporters associeront certainement les autres requêtes à celle de Clifford Olson. Puisque cette requête fut fortement contestée et qu'elle a ravivé le débat entourant la légitimité-même 
de la révision judiciaire, et puisque les quotidiens doivent croire que l'opinion publique est maintenant sensibilisée à cette disposition légale, il ne serait pas surprenant que les requêtes suivantes soient également publiées.

Le troisième objectif de la recherche visait à identifier les facteurs associés à l'intensité de la couverture journalistique des requêtes de révision judiciaire. À ce sujet, il avait été trouvé que le fait que la victime du requérant ait été ou non un policier, ainsi que le fait qu'il y ait ou non des opposants présents lors de la requête, étaient associés statistiquement avec l'intensité de la couverture journalistique. Plus précisément, les requêtes impliquant le meurtre d'un policier, et celles où des opposants étaient présents, faisaient plus souvent l'objet d'une couverture journalistique intense. Cette dernière relation significative peut partiellement expliquer la faible intensité de couverture journalistique associée aux requêtes publiées au Québec. Dans les articles de quotidien traitant de ces requêtes, en effet, aucune présence d'opposants ne fut répertoriée.

Finalement, le dernier objectif de l'étude consistait à analyser la relation entre la couverture journalistique des requêtes de révision judiciaire et la décision des jurys de réduire le délai d'inadmissibilité à la libération conditionnelle. Le résultat le plus important fut qu'il y avait une relation statistiquement significative entre la parution des requêtes dans les quotidiens et la décision des jurys de réduire ce délai. Plus particulièrement, les requêtes qui avaient paru dans les quotidiens faisaient moins souvent l'objet d'une réduction que celles qui ne furent pas publiées. Ce résultat peut avoir des implications sur la perception du grand public au sujet de la révision judiciaire. Si les quotidiens tendent à couvrir les requérants ayant commis des homicides jugés plus graves ${ }^{17}$ et qui, conséquemment, risquent davantage de se voir refuser une réduction du délai d'inadmissibilité à la libération conditionnelle, le public

${ }^{17}$ Le tableau XIX montre notamment que sur les 47 requêtes publiées, 11 d'entre elles (23\%) représentent des cas où le requérant avait tué plus d'une victime. Cette donnée laisse entendre que les quotidiens s'attardent effectivement à couvrir les requêtes où le requérant a commis des gestes d'une plus grande gravité, bien que nous n'ayons pas les données au sujet des 45 requêtes non-publiées afin d'établir une comparaison globale. 
peut effectivement considérer que la révision judiciaire représente une disposition légale utilisée par des types de meurtriers qui ne sont pas représentatifs de ceux ayant habituellement recours à l'article 745.6 du Code criminel.

Malheureusement, et c'est la première limite du présent travail, nous n'avions pas en notre possession les données concernant les caractéristiques des homicides pour les requérants n'ayant pas paru dans les quotidiens. Cette carence limite donc la validité de l'affirmation selon laquelle les requérants ayant fait l'objet d'une couverture journalistique ne sont pas représentatifs de l'ensemble des requérants retrouvés dans la liste du SCC. Si nous avions eu ces informations, d'autre part, nous aurions pu évaluer les facteurs rattachés aux homicides qui seraient associés à la publication (oui/non) des requêtes, au lieu de se limiter aux facteurs associés à l'intensité de la couverture journalistique des requêtes une fois celles-ci publiées.

Une deuxième limite du travail découle du fait que plusieurs résultats sont largement tributaires du procédé d'échantillonnage des quotidiens. Le nombre de quotidiens répertoriés par province n'est effectivement pas uniforme, variant de un à trois quotidiens. Certains résultats de la présente recherche doivent donc tenir compte de cette considération méthodologique.

Finalement, une troisième limite, qui ouvre également une piste de recherche afin d'approfondir les connaissances au sujet de la couverture journalistique des requêtes de révision judiciaire, provient du fait qu'aucun journaliste ne fut interrogé pour valider certaines hypothèses proposées tout au long du travail. Les membres des groupes de pression, par exemple contactent-ils réellement les journalistes afin que ces derniers viennent couvrir les requêtes de révision judiciaire? Ou est-ce que les reporters arrivent aux palais de Justice et, fortuitement, s'aperçoivent qu'une requête mérite d'être mentionnée dans les quotidiens étant donné la présence d'un groupe manifestant contre celle-ci? Est-ce que les deux phénomènes se produisent? Des entrevues et des études 
ethnographiques auprès de chroniqueurs judiciaires s'avéreraient nécessaires afin de répondre à ces questions.

D'autre part, la réalisation d'entretiens ne devrait pas seulement se limiter qu'aux journalistes. Il se pourrait effectivement que les éditeurs de certaines maisons de presse ordonnent aux journalistes de favoriser un type d'événement judiciaire au détriment d'un autre. Or, les requêtes de révision judiciaire pourraient constituer l'un de ces sujets de prédilection. La réalisation d'entrevues auprès d'éditeurs pourrait donc contribuer à mieux comprendre le processus de construction de la nouvelle qui s'effectue autour des requêtes de révision judiciaire. 


\section{RÉFÉRENCES}


Antunes, G. E., \& Hurley, P. A. (1977). The Representation of Criminal Events in Houston's Two Daily Newspapers. Journalism Quarterly, 54(4), 756-760.

Bailey, F., \& Hale, D. E. (1998). Popular Culture, Crime \& Justice. Belmont, Calif.: West/Wadswort.

Barak, G. (1994). Media, Process, and the Social Construction of Crime: Studies in Newsmaking Criminology. New York: Garland.

Barlow, M. H., Barlow, D. E., \& Chiricos, T. G. (1995). Economic Conditions and Ideologies of Crime in the Media: A Content Analysis of Crime News. $\underline{\text { Crime \& }}$ Delinquency, 41(1), 3-19.

Berelson, B. (1952). Content Analysis in Communication Research. New York: Hafner.

Berger, P., \& Luckmann, T. (1986). La construction sociale de la réalité. Paris: Méridiens Klincksieck.

Brown, G. (1992). La révision judiciaire: qu'en est-il et comment touche-t-elle le secteur correctionnel fédéral? Forum, 4(2), 16-19.

Canada. (1987a). Réformer la sentence: une approche canadienne. Rapport de la Comission canadienne sur la détermination de la peine. Ottawa: Approvisionnements et Services Canada.

Canada. (1987b). L'accès du public et des médias au processus pénal. Ottawa: Approvisionnements et Services Canada.

Chermak, S. M. (1994). Crime in the News Media: A Refined Understanding of How Crimes Become News. in G. Barak (Ed.), Media, Process, and the Social Construction of Crime (pp. 95-129). New York: Garland.

Chermak, S. M. (1995). Victims in the News: Crime in American News Media. Boulder, CO: Westview.

Chermak, S. M. (1998a). Predicting Crime Story Salience: The Effects of Crime, Victim, and Defendant Characteristics. Journal of Criminal Justice, 26(1), 61-70.

Chermak, S. M. (1998b). Police, Courts, and Corrections in the Media. in F. Bailey, \& D. Hale (Eds.), Popular Culture, Crime and Justice (pp. 87-99). Belmont, Calif.: West/Wadsworth.

Chibnall, S. (1977). Law-and-Order News: An Analysis of Crime Reporting in the British Press. London: Tavistock. 
Ciccheti, D. V., \& Sparrow, S. S. (1981). Developing Criteria for Establishing Interrater Reliability of Specific Items: Applications of Assessment of Adaptive Behavior. American Journal of Mental Deficiency, 86, 127-137.

Cohen, S., \& Young, J. (1981). The Manufacture of News: Deviance, Social Problems and the Mass Media. London: Constable.

Cusson, M. (1987). Pourquoi punir? Paris: Dalloz.

Cusson, M., \& Boisvert, R. (1994). Recueil de données et d'analyses sur les homicides commis à Montréal de 1954 à 1962 et de 1985 à 1989 et au Québec de 1962 à 1989. École de criminologie, Université de Montréal: Centre international de criminologie comparée.

Daly, M., \& Wilson, M. (1988). Homicide. New York: Aldine De Gruyter.

Davis, J. F. (1952). Crime News in Colorado Newspapers. The American Journal of Sociology, LVII(4), 325-330.

Ditton, J., \& Duffy, J. (1983). Bias in the Newspaper Reporting of Crime News. British Journal of Criminology, 23(2), 159-165.

Doob, A. N. (1985). The Many Realities of Crime. in A. N. Doob, \& E. L. Greenspan (Eds), Perspectives in Criminal Law: Essays in Honour of John LL. J. Edwards (pp. 61-80). Aurora: Canada Law Book.

Doob, A. N., \& Roberts, J. V. (1983). La détermination de la peine: analyse de l'opinion publique sur la détermination de la peine. Ottawa: Ministère de la Justice.

Doob, A. N., Sprott, J. B., Marinos, V., \& Varma, K. N. (1998). An Exploration of Ontario Residents' Views of Crime and the Criminal Justice System: A Report to Operation Springboard. University of Toronto: Centre of Criminology.

Drechsel, R. (1978). How Minnesota Newspapers Cover the Trial Courts. Judicature, 62(4), 195-202.

Drechsel, R. (1983). News Media in the Trial Courts. New York: Longman.

Drechsel, R., Netteburg, K., \& Aborisade, B. (1980). Community Size and Newspaper Reporting of Local Courts. Journalism Quarterly, 57(1), 71-78.

Dussuyer, I. (1979). Crime News: A Study of 40 Ontario Newspapers. Toronto: University of Toronto.

Ericson, R. V. (1995). Crime and the Media. Aldershot: Dartmouth. 
Ericson, R. V. (1991). Mass Media, Crime, Law, and Justice: An Institutional Approach. The British Journal of Criminology, 31(3), 219-249.

Ericson, R. V., Baranek, P. M., \& Chan, J. B. L. (1987). Visualizing Deviance: A Study of News Organizations. Toronto: University of Toronto Press.

Ericson, R. V., Baranek, P. M., \& Chan, J. B. L. (1989). Negotiating Control: A Study of News Sources. Toronto: University of Toronto Press.

Ericson, R. V., Baranek, P. M., \& Chan, J. B. L. (1991). Representing Order: Crime, Law, and Justice in the News Media. Toronto: University of Toronto Press.

Fishman, M. (1978). Crime Waves as Ideology. Social Problems, 25, 531-543.

Fishman, M. (1980). Manufacturing the News. Austin: University of Texas Press.

Freedman, J. L., \& Burke, T. M. (1996). The Effect of Pretrial Publicity: The Bernardo Case. Canadian Journal of Criminology, 38(3), 253-270.

Gabor, T., \& Weimann, G. (1987). La couverture du crime par la presse: un portrait fidèle ou déformé? Criminologie, XX(1), 79-98.

Gaucher, R., \& Crow, M. (1994). Judicial Review Hearings (Criminal Code s.745): A Preliminary Study of the First 40 Cases. Ottawa: Université d’Ottawa.

Gorelick, S. (1989). Join Our War: The Construction of Ideology in a Newspaper CrimeFighting Campaign. Crime and Delinquency, 35, 421-436.

Graber, D. A. (1979). Is Crime News Coverage Excessive? Journal of Communication, 29 $(3), 81-92$.

Graber, D. A. (1980). Crime News and the Public. New York: Praeger.

Grabosky, P., \& Wilson, P. (1989). Journalism \& Justice: How Crime is Reported. Sydney: Pluto.

Grandmaison, R. (1993). Les décisions pénales dans les causes d'homicide au tribunal de Montréal, de 1985 à 1989. Université de Montréal: Mémoire de maîtrise de l'École de criminologie.

Greene, E. (1990). Media Effects on Jurors. Law and Human Behavior, 14(5), 439-450.

Greene, E. G., \& Loftus, E. F. (1984). What's New in the News? The Influence of WellPublicized News Events on Psychological Research and Courtroom Trials. Basic and Applied Social Psychology, 5(3), 211-221. 
Guba, E. G., \& Lincoln, Y. S. (1994). Competing Paradigms in Qualitative Research. in N. K. Denzin, \& Y. S. Lincoln (Eds.), Handbook of Qualitative Research (pp. 105-117). Thousand Oaks: Sage.

Hall, S. (1986). Media Power and Class Power. in J. Curran, J. Ecclestone, G. Oakley, \& A. Richardson (Eds.), Bending Reality: The State of the Media (pp. 5-14). London: Pluto Press.

Hall, S., Critcher, C., Jefferson, T., Clarke, J., \& Roberts, B. (1978). Policing the Crisis: Mugging, the State, and Law and Order. New York: Holmes \& Meier.

Humphries, D. (1981). Serious Crime, News Coverage, and Ideology: A Content Analysis of Crime Coverage in a Metropolitan Paper. Crime and Delinquency, 27(2), 191-205.

Jerin, R. A., \& Fields, C. B. (1994). Murder and Mayhem in USA Today: A Quantitative Analysis of the National Reporting of States' News. in G. Barak (Ed.), Media, Process, and the Social Construction of Crime: Studies in Newsmaking Criminology (pp. 187-202). New York: Garland.

Johnson, G ., Austin, I., \& Jiwa, S. (August 22, 1997). Unreliable liar's claims draw judge's fire. The Province (Vancouver), A3.

Johnstone, J. W. C., Hawkins, D. F., \& Michener, A. (1994). Homicide Reporting in Chicago Dailies. Journalism Quarterly, 71 (4), 860-872.

Kelly, M. (1986). L'analyse de contenu. in B. Gauthier ((Dir.)), Recherche sociale: de la problématique à la collecte des données (pp. 293-315). Québec: Presses de l'Université du Québec.

Kent, T. (1985). Media Influence on Opinion and Policy. in D. Gibson, \& J. K. Baldwin (Eds.), Law in a Cynical Society? Opinion and Law in the 1980's (pp. 341-349). Calgary: Carswell.

Kerr, N. L. (1994). The Effects of Pretrial Publicity on Jurors. Judicature, 78(3), 120127.

Kramer, G. P., Kerr, N. L., \& Carroll, J. S. (1990). Pretrial Publicity, Judicial Remedies, and Jury Bias. Law and Human Behavior, 14(5), 409-438.

Lotz, R. E. (1991). Crime and the American Press. New York: Praeger.

Lundsgaarde, H. P. (1977). Murder in Space City: A Cultural Analysis of Houston Homicide Patterns. New York: Oxford University Press. 
Marquez, F. T. (1980). How Accurate Are the Headlines? Journal of Communication, 30(3), 30-36.

Martel, J. (1994). Femme battue et mari "batteur": une reconstruction médiatique dans La Presse au XXIXe siècle. Criminologie, XXVII(1), 117-134.

McCormick, C. (1995). Constructing Danger: The Mis/representation of Crime in the News. Halifax: Fernwood.

Morissette, R. (1991). La presse et les tribunaux: un mariage de raison. Montréal: Quebecor.

Ogloff, J. R. P., \& Vidmar, N. (1994). The Impact of Pretrial Publicity on Jurors: A Study to Compare the Relative Effects of Television and Print Media in a Child Sex Abuse Case. Law and Human Behavior, 18(5), 507-525.

O'Reilly-Fleming. (1991). The Injustice of Judicial Review: Vaillancourt Reconsidered. Canadian Journal of Criminology, 33(2), 163-170.

Parent, G.-A. (1987). Presse et corps policiers: complicité et conflit. Criminologie, $\underline{\mathrm{XX}}(1), 99-120$.

Pilon, M. (1997). Murderers' Parole Eligibility: The Evolution of Judicial Review. Canada: Library of Parliament.

Poirier, R. (1987). La négociation des sentences du point de vue des avocats de la défense. Criminologie, XX(2), 57-68.

Pritchard, D. (1986). Homicide and Bargained Justice: The Agenda-Setting Effect of Crime News on Prosecutors. Public Opinion Quarterly, 50, 143-159.

Pritchard, D., \& Hughes, K. D. (1997). Patterns of Deviance in Crime News. Journal of Communication, 47(3), 49-67.

Roberts, J. V. (1988a). La détermination de la peine dans les médias: Une analyse du contenu des journaux de langue anglaise au Canada. Rapports de recherche de la Commission canadienne sur la détermination de la peine. Ottawa: Ministère de la Justice du Canada.

Roberts, J. V. (1988b). L'opinion publique et la détermination de la sentence: les sondages de la commission canadienne sur la détermination de la peine. Rapports de recherche de la Commission canadienne sur la détermination de la peine. Ottawa: Ministère de la Justice du Canada. 
Roberts, J. V. (1992). Public Opinion, Crime, and Criminal Justice. in M. Tonry (Ed.), Crime and Justice: A Review of Research (Vol. 16pp. 99-180). Chicago: The University of Chicago Press.

Roberts, J. V. (1994a). Judicial Review of Parole Eligibility: The Views of the Public: "Life-line or Loophole?". University of Ottawa, Ottawa: Department of Criminology. Version préliminaire.

Roberts, J. V. (1994b). Public Knowledge of Crime and Justice: An Inventory of Canadian Findings. Technical Report. Ottawa: Department of Justice Canada.

Roberts, J. (1995). La libération conditionnelle pour les condamnés à perpétuité: une décision qui revient à des citoyens. Actualités-Justice, 11(2), 15-16.

Roberts, J. (1997). L'article 745: une question de compromis. Actualités-Justice, 12(2), 4-5.

Roberts, J. V., \& Doob, A. N. (1990). News Media Influences on Public Views of Sentencing. Law and Human Behavior, 14(5), 451-468.

Rosenfeld, E. (1988). Méthodes, politiques et subjectivité: Étude sur la politique rédactionnelle de certains médias en matière de détermination de la peine. Rapport de recherche de la Commission canadienne sur la détermination de la peine. Ottawa: Ministère de la Justice du Canada.

Schlesinger, P. (1989a). Rethinking the Sociology of Journalism: Source Strategies and the Limits of Media Centrism. in M. Ferguson (Ed.), Public Communication: The New Imperatives . London: Sage.

Schlesinger, P. (1989b). From Production to Propaganda? Media, Culture and Society, 11, 283-306.

Schlesinger, P., \& Tumber, H. (1994). Reporting Crime: The Media Politics of Criminal Justice. New York: Oxford University Press.

Schlesinger, P., Tumber, H., \& Murdock, G. (1991). The Media Politics of Crime and Criminal Justice. British Journal of Sociology, 42(3), 397-420.

Soothill, K., \& Walby, S. (1991). Sex Crime in the News. London: Routledge.

Stanga, J. E. Jr. (1971). The Press and the Criminal Defendant: Newsmen and Criminal Justice in Three Wisconsin Cities. University of Wisconsin: University Microfilms.

Statistique Canada. (1997). Le Canada, sa culture, son patrimoine et son identité: Perspective statistique. Ottawa: Ministre de l'Industrie. 
Surette, R. (1984). Justice and the Media: Issues and Research. Springfield, Ill.: Charles C. Thomas.

Surette, R. (1992). Media, Crime, and Criminal Justice: Images and Realities. Pacific Grove, Calif.: Brooks/Cole.

Surette, R. (1998). Media, Crime, and Criminal Justice: Images and Realities. 2nd Ed. Belmont, Calif.: West/Wadsworth.

Tremblay, G. (1988). Recherche sur les stratégies et pratiques des médias en matière d'information judiciaire. Rapport de recherche de la Commission canadienne sur la détermination de la peine. Ottawa: Ministère de la Justice du Canada.

Voumvakis, S. E., \& Ericson, R. V. (1984). News Accounts of Attacks on Women: A Comparison of Three Toronto Newspapers. Toronto: University of Toronto.

Websdale, N., \& Alvarez, A. (1998). Forensic Journalism as Patriarchal Ideology: The Newspaper Construction of Homicide-Suicide. in F. Bailey, \& D. Hale (Eds.), Popular Culture, Crime, and Justice (pp. 123-141). Belmont, Calif.: West/Wadsworth.

Welch, M., Fenwick, M., \& Roberts, M. (1997). Primary Definitions of Crime and Moral Panic: A Content Analysis of Experts' Quotes in Feature Newspaper Articles on Crime. Journal of Research in Crime and Delinquency, 34(4), 474494.

Wilbanks, W. (1984). Murder in Miami: An Analysis of Homicide Patterns and Trends in Dade County, Florida, 1917-1983. New York: University Press of America.

Williams, P., \& Dickinson, J. (1993). Fear of Crime: Read All about it? The Relationship between Newspaper Crime Reporting and Fear of Crime . British Journal of Criminology, 33(1), 33-56. 
ANNEXE A 
La variable "orientation de l'article" vise à évaluer l'orientation de l'article (favorable, défavorable, équilibré ou neutre) au sujet du requérant et/ou de la réduction du délai d'inadmissibilité à la libération conditionnelle. À noter que lors de la codification des articles, la description du meurtre commis par le requérant était considérée comme étant neutre sauf si des qualificatifs tels "meurtre sordide" ou "horrible meurtre" avaient été insérés. Dans ces derniers cas, la description du meurtre était considérée comme un élément défavorable au requérant.

Voici donc la description des quatre catégories de la variable:

Favorable: En général, le contenu manifeste de l'article est favorable au requérant et/ou à la réduction du délai d'inadmissibilité à la libération conditionnelle.

exemples: - l'article traite principalement d'un psychologue venu témoigner pour dire que le requérant exprime des remords face au crime qu'il a commis et qu'il est maintenant prêt à réintégrer la société vu son comportement exemplaire dans les pénitenciers.

- suite à la décision du jury de considérer une réduction du délai d'inadmissibilité à la libération conditionnelle, l'article traite principalement du témoignage des proches du requérant. Ceux-ci font part de leur joie étant donné la nature de la décision.

Défavorable: En général, le contenu manifeste de l'article est défavorable au requérant et/ou à la réduction du délai d'inadmissibilité à la libération conditionnelle.

exemples: - l'article traite principalement des propos rapportés par le procureur de la Couronne lors des audiences. Durant celles-ci, le procureur de la Couronne a affirmé que le requérant représentait toujours un danger pour la société, et qu'il a joué le jeu en 
s'inscrivant à des programmes de réhabilitation uniquement au cours des trois dernières années précédant la requête.

- suite à une décision du jury de ne pas considérer une réduction du délai d'inadmissibilité à la libération conditionnelle, les proches de la victime tuée par le requérant expriment leur mécontentement face au mécanisme de révision judiciaire. Ils affirment que ce mécanisme ne constitue qu'une perte de temps et d'argent, et que la requête à laquelle ils viennent d'assister n'a fait que ramener de tristes souvenirs au sujet du décès de leur proche.

Équilibré: En général, le contenu manifeste de l'article est aussi bien favorable que défavorable au sujet du requérant et/ou de la réduction du délai d'inadmissibilité à la libération conditionnelle.

exemple: - durant les audiences, un agent correctionnel témoigne en faveur du requérant en faisant état de son comportement exemplaire dans les pénitenciers, alors qu'un psychologue témoigne en défaveur en rapportant que le requérant n'exprime aucun remords face à l'homicide qu'il a commis.

Neutre: En général, le contenu manifeste de l'article est neutre au sujet du requérant et/ou de la réduction du délai d'inadmissibilité à la libération conditionnelle.

exemple: - un article traite brièvement de la requête en ne faisant que résumer la décision rendue par le jury. 
La variable "orientation du titre de l'article" vise à évaluer l'orientation du titre de l'article (favorable, défavorable, équilibré ou neutre) au sujet du requérant et/ou de la réduction du délai d'inadmissibilité à la libération conditionnelle.

Voici donc la description des quatre catégories de la variable:

Favorable: En général, le contenu manifeste du titre de l'article est favorable au requérant et/ou à la réduction du délai d'inadmissibilité à la libération conditionnelle.

exemple: - le titre de l'article écrit: "Le requérant s'est comporté de façon exemplaire dans les pénitentiers selon les dires d'un agent correctionnel".

Défavorable: En général, le contenu manifeste du titre de l'article est défavorable au requérant et/ou à la réduction du délai d'inadmissibilité à la libération conditionnelle.

exemple: - le titre de l'article écrit: "'Le requérant n'est pas réhabilité", proclame le procureur de la Couronne".

Équilibré: En général, le contenu manifeste du titre de l'article est aussi bien favorable que défavorable au sujet du requérant et/ou de la réduction du délai d'inadmissibilité à la libération conditionnelle.

exemple: - le titre de l'article écrit: ""Il n'est pas prêt à sortir" dit le procureur de la Couronne; "Il a suffisamment payé" selon l'avocate de la défense.

Neutre: En général, le contenu manifeste du titre de l'article est neutre au sujet du requérant et/ou de la réduction du délai d'inadmissibilité à la libération conditionnelle.

exemple: - le titre de l'article écrit: "Requête de Gagnon: le jury délibère". 
ANNEXE B 
Tableau B1:

Exactitude de l'article selon le type de récit secondaire

\begin{tabular}{|c|c|c|c|}
\hline $\begin{array}{l}\text { Type de récit } \\
\text { Secondaire }\end{array}$ & Exact (\% rangée) & $\begin{array}{l}\text { Exactitude de l'article } \\
\text { Inexact (\% rangée) }\end{array}$ & Total (\% rangée) \\
\hline Dossier & $4(80 \%)$ & $1(20 \%)$ & $5(100 \%)$ \\
\hline Lettre d'opinion & $5(83 \%)$ & $1(17 \%)$ & $6(100 \%)$ \\
\hline Éditorial & 5 (83\%) & $1(17 \%)$ & $6(100 \%)$ \\
\hline Chronique & 11 (85\%) & $2(15 \%)$ & $13(100 \%)$ \\
\hline Total & 25 (83\%) & $5(17 \%)$ & 30 (100\%) \\
\hline
\end{tabular}

Tableau B2:

Exactitude de l'article selon les caractéristiques liées aux homicides

\begin{tabular}{cccc}
\hline Type de & Exact (\% rangée) & $\begin{array}{c}\text { Exactitude de l'article } \\
\text { Inexact (\% rangée) }\end{array}$ & Total (\% rangée) \\
Condamnation & $194(89 \%)$ & $23(11 \%)$ & $217(100 \%)$ \\
$1^{\text {er }}$ degré & $14(93 \%)$ & $1(7 \%)$ & $15(100 \%)$ \\
2e degré & $13(93 \%)$ & $1(7 \%)$ & $14(100 \%)$ \\
Capital & $13(81 \%)$ & $3(19 \%)$ & $16(100 \%)$ \\
Non-capital & $234(89 \%)$ & $28(11 \%)$ & $262(100 \%)$ \\
Total & & & \\
Sexe de la victime & $184(89 \%)$ & $23(11 \%)$ & $207(100 \%)$ \\
\hline Homme & $50(91 \%)$ & $5(9 \%)$ & $55(100 \%)$ \\
Femme & $234(89 \%)$ & $28(11 \%)$ & $262(100 \%)$ \\
Total & & & \\
Type de victime & $91(91 \%)$ & $9(9 \%)$ & $100(100 \%)$ \\
Adulte & $15(94 \%)$ & $16 \%)$ & $16(100 \%)$ \\
Jeune & $125(87 \%)$ & $18(13 \%)$ & $143(100 \%)$ \\
Policier & $231(89 \%)$ & $28(11 \%)$ & $259(100 \%)$ \\
Total & & & \\
Type d'homicide & $62(92 \%)$ & $5(8 \%)$ & $67(100 \%)$ \\
\hline Querelleur & $18(86 \%)$ & $3(14 \%)$ & $21(100 \%)$ \\
Familial ou passionnel & $141(88 \%)$ & $19(12 \%)$ & $160(100 \%)$ \\
Associé à un crime & $221(89 \%)$ & $27(11 \%)$ & $248(100 \%)$ \\
Total & & & \\
\hline Nombre de victimes & $234(89 \%)$ & $28(11 \%)$ & $262(100 \%)$ \\
\hline Une & $67(83 \%)$ & $14(17 \%)$ & $81(100 \%)$ \\
Deux et plus & $301(88 \%)$ & $42(12 \%)$ & $343(100 \%)$ \\
Total & & &
\end{tabular}

